\title{
Nothing to lose? : cognitive and behavioral therapy for obesity and binge eating disorder
}

Citation for published version (APA):

Nauta, H. M. (2001). Nothing to lose? : cognitive and behavioral therapy for obesity and binge eating disorder. [Doctoral Thesis, Maastricht University]. Universiteit Maastricht. https://doi.org/10.26481/dis.20011108hn

Document status and date:

Published: 01/01/2001

DOI:

10.26481/dis.20011108hn

Document Version:

Publisher's PDF, also known as Version of record

\section{Please check the document version of this publication:}

- A submitted manuscript is the version of the article upon submission and before peer-review. There can be important differences between the submitted version and the official published version of record.

People interested in the research are advised to contact the author for the final version of the publication, or visit the DOI to the publisher's website.

- The final author version and the galley proof are versions of the publication after peer review.

- The final published version features the final layout of the paper including the volume, issue and page numbers.

Link to publication

\footnotetext{
General rights rights.

- You may freely distribute the URL identifying the publication in the public portal. please follow below link for the End User Agreement:

www.umlib.nl/taverne-license

Take down policy

If you believe that this document breaches copyright please contact us at:

repository@maastrichtuniversity.nl

providing details and we will investigate your claim.
}

Copyright and moral rights for the publications made accessible in the public portal are retained by the authors and/or other copyright owners and it is a condition of accessing publications that users recognise and abide by the legal requirements associated with these

- Users may download and print one copy of any publication from the public portal for the purpose of private study or research.

- You may not further distribute the material or use it for any profit-making activity or commercial gain

If the publication is distributed under the terms of Article $25 \mathrm{fa}$ of the Dutch Copyright Act, indicated by the "Taverne" license above, 


\section{NOTHING TO LOSE?}

COGNITIVE AND BEHAVIORAL THERAPY

FOR OBESITY AND BINGE EATING DISORDER 


\title{
\$32O1 OT DИIHTOV
}

\author{
YQAЯ3HT JAMOIVAHGS OИA JVITIИDOS

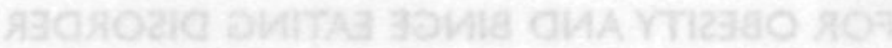




\title{
NOTHING TO LOSE?
}

\author{
COGNITIVE AND BEHAVIORAL THERAPY \\ FOR OBESITY AND BINGE EATING DISORDER
}

\section{PROEFSCHRIFT}

ter verkrijging van de graad van doctor

aan de Universiteit Maastricht,

op gezag van de Rector Magnificus,

Prof. dr.A.C. Nieuwenhuijzen Kruseman

volgens het besluit van het College van Decanen,

in het openbaar te verdedigen op

donderdag 8 november 2001 om 14.00 uur

door

Helga Mariëtte Nauta 


\title{
\$20」 OT DИHHTOK
}

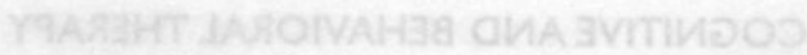 \\ я3СЯ
}

T719HO273098

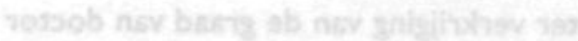

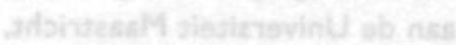

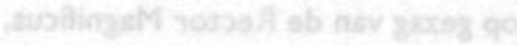

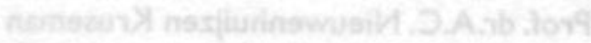

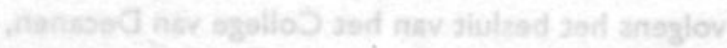
qo neglbabrov ay zenchaço red a

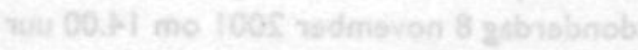

noob

aryoh wathat4 solot 


\section{VENUS OF WILLENDORF}

By Yusef Komunyakaa

She's big as a man's fist,

Big as a black-pepper shaker

Filled with gris-gris dust,

Like two fat gladiolus bulbs

Grown into a burst of twilight.

Lumpy \& fertile, earthy

\& egg-shaped, she's pregnant

With all the bloomy hosannas

Of love-hunger. Beautiful

In a way that forces us to look

At the ground, this squat

Venus in her braided helmet

Is carved from a hunk of limestone

Shaped into a blues singer.

In her big smallness

She makes us kneel. 


\section{Promotor}

Prof. dr.A.T.M. Jansen

Co-promotor

Dr. H.J. Hospers

\section{Beoordelingscommissie}

Prof. dr. ir. J. Brug (voorzitter)

Dr. B.J.F. Boon

Prof dr. P.M.G. Emmelkamp (Universiteit van Amsterdam)

Prof. dr. M.A. van den Hout

Dr. F.L.Wojciechowski

\section{Colofon}

Lay-out: Nora Oosting, Maastricht

Ontwerp omslag: Josee Driessen, Amsterdam

Druk: Unigraphic, Maastricht

ISBN 90-5681-116-9

The studies presented in this thesis were performed at the Maastricht Health Research Institute for Prevention and Care (HEALTH), which participates in the Netherlands School of Primary Care Research ( $\mathrm{CaRe}$ ), acknowledged in 1995 by the Royal Dutch Academy of Sciences (KNAW). 


\section{CONTENTS}

Chapter I: Obesity and binge eating disorder

Chapter 2: An cognitive-behavioral model for the treatment of obesity and binge eating disorder

Chapter 3 Treatment protocols and outline of this thesis

Chapter 4 Cognitions in obese binge eaters and obese non-binge eaters

Chapter 5 A comparison between a cognitive and a behavioral therapy for obese binge eaters and obese non-binge eaters

Chapter 6 One-year follow-up effects of two obesity treatments on psychological well-being and weight

Chapter 7 Pretreatment patient characteristics as predictors of one-year outcome after treatment for obesity and the influence of shape and weight concerns

Chapter 8 Main conclusions and general discussion

References

Summary

Samenvatting

Dankwoord

Curriculum Vitae 


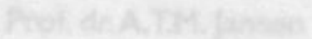

e

Co protunior

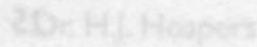

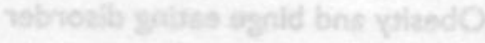

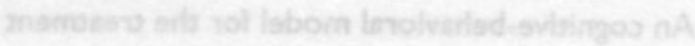

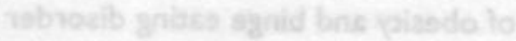

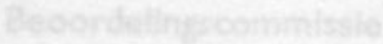

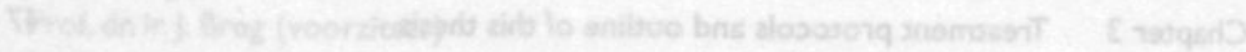

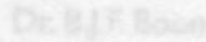

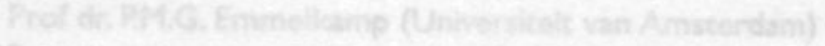

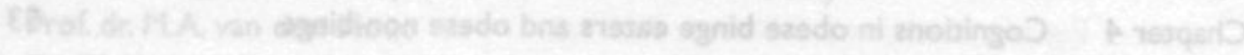

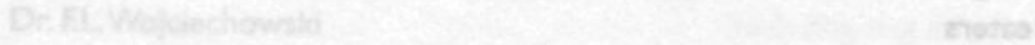

fad

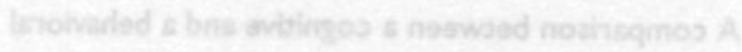

2 masando

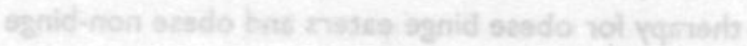

20108

8

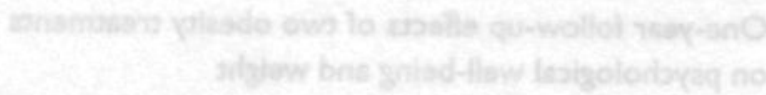

dovesaerto

Pe

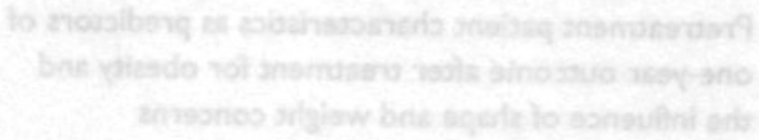

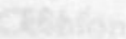

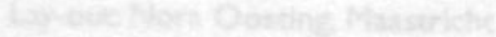

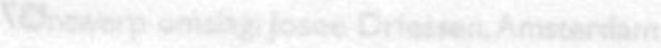

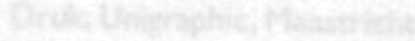

IAY 


\section{OBESITY AND BINGE EATING DISORDER}

\section{INTRODUCTION}

This dissertation focuses on women with obesity and binge eating disorder and the effectiveness of a non-dieting behavioral and a cognitive therapy for both groups. In Western culture, being overweight is generally viewed as a more serious problem for females than for males (Harris, Walters, \& Waschull, 1991). Females worry more about their shape and weight, are more critical about their bodies, and want to weigh less (e.g., Brown \& Bentley-Condit, 1998; Pingitore, Spring, \& Garfield, 1997; Striegel-Moore, Silberstein, \& Rodin, 1986). The desire for thinness has led to an increase of weight loss programs, ma-gazine articles and books about the newest and quickest ways to lose weight.

The first Chapter gives a brief historical framework for the development of ideas about obesity, as well as a description of the definition and prevalence of obesity. A subgroup of obese persons reporting problems with binge eating is introduced and the differences in eating disorder symptomatology and comorbidity between obese binge eaters and obese non-binge eaters is given. The present weight loss treatments for obese persons will be critically reviewed. 
The second Chapter introduces a non-dieting cognitive-behavioral model for obese binge eaters and obese non-binge eaters. This model is based on theories and ideas about eating behavior and dysfunctional cognitions in obese binge eaters and obese non-binge eaters. An update is given of cognitive-behavioral therapy for obese binge eaters and obese non-binge eaters.

The third Chapter outlines the treatment protocols and introduces the empirical studies of this thesis.

\section{HISTORICAL FRAMEWORK FOR THE DEVELOPMENT OF IDEAS ABOUT OBESITY}

Historical references showed that ideas about obesity have changed through the years. In prehistory, a positive connection was drawn between excess weight and fertility. The famous Venus of Willendorf for example, a small 25.000year-old limestone statue of a very heavy female figure, was thought to be a fertility symbol (Bray, 1990). This view changed later in a more negative way. The ancient Greeks disliked fat people. The Greeks suggested that obese persons are more sensible to sudden death, infertility and irregular menses. According to Hippocrates remedies for obesity included; eating food products high in fat, eating once a day, sleeping on a hard bed, and walking naked as long as possible (Brownell, 1995). The 18th and 19th centuries showed a shift to a moral approach, which saw personal shortcomings - i.e. excessive eating and lack of exercise - as the cause of obesity. A vast amount of research on obesity was conducted in the 20th century. Until 1950, the field of obesity was dominated by medical and biological science. The negative consequences of obesity were

10 generally defined in medical terms only, while other important aspects of the obese, such as psychological or social components, were ignored (Jansen, 1998). Although it still is the ultimate goal to lose weight, the means used to accomplish this goal have varied to a great extent. In the 1950 s, the discipline of psychiatry, dominated by psychoanalytic theory, joined medicine in the treatment of obesity. At that time, obesity was characterized by psychoanalysts as a symptom of underlying emotional disturbances arising from unsatisfactory experiences during infancy. Psychologists entered the picture in the 1960 s and 1970 s with the application of learning theory. Initially, the basic assumption behind behavioral therapy was that obesity was simply due to excessive food intake. However, behavioral programs for obese people focusing on weight loss lead to short-term success, but are often not very effective in maintaining longterm weight loss. In the 1990 s pessimism about the ability of obese persons to lose weight permanently even increased. This led to a strong anti-dieting movement (Wilson, 1995). More recent, studies have led to some degree of optimism that better treatment outcome will result from matching specific treat- 
ments to particular subgroups (Brownell \& Wadden, 1991) and in the application of psychotherapeutic methods such as cognitive therapy (Brownell \& Wadden, 1992).

\section{DEFINITIONAND PREVALENCE OF OBESITY}

Obesity and being overweight are the most commonly used words for increased body fat and have replaced such older terms as corpulence, adiposity and polysarcie (Bray, 1990). One of the most widely used ways of assessing overweight is the Body Mass Index (BMI) (Brownell, 1995). The BMI is expressed as weight in kilograms $(\mathrm{kg}$ ) divided by length in meters squared. Presently, in the United States there seems to be a preference for the BMI figures adopted by the National Center for Health Statistics, which in the past 20-years performed five surveys among a representative sample of Americans in the United States (Kuczmarski, Flegal, Campbell, \& Johnson, 1994). The latter define overweight as weights exceeding the 85 th percentile of weight for height, using the reference values of 20-29 year olds, and severe overweight or obesity as the 95th percentile of weight for height among 20-29 year olds in the same sample. Using this technique they define overweight as a BMI exceeding 27.3 for women and above 27.8 for men, and severe overweight or obesity as a BMI exceeding 32.3 for women and above 31.1 for men. The World Health Organization (WHO) has recently proposed a classification based on four degrees of overweight (WHO, 1995). The WHO defined a BMI between 25 and 30 as pre-obese, a BMI between 30 and 35 as obesity class I, a BMI between 35 and 40 as obesity class II, and finally a BMI above 40 as obesity class III. In this thesis, a BMI above 27 is referred to as overweight and a BMI above 30 is referred to as obese.

Obesity is a prevalent and serious problem. Worldwide, the highest reported prevalence of obesity is among Pacific Islanders. In Nauru (Micronesia) the prevalence of obesity is $65 \%$ in males and $70 \%$ in females (Seidell \& Rissanen, 1998). In the United States $20 \%$ of the males and $25 \%$ of the females can be considered obese (WHO, 1997). Obesity is also a widespread problem in Europe, particularly in women from southern and eastern parts of Europe (Seidell, 1995). In Europe, there is also a higher prevalence of obesity in rural than in urban areas (Kluthe \& Schubert, 1985). Furthermore, socioeconomic class is a powerful predictor of the prevalence of obesity. In Europe, obesity is more prevalent in the lower socioeconomic groups (Sobal \& Stunkard, 1989). Seidell and Rissanen (1998) give an overall prevalence estimate for obesity in Europe in the range of $10 \%$ to $20 \%$ in men and $15 \%$ to $25 \%$ in women.

Between 1989 and 2000 , the prevalence of obesity $(B M I>30)$ in the Netherlands increased from $5.0 \%$ to $8.6 \%$ in males and from $6.3 \%$ to $10.2 \%$ in 
females. During the same period, the prevalence of overweight (BMI>27) in the Netherlands increased from $19.7 \%$ to $26.0 \%$ in males and from $18.6 \%$ to $24.0 \%$ in females (Website Centraal Bureau voor Statistiek, 2001). Diminished physical activity, increased availability and consumption of high-fat products, and inadequate adjustments of energy intake to the diminished energy requirements are likely to be major determinants of the increased prevalence of obesity (Seidell \& Rissanen, 1998). In the Netherlands, as well as in other European countries, there is a strong inverse correlation between educational level and obesity. In $2000,17.5 \%$ of the Dutch adults with relatively low education levels were obese as compared to $5.1 \%$ of the Dutch adults with relatively higher levels of education (Website Centraal Bureau voor Statistiek, 2001). The prevalence of obesity in Dutch men increases up to the age of 55 years and then decreases. In women the prevalence of obesity increases continuously with age (Seidell, 1995).

\section{DEFINITION OF BINGE EATING DISORDER}

An important development in the study of obesity is the finding that between $20 \%$ and $46 \%$ of obese patients seeking treatment report moderate to severe problems with binge eating (Bruce \& Wilfley, 1996; Gormally, Black, Daston, \& Rardin, 1982; Marcus, Wing, \& Lamparski, 1985; Spitzer et al., 1992; Telch, Agras, \& Rossiter, 1988). This subgroup of obese persons displays even more severe psychological problems than obese non-binge eaters seeking treatment. Binge eating disorder (BED), originally described by Stunkard (1959), consists of eating large amounts of food in a short period of time. This eating is accompanied by perceived loss of control and subsequent distress. Unlike bulimia nervosa patients, binge eaters do not use any compensatory behaviors following a binge. In recent years, there has been increased interest in the exploration of binge eaters as a relatively homogenous subgroup among the obese. The diagnosis of BED has been included in the most recent version of the Diagnostic and Statistical Manual of Mental Disorders (APA, 1994) as an eating disorder Not Otherwise Specified, and as a proposed diagnostic category requiring further study. The preliminary criteria for BED are listed in Table 1.1.

Some critical remarks about the diagnostic criteria should be made. First, a diagnostic concern of the DSM-IV criteria centers on binge eating in a discrete period of time. In practice, binge eating among BED patients is difficult to diagnose, because some obese patients report overeating with loss of control that during any discrete period might not be judged as immoderate, but that over the course of the day would be considered excessive. This pattern of eating has been referred to as grazing and has been reported in several studies (Marcus, Smith, Santelli, \& Kaye, 1992; Rossiter, Agras, Telch, \& Bruce, 1992). Whether 
A Recurrent episodes of binge eating. An episode of binge eating is characterized by both of the following:

I Eating, in a discrete period of time (e.g., within any 2 hour period), an amount of food that is definitely larger than most people would eat in a similar period of time under similar circumstances

2 A sense of lack of control over eating during the episode (e.g., a feeling that one cannot stop eating or control what or how much one is eating)

B The binge eating episodes are associated with three (or more) of the following:

I Eating much more rapidly than normal

2 Eating until feeling uncomfortably full

3 Eating large amounts of food when not feeling physically hungry

4 Eating alone because of being embarrassed by how much one is eating

5 Feeling disgusted with oneself, depressed or very guilty after overeating

C Marked distress regarding binge eating is present

D The binge eating occurs, on average, at least 2 days a week for 6 months

E The binge eating is not associated with the regular use of inappropriate compensatory behaviors (e.g.. purging, fasting, excessive exercise) and does not occur exclusively during the course of anorexia nervosa or bulimia nervosa

obese binge eaters who consume large amounts of food in a short period of time differ clinically from those who eat smaller amounts over a longer period continues to be an interesting topic for further research.

Second, although weight is not mentioned for the diagnostic criteria for either BED or bulimia nervosa, weight may be relevant to diagnosis. Almost all BED patients are overweight and almost all bulimia patients are of normal weight. Normal weight binge eaters tend to show distress about their binge episodes. In contrast, obese binge eaters usually tend to display distress with the primary complaint of excessive weight. Besides, for obese binge eaters weight and shape concerns are generally more realistic than for normal weight binge eaters.

Third, loss of control and distress about overeating are not the full story about how persons with BED experiences their binge episodes. Mitchell and colleagues (1999) compared participants with BED and participants with bulimia nervosa. Both groups were identical in terms of their distress about binge eating. However, persons with BED often reported that bingeing was making them feel relaxed, whereas those suffering from bulimia reported that it was making them feel anxious. Also, persons with BED reported that they enjoyed the food, the taste and smell more often than bulimia nervosa patients. 
Binge eating disorder is the most common eating disorder, affecting about 2$5 \%$ of the community (Gotestam \& Agras, 1995; Spitzer et al., 1992; Spitzer et al., 1993). Its prevalence increases with increasing levels of obesity, affecting between $20 \%$ and $46 \%$ of obese persons seeking specialized treatment (Bruce \& Wilfley, 1996; Gormally et al., 1982; Marcus, Wing, \& Lamparski, 1985; Spitzer et al., 1992; Telch, Agras, \& Rossiter, 1988). The prevalence of binge eating among massively obese patients undergoing bariatric surgery may even exceed $50 \%$ (Adami, Gandolfo, Bauer, \& Scopinaro, 1995). On average, BED is diagnosed in persons who are in their mid- to late thirties (Yanovski, 1993). However, onset of binge eating is often a decade or more earlier, and generally seems to take place between the ages of 19 and 25 (Yanovski, 1993). BED is found to be more common in women than in men (3:2) and among patients attending weight loss treatment programs (Spitzer et al., 1992; Yanovski, 1993). In general, obese individuals with BED have an earlier onset of obesity than obese non-binge eaters ( 16.3 as compared to 25.1 years) and have a more unstable weight history (Brody, Walsh, \& Devlin, 1994). Furthermore, obese individuals with BED also report an earlier onset of dieting than obese nonbinge eaters (18.8 as compared to 26.4 years) (De Zwaan, Nutzinger, \& Schoenbeck, 1992). Episodes of weight cycling are significantly more frequent among those with BED than in the non BED group (Brody et al., 1994; Spitzer et al., 1992; Yanovski \& Sebring, 1994), there are more extensive histories of 'yo-yo' dieting (Wilson, Nonas, \& Rosenblum, 1993), and larger weight losses (15.1 kg versus $9.5 \mathrm{~kg}$ ) (Brody et al., 1994). Finally, the prevalence of serious problems with binge eating increases with the level of obesity (Bruce \& Agras, 1992; Telch, Agras, \& Rossiter, 1988; Telch \& Stice, 1998).

\section{CORE EATING DISORDER SYMPTOMATOLOGY OF OBESE BINGE EATERS AND OBESE NON-BINGE EATERS}

The core cognitive and behavioral eating disorder symptomatology of obese binge eaters and obese non-binge eaters are displayed in Table 1.2. Neither obese binge eaters nor obese non-binge eaters report high levels of dietary restraint (for a review, see Howard \& Porzelius, 1999). Obese binge eaters report objective binge eating episodes on a regular basis in the absence of compensatory behaviors, while obese non-binge eaters report these episodes less or not at all (Greeno, Wing, \& Marcus, 1999). In a study of Marcus and colleagues (1992) obese binge eaters mentioned binge episodes with an average duration of over 5 hours, and $23.5 \%$ reported binge episodes lasting all day (grazing). When instructed to binge eat, persons with BED consumed a greater 
Table 1.2 Core eating disorder symptomatology of obese binge eaters and obese non-binge eaters

\begin{tabular}{lll}
\hline Cognitive and behavioral features & Obese non-binge eaters & Obese binge eaters \\
\hline Restraint & Slight & Slight \\
Objective binge episodes & Slight & Strong \\
Chaotic eating pattern & Moderate & Strong \\
Body Image dissatisfaction & Moderate & Strong \\
Shape, weight, eating concerns & Moderate & Strong \\
Low self-esteem & Moderate & Strong \\
\hline
\end{tabular}

percentage of fat and a lower percentage of protein, than weight matched controls (Yanovski, 1992). Obese persons with and those without BED struggle to regulate their eating and report a chaotic eating pattern (Castonguay, Eldredge, \& Agras, 1995; Marcus et al., 1992; Rossiter et al., 1992). Yanovski and colleagues (1992) found that obese binge eaters consumed significantly more energy when they were asked to eat normally than obese non-binge eaters.

Body image is a multidimensional construct. It incorporates how an individual sees, experiences, thinks about, behaves and feels about his or her body shape and weight (Rosen, 1997). Problems of body image can range from mild body dissatisfaction to an extreme preoccupation with physical appearance that impairs functioning (Rosen, Orosan, \& Reiter, 1995). The vast majority of obese women demonstrated severe body image dissatisfaction related to their obesity (Adami et al., 1998; Cash, Counts, \& Huffine, 1990), with almost half reporting specific concerns over their waist or abdomen rather than global appearance-related concerns (Sarwer, Wadden, \& Foster, 1998). Obese women experienced significantly greater body image dissatisfaction than non-obese controls (Sarwer et al., 1998). Furthermore, body image dissatisfaction is higher in persons with BED than in obese non-binge eaters (Grilo, Wilfley, Jones, Brownell, \& Rodin, 1994; Lloyd-Richardson, King, Forsyth, \& Clark, 2000; Mussell et al., 1996; Striegel-Moore, Wilson, Wilfley, Elder, \& Brownell, 1998). In addition, obese binge eaters show more concerns about shape, weight, and eating than obese non-binge eaters (Eldredge \& Agras, 1996; Telch \& Stice, 1998; Wilfley, Schwartz, Spurrell, Fairburn, 2000; Wilson et al., 1993). BED patients are more similar to bulimia nervosa patients in their distorted attitudes towards shape, weight, and eating (Marcus et al., 1992; Masheb \& Grilo, 2000; Wilfley et al., 2000). Finally, obese binge eaters have lower self-esteem than obese non-binge eaters (De Zwaan et al., 1994; Striegel-Moore et al., 1998; Telch \& Agras, 1994; Telch \& Stice, 1998). 


\section{COMORBIDITY IN OBESE BINGE EATERS AND OBESE NON- BINGE EATERS}

Persons with BED show significantly more psychiatric comorbidity than obese non-binge eaters. Most studies have found that obese persons with BED who seek treatment are more vulnerable to depression than obese non-binge eaters (e.g., Hudson et al., 1988; Kuehnel \& Wadden, 1994; Marcus, Wing, \& Hopkins, 1988; Mussell et al., 1996; Specker, De Zwaan, Raymond, \& Mitchell, 1994; Striegel-Moore et al., 1998; Telch \& Agras, 1994; Wilfley et al., 2000; Yanovski, Nelson, Dubbert, \& Spitzer, 1993). Individuals with BED have significantly higher rates of lifetime Axis I comorbidity (ranging from $59 \%$ to $72 \%$ in various treatment-seeking and non-treatment seeking BED samples) as compared to non-binge eaters (Wilfley et al., 2000). Yanovski and colleagues (1993) found a lifetime diagnosis of major depression of $51 \%$ in obese binge eaters and of $14 \%$ in obese non-binge eaters. In a study of Mussell et al. (1996) $37 \%$ of persons with BED reported a lifetime diagnosis of major depression compared to $9 \%$ of obese non-binge eaters. Both obesity and binge eating may contribute to depression (Wadden \& Stunkard, 1985). Obesity is associated with stigmatization in Western society and this might be lead to depression. Furthermore, the sense of being out of control over eating that is seen in BED is highly aversive and may cause or exacerbate depressive symptomatology (Marcus et al., 1990). For most obese individuals, depression decreases after improvement of binge eating or obesity. Therefore, depression can be viewed as being secondary to the eating disorder (Smith, Marcus, \& Eldredge, 1994). Schwalberg and colleagues (1992) reported that $70 \%$ of obese binge eaters who seek treatment met lifetime criteria for a DSM-III-r anxiety disorder. In other studies a lifetime history of anxiety has been found in approximately $20 \%$ of obese binge eaters (Marcus et al., 1990; Mussell et al., 1996; Wilfley et al., 2000; Yanovski et al., 1993).Yanovski et al. (1993) found a greater lifetime

16 prevalence of panic disorder for obese binge eaters than for non-binge eaters ( $9 \%$ as compared to $1 \%$ ).

It would be reasonable to assume that obese persons with a comorbid Axis-II disorder are particularly challenging in therapy. In an interview-based study, Yanovski and colleagues (1993) found that individuals with BED reported more borderline personality than obese non-binge eaters (14\% versus $1 \%)$, and more avoidant personality disorder ( $7 \%$ versus $1 \%$ ). Of all obese binge eaters, $36 \%$ has a comorbid axis-II disorder. In another study of Specker et al. (1994), it was found that both cluster B and cluster C personality disorders have been shown to be more prevalent among BED than non-BED participants seeking weight loss treatment. In a recent study, Wilfley and colleagues (2000) found that $37 \%$ of patients with BED met the criteria for at least one personality disorder. Six percent were diagnosed with cluster A personality disorder, $12 \%$ with cluster B personality disorder, and $26 \%$ with cluster C personality disorder. Axis-II psychopathology was related to more severe binge eating and eating disorder psychopathology at baseline. Although overall presence of axis-II psy- 
chopathology did not predict outcome, presence of cluster B personality disorder predicted higher levels of binge eating at 1-year after treatment (Wilfley et al., 2000). Finally, in an article of Sansone, Wiederman, \& Sansone (2000), reviewing studies on the prevalence of borderline personality disorder (BPS) among obese individuals, it was found that the lowest rates of BPS were found in obese persons seeking weight loss in non-psychological programs or primary care settings. The highest rates for BPS were reported among obese persons in eating disorder and mental health settings. Prevalence rates for BPS in persons with BED varied from $6 \%$ to $30 \%$. Thus, a significant minority of obese individuals with BED suffer from BPS. Studies examining the prevalence of substance abuse among obese binge eaters report lifetime rates of $12 \%$ to $33 \%$ (Spitzer et al., 1993; Wilfley et al., 2000; Yanovski et al., 1993).

In sum, the comorbidity rates differ from study to study. This might be due to the differences in the definitions of binge eating among the studies, differences in diagnostic instruments, and the variability among the samples investigated. Despite these differences in definitions, diagnostic instruments and variability among the samples, there is strong evidence that binge eaters represent a distinct subgroup among the obese. Individuals with BED exhibit levels of psychopathology higher than those seen in obese individuals without binge eating problems (De Zwaan et al., 1994; Marcus et al., 1990; Mussell et al., 1996; Specker et al., 1994; Striegel-Moore et al., 1998; Yanovski et al., 1993).

\section{TRADITIONAL BEHAVIORAL THERAPY OF OBESITY}

Traditional behavioral therapy (TBT) of obesity involves the prescription of an energy reduced diet to achieve weight loss in a relatively short period. The first behavioral therapy was published almost forty years ago (Ferster, Nurnberger, \& Levitt, 1962). It was Stuart (1967) who reported a behavioral program for obesity in which eight obese women were treated with an individually tailored behavioral program resulting in an average weight loss of $17 \mathrm{~kg}$ (range 12-21 $\mathrm{kg}$ ) over a 12-month period. In this program the focus was on changing eating behaviors, and daily weighing. This successful study led to the systematic application of behavioral elements to the treatment of obesity. Studies in the early 1970 s based upon this approach typically involved 10-weeks of group treatment. Weight loss averaged around $0.5 \mathrm{~kg}$ per week. Furthermore, the relevance of exercise in weight loss treatment was noticed in the 1970s (Mahoney \& Mahoney, 1976).

Behavioral therapy gradually evolved. Essentially, current behavioral therapy programs for obesity consist of four key elements: self-monitoring, developing regular eating habits, stimulus control techniques, and reinforcement of altered behavior (Wilson \& Brownell, 1980). Self-monitoring of food and liquid 
Table 1.3 Summary analysis of selected studies from 1974 to 1990 providing treatment by traditional behovior therapy

\begin{tabular}{lccccc}
\hline & 1974 & 1978 & 1984 & $1985-1987$ & $1988-1990$ \\
\hline Number of studies included & 15 & 17 & 15 & 13 & \multicolumn{1}{c}{5} \\
Initial weight (kg) & 73.4 & 87.3 & 88.7 & 87.2 & 91.9 \\
Length of treatment (weeks) & 8.4 & 10.5 & 13.2 & 15.6 & 21.3 \\
Weight loss (kg) & 3.8 & 4.2 & 6.9 & 8.4 & 8.5 \\
Loss per week (kg) & 0.5 & 0.4 & 0.5 & 0.5 & 0.4 \\
Attrition (\%) & 11.4 & 12.9 & 10.6 & 13.8 & 21.8 \\
Length of follow-up (weeks) & 15.5 & 30.3 & 58.4 & 48.3 & 53.0 \\
Weight loss at follow-up (kg) & 4.0 & 4.1 & 4.4 & 5.3 & 5.6 \\
\hline
\end{tabular}

Source: Wadden \& Bartlett (1992).

intake, including overeating episodes, begins early in treatment and continues throughout, setting the basis for treatment sessions. Additional details that may be recorded include time and place of eating, and situational factors associated with overeating. Psychoeducation about nutrition is also provided. A third element involves stimulus control procedures. Avoiding strategies and delay strategies are introduced, often in combination with activities that are incompatible with overeating. A fourth element is reinforcement. Reinforcement of altered behavior supposedly leads to continuation of that behavior.

Table 1.3 depicts the evolution of traditional behavioral therapy from 1974 to 1990. Several developments are noteworthy. First, more recent behavioral approaches have included heavier patients (from 73 to $92 \mathrm{~kg}$ at entry). Second, 18 treatment duration has increased from 8 to 21 weeks. Third, attrition rates increased from $11 \%$ to $22 \%$. Fourth, probably due to the longer interventions the average weight loss after treatment has almost doubled from $3.8 \mathrm{~kg}$ to 8.5 $\mathrm{kg}$. Fifth, the follow-up period after treatment has increased from 15-weeks to 1 -year, and finally, the maintenance weight loss at follow-up changed from 4.0 $\mathrm{kg}$ to $5.6 \mathrm{~kg}$.

In the 1990s, virtually all behavioral therapy programs were delivered in groups of 10-20 participants and involved weekly sessions for 15-24 weeks. The behavioral program changed as well. Patients in programs were given strict calorie goals (usually between $1200-1500 \mathrm{kcal}$ ), exercise was prescribed and financial incentives were often utilized. 


\section{CRITICISM AT TRADITIONAL BEHAVIORAL THERAPY}

Even at an early stage, the appropriateness of traditional behavioral therapy focusing on weight loss were subject to caveats. Stunkard had a highly pessimistic view of obese persons and therapy: 'Most obese persons will not stay in treatment for obesity. Of those who stay in treatment most will not lose weight and of those who do lose weight, most will regain it' (Stunkard, 1958, p.79). Below, four main arguments against the appropriateness of traditional behavioral therapy focusing on large weight loss will be summarized.

\section{Long-term ineffectiveness}

Traditional behavioral therapy is generally successful in the short-term, but is often not very effective in maintaining weight loss in the long-term (e.g., Bennett, 1986; Garner \& Wooley, 1991; Wadden, 1993; Wilson, 1994; Wooley \& Garner, 1991). A great number of studies have shown that almost all participants returned to their baseline weights or eventually weighed even more than they did at the start of treatment (e.g., Garner \& Wooley, 1991; Jeffery et al., 2000; Perri et al., 1987; Perri, Nezu, Patti, \& McCann, 1989; Wilson, 1994; Wooley \& Garner, 1991). The time at which the weight regain occurred varied across studies, but the trend towards a return to baseline values is clear, even when specific maintenance strategies were implemented during the follow-up period. Four different maintenance strategies were given in a study of Perri and colleagues (1988). In spite of this, almost all conditions began to regain weight at 18-month follow-up - the longer the follow-up, the more striking this effect. In one study, a five-year follow-up showed that only $13 \%$ of subjects had succeeded in maintaining a loss of $5 \mathrm{~kg}$ or more (Wadden, Sternberg, Letizia, Stunkard, \& Foster, 1989). In other words, research has consistently indicated that traditional behavioral therapy do not have long-term positive outcomes on weight.

\section{Influence of genetic and biological factors}

In the 1980 s, several studies underlined the importance of genetic influences on obesity (Bouchard, Perusse, Leblanc, Tremblay, \& Theriault, 1988; Stunkard et al., 1986: Stunkard, Harris, Pedersen, \& McClearn, 1990). Estimates of the variance in Body Mass Index that can be explained by genetics range from $25 \%$ to $80 \%$, depending on the kinds of categories of relatives studied (Bouchard \& Perusse, 1993). For example, Stunkard et al. (1986) reported the adult weights of 540 Danish adoptees and found that their weights were more closely related to their biological parents, with whom they had no contact, than to their adoptive parents they had been living with. In another study of Stunkard and colleagues (1990), it was found that in monozygotic twins raised separately genetics accounted for 
$66 \%$ of the variance in body weight. In addition, biological mechanisms are seen as a cause in the maintenance of obesity. Although the notion of a 'set point' around which body weight was biologically regulated remained controversial (Spitzer \& Rodin, 1981), it became widely accepted that once obesity developed it was defended by the body (Stallone \& Stunkard, 1991).

Although genetic and biological factors may influence limits in losing weight ${ }^{1}$, it should be noted that genetic influence partly operates through behavioral mechanisms. Environmental factors, such as the parents, may influence the food intake and activity levels of their children.

\section{Psychological heterogeneity}

Another argument against behavioral therapy programs for obesity is that they are tailored to a biased sample of obese people. Obese individuals who seek weight loss treatment display a higher prevalence of psychopathology than obese persons not seeking treatment (Foster \& Wadden, 1994; Friedman \& Brownell, 1995; Miller \& Downey, 1999), and are the ones having most difficulty with losing weight (Brownell, 1992). Psychological distress itself might influence the decision to seek treatment. Moreover, obese binge eaters perform less well in behavioral programs than obese non-binge eaters. Obese binge eaters lose significantly less weight or rapidly regain it (Keefe, Wyshogrod, Weinberger, \& Agras, 1984; Marcus et al., 1988; Yanovski, Gormally, Leser, Gwirtsman, \& Yanovski, 1994), and more frequently drop out of treatment (Keefe et al., 1984; Marcus et al., 1988; Yanovski et al., 1994).

The assumption that all obese persons are similar resulted in treatment efforts employing a single approach. Furthermore, traditional behavioral therapy typically do not take into account the high level of psychopathology in obese persons seeking specialized treatment.

\section{Harmfulness}

Continued participation in traditional behavioral therapy is associated with strict dieting and repeated loss and regain of weight (weight cycling). It has been

\footnotetext{
I This is an argument against all psychological treatments. However, it seems that this argument applies more to traditional behavioral therapy focusing on weight loss based on weight tables in favor of what is described as 'reasonable weight'. In contrast, non-dieting cognitive-behavioral therapy for obese persons focus on selfacceptance, regardless whether they succeed in efforts at weight control. Enhancing self-acceptance may not only provide a more appropriate goal to what has proved a chronic problem, but might also lead to more lasting reductions in weight by helping obese persons to accept only modest weight loss and improve compliance with healthy eating and exercise behaviors (Devlin, Yanovski, \& Wilson, 2000; Wilson, 1996).
} 
suggested that this might lead to increased mortality and morbidity and adverse metabolic effects, psychological effects, and binge eating.

\section{Increased mortality and morbidity and adverse metabolic effects}

The weight cycling hypothesis was based on a landmark study of rats subjected to repeated cycles of weight loss and weight regain (Brownell, Greenwood, Stallar, \& Shrager, 1986).

Each successive cycle made weight loss more difficult and weight gain easier, suggesting some metabolic effects of 'yo-yo' dieting. The weight cycling hypothesis posits two effects of weight cycling. The first is that weight cycling increased mortality and morbidity. The second is that weight cycling decreases the resting metabolic rate and increases the proportion of body fat.

The first effect that has been examined is that weight cycling leads to increased mortality and morbidity. A number of epidemiological studies suggest that weight cycling is associated with a higher rate of morbidity and mortality (Blair, Shaten, Brownell, Collins, \& Lissner, 1993; Hamm, Shekelle, \& Stamler, 1989; Higgins, D'Agostino, Kannel, \& Cobb, 1993; Lee \& Paffenbarger, 1992; Lissner et al., 1991; Pamuk, Williamson, Serdula, Madans, \& Byers, 1993). However, one of the problems in interpreting this epidemiological data is to determine whether or not these cycles of weight loss were voluntary. It is possible that weight loss reflected an existing illness.

The second effect that has been examined is that weight cycling decreases the resting metabolic rate and increases the proportion of body fat. Some studies support the hypothesis that weight cycling decreases the resting metabolic rate and increases the proportion of body fat (Blackburn et al., 1989; Manore, Berry, Skinner, \& Caroll, 1991; Rodin, Radke-Sharpe, Rebuffé-Scrive, \& Greenwood, 1990; Steen, Oppliger, \& Brownell, 1988). However, several recent reviews on animal and human research conclude that the majority of available data provides little evidence to support adverse effects of weight cycling on metabolic rate, and fat distribution (National Task Force on the Prevention and Treatment of Obesity, 1994; Reed \& Hill, 1993; Wing, 1992).

\section{Psychological effects}

Negative effects of strict dieting and weight cycling may be psychological in nature. Since the classic studies by Keys and his colleagues (1950) on starvation, strict dieting has been postulated to lead to negative changes in cognitions and affect. Thirty-six young men who were healthy, were placed on a very low calorie diet for 6-months. It was shown that during the 6-months in which they lost approximately $25 \%$ of their weight, they experienced attitudinal, behavioral and emotional changes. Most men experienced periods of depression, anxiety, irritability and outbursts of anger. In addition, they found it increasingly difficult to concentrate on their usual activities because they became obsessed with eating and food concerns. A problem of this study is that the results may not be generalized to a female chronic dieter. So far, only few studies have focused on effects of strict dieting and weight cycling on psycho- 
logical factors (Brownell \& Rodin, 1994; Foster, Sarwer, \& Wadden, 1997). In a retrospective study of Wadden, Stunkard, \& Liebschutz (1988), it was found that weight regain had negative effects on participants' self-esteem, body image and relationships. Furthermore, dieting or weight cycling might result in pathological changes in cognition and affect (Brownell \& Rodin, 1994; Foreyt et al., 1995; Friedman \& Brownell, 1995; Polivy \& Herman, 1985; Stunkard, 1957). However, in a review on the psychological effects of weight cycling, it was found that weight cycling was not associated with psychopathology or depressogenic cognitions (Foster et al., 1997). Prospective and serial measurements during weight loss and regain will be necessary to provide more accurate information about the psychological effects of dieting and weight cycling.

\section{Binge eating}

Finally, it has been postulated that dieting lead to binge eating. Several retrospective studies have attempted to assess whether or not dieting precedes the onset of binge eating in obese persons. In such studies, the majority of individuals report that they had begun binge eating before dieting (Abbott et al., 1998; Malkoff, Marcus, Grant, Moulton, \& Vayonis, 1993; Mussell et al., 1995, Spurrell, Wilfley, Tanofsky, \& Brownell, 1997; Wilson et al., 1993). Two comparable studies showed that between $25 \%$ and $37 \%$ of participants reported dieting before binge eating, whereas between $49 \%$ and $54 \%$ of respondents reported binge eating before they began dieting (Mussell et al., 1995; Spurrell et al., 1997). Between $15 \%$ and $21 \%$ were unsure as to whether dieting or binge eating came first. Those who binge first had an earlier onset of obesity. Furthermore, the binge-first group reported more family problems, life events and psychiatric comorbidity (Abbott et al., 1998). A limitation of these retrospective studies is that person's memory of the distant past tends to be rather blurred and may be biased by the person's current perspective. In a prospective study of Telch and Agras (1993), it was found that $63 \%$ of obese women iden-

22 tified at baseline as non-binge eaters reported binge eating episodes after completion of a very low-calorie program. About $15 \%$ of those who began treatment without having binge problems first, met the full criteria for BED at final assessment. In sum, the studies are inconclusive as to the exact role played by dieting in the onset of binge eating. Dieting appears to play a significant role in the etiology of binge eating for some obese individuals but not for others (Howard \& Porzelius, 1999).

\section{Conclusions}

All in all, there are several arguments against traditional behavioral therapy. The first, and most important, is that research has consistently shown that traditional behavioral therapy was not successful in long-term weight loss. Furthermore, traditional behavioral therapy might have certain risks. Some studies suggest that weight cycling leads to increased mortality and morbidity. In addition, 
weight cycling decreases the resting metabolic rate and increases the proportion of body fat. Furthermore, strict dieting might result in pathological changes in cognitions and affect and might lead to binge eating. In addition, obese binge eaters, even to a greater extent than obese non-binge eaters, systematically report other problems than weight disturbances, for example, dysfunctional cognitions about shape, weight, and eating, and low self-esteem. The shortcomings of traditional behavioral therapy has led to the development of a nondieting cognitive-behavioral model and is discussed in the next Chapter. 


\section{A COGNITIVE-BEHAVIORAL MODEL FOR THE TREATMENT OF OBESITY AND BINGE EATING DISORDER}

\section{A COGNITIVE-BEHAVIORAL MODEL}

Researchers and clinicians are beginning to call for approaches that focus on outcome goals other than weight loss (Ciliska, 1990, 1998; Devlin et al., 2000; Garner \& Wooley, 1991; Polivy \& Herman, 1983, 1992; Wardle, 1995; Wilson, 1996). One of these approaches is the cognitive-behavioral therapy, which aims at improving psychological well-being, restructuring dysfunctional cognitions regarding body weight and shape, and on restoring normal and healthy eating patterns, rather than at weight loss per se. Obese persons are encouraged to accept themselves, regardless of their weight and of whether they succeed in efforts at controlling their weight. This approach is based on the idea that weight loss may occur when dieting is discontinued. Achieving a small weight loss for overweight individuals is enough, since this is a realistic level that can be maintained without strict dieting or excessive exercise. This approach is based on the assumption that obesity often has chronic courses (Wilson, 1999). The cognitive-behavioral approach consists of a behavioral (eating behavior) and a cognitive part. 


\section{Eating behavior}

The Externality Hypothesis by Schachter (Schachter, 1971) was one of the first theories that helped to rationalize behavioral therapy for obese individuals. Schachter (1971) found that eating behavior of the obese is stimulated by foodrelevant external cues, while the eating behavior of normal weight individuals is closely tied to internal state. However, subsequent investigations were unable to confirm that obese individuals are more responsive to external cues and less responsive to internal cues than normal weight individuals (Rodin, 1981; Ruderman, 1986). Therefore, the internal versus external concept seems far too simple to explain differences in eating behavior.

Nisbett (1972) argued that external responsiveness was not related to obesity per se, but was a consequence of caloric deprivation. He proposed that weight is regulated about a genetic 'set point'. This set point was assumed to be higher for obese individuals than for normal weight controls. Obese individuals who tried to lose weight hold their weight below a biologically dictated set point and are in a constant moderate state of hunger. This deprivation was expected to result in external responsiveness.

In later studies, external responsiveness was assumed to be a correlate of restraint. The construct of restraint was developed by Herman and Mack (1975). Restraint was defined as a cognitive tendency to restrict food intake in order to control weight (Herman \& Polivy, 1980). Herman and Mack (1975) hypothesized that normal weight dieters should exhibit eating patterns similar to obese individuals. To test this hypothesis they asked normal weight college women classified as restrained or unrestrained eaters to rate different flavors of ice cream (taste test) after being exposed to one of two different conditions. In one condition participants were given a 'diet-breaking' milkshake preload, while in the other condition the taste test was not preceded by a milkshake preload. It was found that restrained eaters ate more after having consumed a milkshake preload ('counterregulation'), whereas unrestrained eaters ate less after the milkshake preload. To demonstrate that dieting status regardless of weight produced this counterregulatory eating pattern, Hibscher and Herman (1977) conducted a study using obese, normal, and underweight college males. They were divided into restrained and unrestrained eaters. Consumption following a preload was the result of restraint, not obesity per se, with restrained eaters consuming more after eating a high caloric preload.

In an effort to further explain the relationship between restraint and external responsiveness, Herman and Polivy (1983) hypothesized that restrained eaters create a 'diet boundary' to regulate their eating in order to achieve their weight loss goals. The boundary consists of rigid cognitive rules concerning permissible levels of food intake. The permissible levels of food intake are lower than is needed for physiological satiety. In other words, cognitive control overrides the physiological control over food intake. If the diet boundary is transgressed, for example by consuming high caloric food, the individual begins to feel a sense of failure, loses control and begins to eat until feeling uncomfortably full. 
If it is true that restraint increases binge eating, persons with BED should have higher scores on the measure of dietary restraint than persons without BED. Several studies have investigated the relationship between binge eating severity and the level of dietary restraint. Most studies found low to moderate levels of restraint in obese binge eaters and obese non-binge eaters (Yanovski \& Sebring, 1994; Wilfley et al., 2000; Wilson et al., 1993). Additionally, in a survey of obese binge eaters and non-binge eaters, Wilson and colleagues (1993) found that obese binge eaters expressed greater preoccupation with poor eating control, yet no differences were found for food restriction. Furthermore, Howard and Porzelius (1999) also suggested that obese binge eaters as compared to obese non-binge eaters tend to be more preoccupied with poor eating control; and in addition, they have a greater desire to lose weight, and set unrealistically high standards for sustaining a diet. All of these dieting concerns might lead to binge eating in obese binge eaters and to overeating in obese non-binge eaters.

Further empirical studies in which the onset of binge eating episodes is examined also raise questions about the role of dietary restraint. Several retrospective studies found that about $50 \%$ of the obese binge eaters report that they began binge eating before dieting (Abbott et al., 1998; Malkoff et al., 1993; Mussell et al., 1995; Spurrell et al., 1997; Wilson et al., 1993). However, in a prospective study of Telch and Agras (1993), it was found that $63 \%$ of obese women identified at baseline as non-binge eaters reported binge eating episodes after completion of a very low-calorie program.

On the whole, studies are inconclusive as to the exact role of restraint in the onset of binge eating in obese binge eaters and obese non-binge eaters. Restraint does play a role in the etiology of binge eating for some obese individuals but not for others. The assumption that restraint leads to overeating and binge eating is not sufficient. It might be that dieting concerns such as preoccupation with poor eating control, a desire to lose weight, and unrealistically high standards for sustaining a diet lead to binge eating in obese binge eaters and to overeating in obese non-binge eaters. Some authors have suggested other extensions for the restraint model for obesity. Craighead and Allen (1995) and Allen and Craighead (1999) have emphasized the role of negative affect that can precipitate binge eating, even in the absence of a restrictive eating pattern. A study among obese females seeking treatment for binge eating showed that a negative mood without conditions of deprivation leads to binge eating (Arnow, Kenardy, \& Agras, 1992). Furthermore, two laboratory experiments were conducted with obese binge eaters (Agras \& Telch, 1998) and obese persons with and without binge eating (Telch \& Agras, 1996) in which they were assigned to a negative or neutral mood induction procedure prior to being served different food products. It was found that a negative mood increased the occurrence of binge eating. Finally, Greeno and colleagues (2000) studied antecedents of binge eating episodes. Women with BED and weight- and age-matched comparable women without BED monitored their food intake for 6 days, using handheld computers to measure mood, appetite, and setting at all eating episodes. 
Both negative mood, and preoccupation with poor eating control preceded binge episodes for the BED group. Current modifications in the restraint model for obesity might acknowledge the role of negative affect and dieting concerns.

\section{Cognitions}

Cognitive theory of obesity describes the influence of cognitions on the development and maintenance of disturbed eating and weight control behaviors. In cognitive theory schemas are seen as central explanatory concepts. Schemas are defined as a 'stored body of knowledge' which purpose is to direct attention, perception and information processing (Vitousek \& Hollon, 1990). Schemas are presumed for all information that requires processing, organizing, and integrating, and can serve functional as well as dysfunctional purposes (Williamson, Muller, Reas, \& Thaw, 1999).

In individuals with anorexia nervosa (Garner \& Bemis, 1982) and bulimia nervosa (Fairburn, 1985; Fairburn, Marcus, \& Wilson, 1993), it is assumed that they have developed a dysfunctional schema that centers on overconcern with weight and shape. Self-evaluation is influenced by how someone perceives his or her weight and shape. This might also be true in some people with obesity, especially obese persons with intense shape and weight concerns and in individuals with BED. When the body schema is activated, ambiguous stimuli are biased. The cognitive bias to body weight and shape leads to distortions in attention, judgment, memory and body image and could ultimately lead to disturbed eating patterns and weight control behaviors (Williamson et al., 1999).

The cognitive theory includes a hierarchy of cognitions labeled the "controlling cognitive constellation' (Beck, 1976). The most fundamental level consists of self-schemas or core beliefs. Self-schemas consist of beliefs about the self (e.g., worthless, helpless, loveless) and a primitive view of others (e.g., hostile, rejecting, demanding). The conditional assumptions corresponding to 'if ... then' behavioral rules (Beck, 1976), stipulates the criteria under which the selfschemas are applicable, and, thus, become activated. Self-schemas are balanced by compensatory assumptions such as 'If I am thin, I can avoid rejection'. These compensatory assumptions lead to imperatives such as 'I always have to diet'. Finally, automatic thoughts are spontaneous and operates outside immediate awareness, but can be identified.

Empirical evidence for the cognitive theory of obesity and BED is in its infancy. Two methodological approaches have been followed to test the cognitive theory. First, examination of the thought content, and second, examination of the cognitive processes.

In the examination of thought content in obesity, two general research methodologies have been used: in vivo data collection and retrospective selfstatement questionnaires. Hunt and Rosen (1981) used a time-sampling procedure and collected self-monitored cognitions from normal-weight and obese females. No group differences were found with respect to the frequency and 
quality of food, eating, and body-image related thoughts. O'Connor and Dowrick (1987) asked normal-weight, obese, and previously obese people to rate belief in and frequency of 26 dysfunctional cognitions concerning weight, food and eating. For example 'I have absolutely no self-control when it comes to food'. Obese participants reported higher beliefs in dysfunctional food and weight-focused cognitions than normal weight people. In other studies, selfstatement questionnaires designed for eating disorders were administered to obese subjects. Phelan (1987), for example, used the Bulimic Thoughts Questionnaire and found that obese subjects and eating disordered subjects scored similarly on cognitions about their ability to maintain a desirable weight. However, eating disordered subjects scored higher on cognitions about the unrealistic expectations of what would happen if 'forbidden food' was consumed, as well as about being out of control with food. In sum, the studies mentioned suggest that obese persons possess more dysfunctional cognitions than normal-weight persons, but have less pathologic scores on several measures of cognitions related to weight, shape, food, and eating than eating disordered subjects.

In addition, the subgroup of obese binge eaters shows more concerns about shape, weight, and eating than obese non-binge eaters (Eldredge \& Agras, 1996; Telch \& Stice, 1998; Wilfley et al., 2000; Wilson et al., 1993). Individuals with BED are similar to bulimia nervosa patients in their distorted cognitions towards shape, weight, and eating (Marcus et al., 1992; Masheb \& Grilo, 2000; Wilfley et al., 2000).

Findings from research on cognitive processes, such as attentional bias, are presumed to reflect the operation of schemas. Studies investigating cognitive processes in obesity and BED are very scarce. In an experimental design in normal-weight, overweight, and obese individuals, Markus, Hamill, and Sentis (1987) investigated self-schemas belonging to body weight for their effects on methods of processing weight-relevant information. They found that the objective weight status of an individual did not correspond to the method of processing weight-relevant information, but did correspond to the intensity in with which an individual is concerned about body weight and about a disposition to characterize weight as an organizing feature of the self.

Attentional bias refers to increased sensitivity to and absorption of relevant environmental cues. Attentional bias in eating disorders has generally been assessed using the Stroop Color Naming test (Mathews \& MacLeod, 1985). Studies have shown that patients with anorexia nervosa and bulimia nervosa are slower to color-name words relevant to eating, weight and shape concerns than normal controls (for a review, see, for example Cooper, 1997). One study included obese restrained eaters (Long, Hinton, \& Gillespie, 1994), and found that patients with anorexia nervosa did not differ from obese restrained eaters in color-name words relevant to food and body size. No such studies have further been conducted with obese binge eaters or obese non-binge eaters.

Negative body image seems to be present in individuals suffering from BED and in some obese persons (Rosen, Orosan, \& Reiter, 1995). In recent years, a 
number of researchers have suggested that negative body image is best conceptualized as the result of a combination of cognitive biases (Vitousek \& Hollon, 1990; Williamson, Cubic, \& Gleaves, 1993). Stunkard \& Mendelsohn were the first to report that some obese persons suffered from body image dissatisfaction. Body image dissatisfaction takes the form of an extreme preoccupation with one's body shape and weight, often to the exclusion of any other personal characteristics. 'It makes no difference whether the person be also talented, wealthy, or intelligent: his weight is his only concern, and he sees the whole world in terms of body weight' (Stunkard \& Mendelsohn, 1961, p. 328). Recent studies have confirmed body image dissatisfaction in a large proportion of obese persons (Adami et al., 1998; Cash et al., 1990; Collins et al., 1987; Sarwer et al., 1998). Sarwer and colleagues (1998) found that more than half of the 79 obese participants in their study mentioned that they were dissatisfied with their appearance and attempted to camouflage their body from others. Additionally, almost half of the obese individuals reporting specific concerns about their waist or abdomen rather than global appearance related concerns (Sarwer et al., 1998). Furthermore, body image dissatisfaction is higher in persons with BED as compared to obese non-binge eaters (Grilo et al., 1994; Lloyd-Richardson et al., 2000; Mussell et al., 1996; Striegel-Moore et al., 1998).

\section{A proposed cognitive-behavioral model for obese binge eaters and obese non-binge eaters}

On the basis of theories and ideas about disturbed eating patterns and dysfunctional cognitions, a speculative model might be proposed for obese binge eaters and obese non-binge eaters. This model is strongly based upon a model for bulimia nervosa patients outlined by Fairburn and colleagues (1993). In their model extreme concerns about body weight and shape play a central role. These concerns lead bulimics to restrict their food intake in rigid and unrealistic ways, which makes them physiologically and psychologically sensitive to periods of loss of control over eating. Binge eating, in turn, leads to strict dieting, extreme concerns about shape and weight, and low self-esteem. These conditions, in turn, will lead to more dietary restraint and binge eating. Finally, because their model explains the maintenance of bulimia nervosa, binge eating leads to compensatory purging behavior. Purging behavior, vice versa, leads to binge eating and low self-esteem (Fairburn et al., 1993; Wilson, Fairburn, \& Agras, 1997).

A number of alterations have been made to make this model applicable to obese binge eaters and obese non-binge eaters. Obese persons have many features in common with patients with bulimia nervosa, especially their concerns about shape and weight (Marcus et al., 1992; Masheb \& Grilo, 2000; Wilfley et al., 2000). This part of the model remains important. Self-evaluation is influenced by how someone perceives her body size and shape. Negative attitudes towards obese persons are widespread in Western countries and obese persons are one 


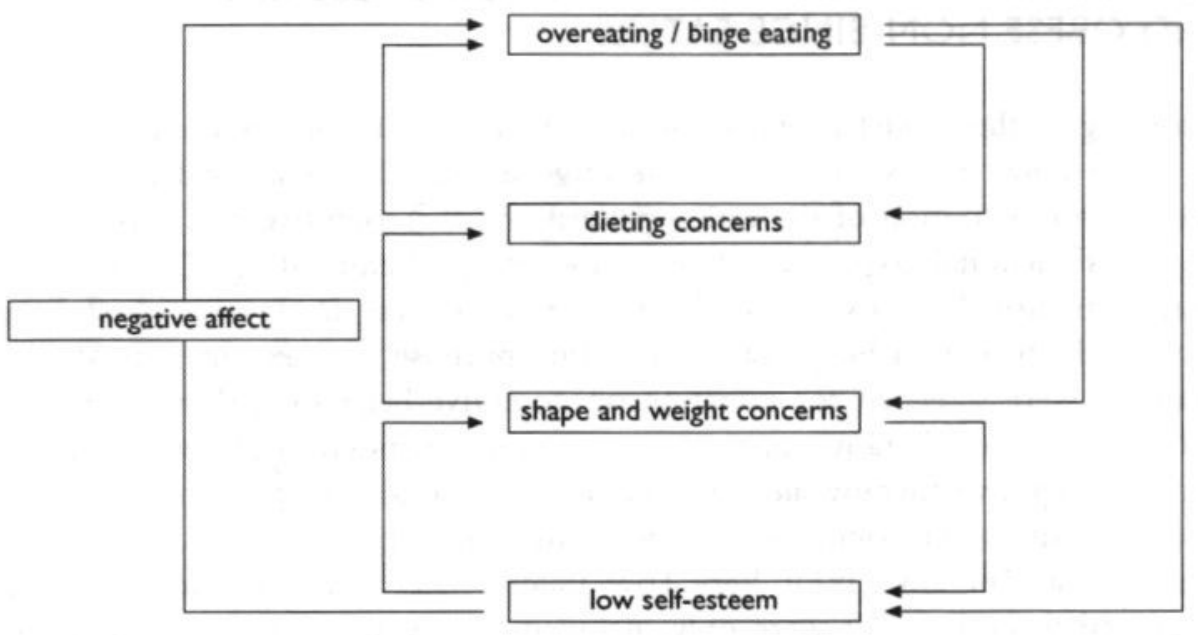

Figure 2.I Cognitive-behovioral model for obese binge eaters and obese non-binge eaters

of the stigmatized groups and this might give rise to low self-esteem and thereby negative mood (Castonguay et al., 1995). Most studies found that obese binge eaters are more vulnerable to depression than obese non-binge eaters (e.g., Mussell et al., 1996; Specker et al., 1994; Striegel-Moore et al., 1998; Telch \& Agras, 1994; Wilfley et al., 2000). A negative mood may directly trigger periods of loss of control over eating (Agras \& Telch, 1998; Arnow et al., 1992; Greeno et al., 2000; Telch \& Agras, 1996). Extreme concerns about body weight and shape lead bulimics to restrict their food intake in rigid and unrealistic ways. Studies are inconclusive as to the exact role played by restraint in the onset of binge eating or overeating in obese binge eaters and obese non-binge eaters (Howard \& Porzelius, 1999). Most obese persons do not report high levels of food restriction (Yanovski \& Sebring, 1994; Wilfley et al., 2000; Wilson et al., 1993). However, dieting concerns (preoccupation with poor eating control, intense desire to lose weight, and unrealistically high standards for sustaining a diet) might lead to overeating in obese non-binge eaters and, because of the more intense preoccupations in obese binge eaters, to overeating and binge eating in this subgroup. Overeating and, particularly, the sense of being out of control over eating seen in obese binge eaters is highly aversive. This may cause or exacerbate preoccupation with eating and weight loss, extreme concerns about shape and weight, and low self-esteem. Finally, obese persons do not engage in purging behavior, so this part of the model has been omitted. The model for obese binge eaters and obese non-binge eaters is illustrated in Figure 2.1. It is beyond the scope of this thesis to test this model empirically. However, this model underlies the cognitive and behavioral therapy mentioned in this thesis. 


\section{COGNITIVE-BEHAVIORAL THERAPY FOR OBESE BINGE EATERS AND OBESE NON-BINGE EATERS}

According to the model cognitive-behavioral therapy is the preferred treatment for obese binge eaters and obese non-binge eaters. The behavioral part should establish normalization of the eating pattern and the cognitive part should challenge dysfunctional cognitions about shape, weight, and eating. The following paragraphs provide an overview of cognitive-behavioral therapy for obese binge eaters and obese non-binge eaters. For this purpose, the Psyclit and Medline databases were screened for literature on cognitive-behavioral therapy for BED and obesity. The therapy approaches reviewed included cognitive-behavioral therapy, cognitive therapy and non-dieting behavioral therapy. Since the focus in this thesis is on cognitive-behavioral therapy, pharmacological treatment, InterPersonal Therapy (IPT), Very Low Calorie Diet (VLCD), and Traditional Behavioral therapy (TBT) were only included if they were compared to cognitive therapy (CT) or cognitive-behavioral therapy (CBT). CBT is a broad form of therapy. For this overview, all forms of therapy with a mainly cognitive focus such as non-dieting treatment, body image therapy, and appetite awareness training were included as CBT. The most common outcome measures were weight loss and/or bingeing. Some studies included other psychological outcome measures. The outcome measures most frequently used were depression, self-esteem, restraint, and eating pathology. Therefore, these measures were also included if mentioned in the studies. Cognitive-behavioral therapy will be reviewed for obese non-binge eaters, obese binge eaters, and both groups, respectively.

\section{Cognitive-behavioral therapy for obese non-binge eaters}

32 Six (un)controlled studies have investigated the effects of CBT or CT on obese non-binge eaters (Ciliska, 1990; Collins, Rothblum, \& Wilson, 1986; Polivy \& Herman, 1992; Rosen et al., 1995; Sbrocco, Nedegaard, Stone, \& Lewis, 1999; Tanco, Linden, \& Earle, 1998). See Table 2.1 for an overview.

Treatment duration of CBT or CT ranged from 8 to 13 weeks. Attrition rates ranged from $8 \%$ to $20 \%$. At posttreatment mean weight gain in CBT or CT was $0.3 \mathrm{~kg}$ and at follow-up mean weight loss was $2.3 \mathrm{~kg}$. At posttreatment mean weight gain in the waiting list or minimal control group was $0.4 \mathrm{~kg}$, and in TBT a mean weight loss of $2.8 \mathrm{~kg}$ was found. The follow-up data of TBT showed a mean weight loss of $3.5 \mathrm{~kg}^{2}$. 
Table 2.I Studies of cognitive-behavioral therapy for obese non-binge eaters (NB)

\begin{tabular}{|c|c|c|c|c|c|c|c|}
\hline $7 x+52$ & $\begin{array}{l}\text { Treatment } \\
\text { Components }\end{array}$ & $\begin{array}{l}\text { Sample } \\
\text { size }\end{array}$ & $\begin{array}{l}\text { Drop- } \\
\text { outs }\end{array}$ & $\begin{array}{l}\text { Treatment } \\
\text { response } \\
\text { weight loss }\end{array}$ & $\begin{array}{l}\text { Follow-Up } \\
\text { (FU) }\end{array}$ & $\begin{array}{l}\text { FU-response } \\
\text { weight loss }\end{array}$ & $\begin{array}{l}\text { Other psychological } \\
\text { measures }\end{array}$ \\
\hline $\begin{array}{l}\text { (A) Uncontrolled studies } \\
\text { Polivy \& Herman } \\
\text { (1992) }\end{array}$ & $\begin{array}{l}\text { CBTI (non-dieting treatment) } \\
\text { (10 sessions) }\end{array}$ & $18 \mathrm{NB}$ & $17 \%$ & $+5.7 \mathrm{~kg}$ & 6 months & $+3.6 \mathrm{~kg}$ & $\begin{array}{l}\text { depression, self-esteem, } \\
\text { eating pathology (EDI2), } \\
\text { and restraint } \\
\text { significant improvement }\end{array}$ \\
\hline \multirow[t]{3}{*}{$\begin{array}{l}\text { (B) Controlled } \\
\text { (comparative) studies } \\
\text { Ciliska (1990) }\end{array}$} & $\begin{array}{l}\text { CBT (non-dieting treatment) } \\
\text { (12 sessions) }\end{array}$ & 49 NB & $14 \%$ & $+1.4 \mathrm{~kg}$ & $\begin{array}{l}6 \text { months/ } \\
1 \text { year }\end{array}$ & $\begin{array}{l}+1.3 / \\
-0.6 \mathrm{~kg}\end{array}$ & $\begin{array}{l}\text { depression, self-esteem, } \\
\text { eating pathology (EDI), } \\
\text { restraint } \\
\text { CBT> education }\end{array}$ \\
\hline & $\begin{array}{l}\text { Education } \\
\text { (12 sessions) }\end{array}$ & 47 NB & $23 \%$ & $-1.0 \mathrm{~kg}$ & & $\begin{array}{l}+5.4 l \\
0.0 \mathrm{~kg}\end{array}$ & \\
\hline & Waiting list & $46 \mathrm{NB}$ & $41 \%$ & $+0.2 \mathrm{~kg}$ & & - & \\
\hline
\end{tabular}


Table 2.1 Continued

\begin{tabular}{|c|c|c|c|c|c|c|c|}
\hline Author & $\begin{array}{l}\text { Treatment } \\
\text { Components }\end{array}$ & $\begin{array}{l}\text { Sample } \\
\text { size }\end{array}$ & $\begin{array}{l}\text { Drop- } \\
\text { outs }\end{array}$ & $\begin{array}{l}\text { Treatment } \\
\text { response } \\
\text { weight loss }\end{array}$ & $\begin{array}{l}\text { Follow-Up } \\
\text { (FU) }\end{array}$ & $\begin{array}{l}\text { FU-response } \\
\text { weight loss }\end{array}$ & $\begin{array}{l}\text { Other psychological } \\
\text { measures }\end{array}$ \\
\hline Collins & $\mathrm{CT}^{3}$ & $15 \mathrm{NB}$ & & & 7 months $^{5}$ & & \\
\hline \multirow[t]{11}{*}{ et al. (1986) } & (8 sessions treatment + & & $13 \%$ & $-2.5 \mathrm{~kg}$ & & $-5.3 \mathrm{~kg}$ & no additional \\
\hline & 12 months maintenance) $)^{4}$ & & $53 \%$ & & & & outcome measures \\
\hline & TBT6 & $15 \mathrm{NB}$ & & & & & \\
\hline & ( 8 sessions treatment + & & $20 \%$ & $-4.7 \mathrm{~kg}$ & & $-6.6 \mathrm{~kg}$ & \\
\hline & I 2 months maintenance) & & $26 \%$ & & & & \\
\hline & CBT & $15 \mathrm{NB}$ & & & & & \\
\hline & ( 8 sessions treatment + & & $20 \%$ & $-4.6 \mathrm{~kg}$ & & $-7.6 \mathrm{~kg}$ & \\
\hline & I2 months maintenance) & & $26 \%$ & & & & \\
\hline & Minimal Treatment & $15 \mathrm{NB}$ & & & & & \\
\hline & ( 8 sessions treatment + & & $33 \%$ & $-2.0 \mathrm{~kg}$ & & $-3.3 \mathrm{~kg}$ & \\
\hline & 12 months maintenance) & & $26 \%$ & & & & \\
\hline \multirow{4}{*}{$\begin{array}{l}\text { Rosen } \\
\text { et al. } 1995\end{array}$} & CBT (body image therapy) & $25 \mathrm{NB}$ & $7 \%$ & $+2.1 \mathrm{~kg}$ & 4.5 months & $+1.6 \mathrm{~kg}$ & self-esteem, body image \\
\hline & (8 sessions) & & & & & & and restraint \\
\hline & & & & & & & $\mathrm{CBT}>\mathrm{WL}$ \\
\hline & Waiting list & $23 \mathrm{NB}$ & $4 \%$ & $+1.9 \mathrm{~kg}$ & & & \\
\hline
\end{tabular}




\section{Table 2.1 Continued}

\begin{tabular}{|c|c|c|c|c|c|c|c|}
\hline Author & $\begin{array}{l}\text { Treatment } \\
\text { Components }\end{array}$ & $\begin{array}{l}\text { Sample } \\
\text { size }\end{array}$ & $\begin{array}{l}\text { Drop- } \\
\text { outs }\end{array}$ & $\begin{array}{l}\text { Treatment } \\
\text { response } \\
\text { weight loss }\end{array}$ & $\begin{array}{l}\text { Follow-Up } \\
\text { (FU) }\end{array}$ & $\begin{array}{l}\text { FU-response } \\
\text { weight loss }\end{array}$ & $\begin{array}{l}\text { Other psychological } \\
\text { measures }\end{array}$ \\
\hline Sbrocco & CBT (behavioral choice treatment) & $12 \mathrm{NB}$ & $8 \%$ & $-2.5 \mathrm{~kg}$ & 6 months/ & $-7.0 \mathrm{~kg} /$ & self-esteem \\
\hline \multirow[t]{4}{*}{ et al. (1999) } & (13 sessions) & & & & I year & $-10.0 \mathrm{~kg}$ & $\mathrm{CBT}=\mathrm{TBT}$ \\
\hline & & & & & & & $\begin{array}{l}\text { depression, } \\
\text { eating pathology (EDI), }\end{array}$ \\
\hline & TBT & $12 \mathrm{NB}$ & $0 \%$ & $-6.7 \mathrm{~kg}$ & & $-4.5 \mathrm{~kg} /$ & and restraint \\
\hline & (13 sessions) & & & & & $-4.3 \mathrm{~kg}$ & $\mathrm{CBT}>\mathrm{TBT}$ \\
\hline \multirow{6}{*}{$\begin{array}{l}\text { Tanco } \\
\text { et al. (1998) }\end{array}$} & CT & 20 NB & $10 \%$ & $-2.2 \mathrm{~kg}$ & 6 months & $-5.9 \mathrm{~kg}$ & depression and eating \\
\hline & (8 sessions) & & & & & & pathology (EDI) \\
\hline & & & & & & & $\mathrm{CT}>\mathrm{TBT}$ \\
\hline & TBT & $21 \mathrm{NB}$ & $10 \%$ & $-3.4 \mathrm{~kg}$ & & $-8.9 \mathrm{~kg}$ & \\
\hline & (8 sessions) & & & & & & \\
\hline & Waiting list & $19 \mathrm{NB}$ & $32 \%$ & $+0.8 \mathrm{~kg}$ & & - & \\
\hline
\end{tabular}

$1 \mathrm{CBT}=$ Cognitive Behavioral Therapy; ${ }^{2}$ Eating Disorder Inventory; ${ }^{3} \mathrm{CT}=$ Cognitive Therapy: ${ }^{4}$ Group met at 2 -months interval; 5 Data of 12 months Follow-Up were not available due to a very low attendance; 6 TBT $=$ Traditional Behavioral Therapy 
Uncontrolled CBT. There has been one uncontrolled study of a ten-week group CBT (Polivy \& Herman, 1992). This study found that weight did not improve directly after treatment and throughout the 6-month follow-up period. However, the other outcome measures in this study showed more encouraging results. Participants significantly reduced eating pathology and depression, and enhanced self-esteem.

CBT or CT versus waiting list or minimal treatment. Four studies have compared CBT or CT to a waiting-list control group or a minimal treatment (Ciliska, 1990; Collins et al., 1986; Rosen et al., 1995; Tanco et al., 1998). Two studies found at posttreatment no significant differences on weight loss (Ciliska, 1990; Rosen et al., 1995). In the study of Collins et al. (1986), CBT and CT were compared to a minimal treatment control. It was found that CBT was slightly superior in weight loss and no significant results were found for individuals in the CT. Tanco and colleagues' (1998) study showed that CT was significantly more effective in losing weight than a waiting list control group. Three of the studies also mentioned other outcome measures and found that depression, self-esteem, body image or eating pathology had significantly improved after CBT and CT (Ciliska, 1990; Rosen et al., 1995; Tanco et al., 1998).

Cognitive-behavioral therapy versus traditional behavioral treatment. Four studies have investigated the effects of TBT in comparison to CBT or CT (Ciliska, 1990; Collins et al., 1986; Sbrocco et al., 1999; Tanco et al., 1998). In the study of Ciliska (1990), education and CBT had no significant effects on weight at posttreatment and 1-year follow-up. A study of Sbrocco and colleagues (1999) showed that TBT in comparison to CBT evidenced greater weight loss at posttreatment. In addition, only CBT participants continued with weight loss at follow-up. In a study of Collins and colleagues (1986) CBT and TBT participants were more effective in reducing weight than CT participants. At 7months follow-up the three groups showed a continuing weight loss and there were no significant differences between groups. However, only CT participants tended to a continuing decrease in weight at follow-up. Tanco and colleagues (1998) found that both CT and TBT participants lost weight at posttreatment and continued to lose weight at 6-month follow-up. Furthermore, all studies using broad-spectrum outcomes found that individuals who received CT or CBT clearly benefited in terms of reduced eating pathology and depression and enhanced self-esteem. In contrast, individuals participating in a TBT did not show significant changes on these psychological variables (Ciliska, 1990; Sbrocco et al., 1999; Tanco et al., 1998).

In sum, the results for weight loss are somewhat inconclusive. In general it seems that in the short-term TBT might be more promising than CBT in reducing weight. However, at long-term follow-up no differences were found between CBT and TBT. Furthermore, an interesting result found in the studies is that CBT might have broader effects than TBT and could also improve depressive feelings, eating pathology and self-esteem. 


\section{Cognitive-behavioral therapy for obese binge eaters}

Eight studies have investigated the effects of CBT or CT on obese binge eaters (Agras et al., 1994; Agras et al., 1995; Allan \& Craighead, 1999; Goodrick, Poston, Kimball, Reeves, \& Foreyt, 1998; Marcus, Wing, \& Fairburn, 1995; Smith, Marcus, \& Kaye, 1992; Telch, Agras, Rossiter, Wilfley, \& Kenardy, 1990; Wilfley et al., 1993). See Table 2.2 for an overview.

The treatment duration ranged from 8 to 24 sessions. Attrition rates ranged from $11 \%$ to $33 \%$, with a mean attrition rate of $20 \%$. At posttreatment, participants in CBT showed a mean weight gain of $1.1 \mathrm{~kg}$. TBT participants lost 1.0 $\mathrm{kg}$ and waiting list participants gained $1.0 \mathrm{~kg}$. At posttreatment, CBT participants had reduced the frequency of bingeing by $76 \%$ and had an abstinence rate from $55 \%$. TBT had reduced bingeing by $44 \%$, and the waiting list group had reduced binge eating by $7 \%$ and had an abstinence rate of $4 \%$. Most studies did not report follow-up results for weight loss and binge eating.

Uncontrolled CBT. Smith and colleagues (1992) evaluated a 16-week CBT. They reported a $50 \%$ abstinence rate and a $81 \%$ reduction in binge eating after treatment. The weight loss was $1.1 \mathrm{~kg}$. A strong feature of the study of Smith and colleagues (1992) is that they measured shape, weight, and eating concerns. After treatment, significant changes in associated eating pathology and depression were shown.

CBT versus waiting list. Six studies have compared $\mathrm{CBT}$ to a waiting list and found that CBT was superior (Agras et al., 1995; Allan \& Craighead, 1999; Goodrick et al., 1998; Marcus et al., 1995; Telch et al., 1990; Wilfley et al., 1993). All but one study reported reductions in binge eating. At posttreatment, subjects who had received $C B T$ had significantly reduced their binge eating to a greater extent than subjects in the waiting list condition. Two studies mentioned follow-up results of binge eating (Telch et al., 1990; Wilfley et al., 1993). The study of Telch et al. (1990) included a 10-week follow-up period. Forty-six percent of the participants who received CBT were abstinent from binge eating and there was a $69 \%$ reduction in binge eating. In the study of Wilfley and colleagues (1993) there was a 1-year follow-up period with a $55 \%$ reduction of binge eating.

The outcome measure weight was reported in five of the six studies. In all studies it was found that after CBT participants kept the same weight or gained weight. For the outcome measure weight, there was no significant difference between CBT and the waiting list. Both conditions had no effect on weight. Long-term follow-up results for CBT were mentioned by two studies (Goodrick et al., 1998; Wilfley et al., 1993). At 1-year follow-up, these studies reported a weight gain of $1.2 \mathrm{~kg}$ for participants in the CBT and no weight gain $(0.0 \mathrm{~kg})$, respectively. In sum, the studies suggest that $\mathrm{CBT}$ as compared to a waiting list is more effective in reducing the frequency of binge eating, but there is no effect for weight. CBT leads to abstinence in about $50 \%$ of BED patients and to a mean reduction of binge eating of $76 \%$. 
Table 2.2 Studies of cognitive-behavioral therapy for obese binge eaters (B)

\begin{tabular}{|c|c|c|c|c|c|c|c|c|c|}
\hline Author & $\begin{array}{l}\text { Treatment } \\
\text { Components }\end{array}$ & $\begin{array}{l}\text { Sample } \\
\text { size }\end{array}$ & $\begin{array}{l}\text { Drop- } \\
\text { outs }\end{array}$ & $\begin{array}{l}\text { Treatment } \\
\text { response } \\
\text { weight loss }\end{array}$ & $\begin{array}{l}\text { Treatment } \\
\text { response } \\
\text { bingeing }\end{array}$ & $\begin{array}{l}\text { Follow-Up } \\
\text { (FU) }\end{array}$ & $\begin{array}{l}\text { FU-response } \\
\text { weight loss }\end{array}$ & $\begin{array}{l}\text { FU-response } \\
\text { bingeing }\end{array}$ & $\begin{array}{l}\text { Other } \\
\text { psychological } \\
\text { measures }\end{array}$ \\
\hline $\begin{array}{l}\text { (A) Uncontrolled } \\
\text { studies } \\
\text { Smith et al. (1992) }\end{array}$ & $\begin{array}{l}\text { CBT' } \\
\text { (16 sessions) }\end{array}$ & $8 \mathrm{~B}$ & $11 \%$ & $-1.1 \mathrm{~kg}$ & $\begin{array}{l}50 \% \text { abstinent } \\
81 \% \text { reduction }\end{array}$ & - & - & 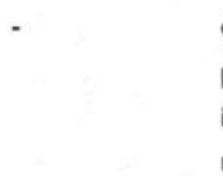 & $\begin{array}{l}\text { depression and eating } \\
\text { path. }\left(E^{2} E^{2}\right) \text { significant } \\
\text { improvement restraint } \\
\text { no improvement }\end{array}$ \\
\hline $\begin{array}{l}\text { (B) Controlled } \\
\text { (comparative) studies } \\
\text { Agras et al. (1994) } \\
\text { \& Agras et al. (1997) }\end{array}$ & $\begin{array}{l}\mathrm{TBT}^{3} \\
\text { (30 sessions) }\end{array}$ & $37 \mathrm{~B}$ & $27 \%$ & $\begin{array}{l}-2.0 \mathrm{~kg}^{4 /} \\
-3.7 \mathrm{~kg}^{5}\end{array}$ & $\begin{array}{l}44 \% \text { reduction }^{4 /} \\
19 \% \text { abstinent }^{5} \\
67 \% \text { reduction }\end{array}$ & 3 months & $-4.2 \mathrm{~kg}$ & $\begin{array}{l}14 \% \text { abstinent } \\
56 \% \text { reduction }\end{array}$ & $\begin{array}{l}\text { depression and } \\
\text { restraint } \\
\mathrm{TBT}=\mathrm{CBT} / \mathrm{TBT}= \\
\mathrm{CBT} / \mathrm{TBT}+\mathrm{D}\end{array}$ \\
\hline & $\begin{array}{l}\text { СВT/TBT } \\
(12+18 \text { sessions })\end{array}$ & $36 \mathrm{~B}$ & $17 \%$ & $\begin{array}{l}+0.7 \mathrm{~kg}^{4 /} \\
-1.6 \mathrm{~kg}^{5}\end{array}$ & $\begin{array}{l}67 \% \text { reduction } 4 / \\
37 \% \text { abstinent } 5 \\
73 \% \text { reduction }\end{array}$ & & $\begin{array}{l}0.0 \mathrm{~kg} \\
\text { (after I year) } \\
(+0.5 \mathrm{~kg})\end{array}$ & $\begin{array}{l}28 \% \text { abstinent } \\
61 \% \text { reduction } \\
\text { (after I year) } \\
\text { ( } 33 \% \text { abstinent) } \\
\text { (64\% reduction) }\end{array}$ & \\
\hline & $\begin{array}{l}\text { CBT/TBT+D } \\
(12+18 \text { sessions })\end{array}$ & $36 \mathrm{~B}$ & $23 \%$ & $\begin{array}{l}+0.7 \mathrm{~kg}^{4} / \\
-6.0 \mathrm{~kg}^{5}\end{array}$ & $\begin{array}{l}67 \% \text { reduction } 4 / \\
41 \% \text { abstinent } \\
5 \\
82 \% \text { reduction }\end{array}$ & & $-4.8 \mathrm{~kg}$ & $\begin{array}{l}32 \% \text { abstinent } \\
71 \% \text { reduction }\end{array}$ & \\
\hline
\end{tabular}




\section{Table 2.2 Continued}

\begin{tabular}{|c|c|c|c|c|c|c|c|c|c|}
\hline Author & $\begin{array}{l}\text { Treatment } \\
\text { Components }\end{array}$ & $\begin{array}{l}\text { Sample } \\
\text { size }\end{array}$ & $\begin{array}{l}\text { Drop- } \\
\text { outs }\end{array}$ & $\begin{array}{l}\text { Treatment } \\
\text { response } \\
\text { weight loss }\end{array}$ & $\begin{array}{l}\text { Treatment } \\
\text { response } \\
\text { bingeing }\end{array}$ & $\begin{array}{l}\text { Follow-Up } \\
\text { (FU) }\end{array}$ & $\begin{array}{l}\text { FU-response } \\
\text { weight loss }\end{array}$ & $\begin{array}{l}\text { FU-response } \\
\text { bingeing }\end{array}$ & $\begin{array}{l}\text { Other } \\
\text { psychological } \\
\text { measures }\end{array}$ \\
\hline \multirow[t]{2}{*}{$\begin{array}{l}\text { Agras } \\
\text { et al. (1995) }\end{array}$} & $\begin{array}{l}\text { CBT } \\
\text { (12 sessions) }\end{array}$ & $39 \mathrm{~B}$ & $14 \%$ & $+1.4 \mathrm{~kg}$ & $\begin{array}{l}55 \% \text { abstinent } \\
84 \% \text { reduction }\end{array}$ & $\cdot$ & - & - & $\begin{array}{l}\text { depression and } \\
\text { restraint } \\
C B T>W L^{8}\end{array}$ \\
\hline & Waiting list & II B & $9 \%$ & $+3.7 \mathrm{~kg}$ & $\begin{array}{l}9 \% \text { abstinent } \\
8 \% \text { reduction }\end{array}$ & & $\cdot$ & - & $\begin{array}{l}\text { self-esteem no } \\
\text { improvement }\end{array}$ \\
\hline & $\mathrm{CBT}^{7}$ & $13 \mathrm{~B} /$ & $?$ & $+4.7 \mathrm{~kg}$ & $58 \%$ reduction & & $\cdot$ & $\cdot$ & \\
\hline & IPT(12 sessions) & \multicolumn{3}{|c|}{ non-respon. } & & & & & \\
\hline & $\mathrm{CBT} 7$ & $17 \mathrm{~B} /$ & $?$ & $-4.8 \mathrm{~kg}$ & $95 \%$ reduction & & - & - & \\
\hline & TBT (12 sessions) & \multicolumn{2}{|c|}{ responders } & & & & & & \\
\hline Allen \& & CBT (Appetite & $15 B$ & $27 \%$ & - & $85 \%$ reduction & - & - & $\cdot$ & depression and appetite \\
\hline \multirow[t]{3}{*}{ Craighead (1999) } & Awareness Training) & & & & & & & & and self-efficacy \\
\hline & (8 sessions) & & & & & & & & $\mathrm{CBT}>\mathrm{WL}$ \\
\hline & Waiting list & $14 \mathrm{~B}$ & $36 \%$ & - & $25 \%$ increase & & - & - & \\
\hline
\end{tabular}


Table 2.2 Continued

\begin{tabular}{|c|c|c|c|c|c|c|c|c|c|}
\hline Author & $\begin{array}{l}\text { Treatment } \\
\text { Components }\end{array}$ & $\begin{array}{l}\text { Sample } \\
\text { size }\end{array}$ & $\begin{array}{l}\text { Drop- } \\
\text { outs }\end{array}$ & $\begin{array}{l}\text { Treatment } \\
\text { response } \\
\text { weight loss }\end{array}$ & $\begin{array}{l}\text { Treatment } \\
\text { response } \\
\text { bingeing }\end{array}$ & $\begin{array}{l}\text { Follow-Up } \\
\text { (FU) }\end{array}$ & $\begin{array}{l}\text { FU-response } \\
\text { weight loss }\end{array}$ & $\begin{array}{l}\text { FU-response } \\
\text { bingeing }\end{array}$ & $\begin{array}{l}\text { Other } \\
\text { psychological } \\
\text { measures }\end{array}$ \\
\hline Goodrick & TBT & $79 \mathrm{~B}$ & $15 \%$ & $-0.6 \mathrm{~kg}$ & $\cdot$ & 1-year & $+1.5 \mathrm{~kg}$ & $\cdot$ & no additional \\
\hline \multirow[t]{8}{*}{ et al. (1998) } & (24 sessions + & & $18 \%$ & & & & & & outcome measures \\
\hline & 26 sessions & & & & & & & & \\
\hline & maintenance) & & & & & & & & \\
\hline & CBT & $78 \mathrm{~B}$ & $17 \%$ & $+1.4 \mathrm{~kg}$ & - & & $+1.2 \mathrm{~kg}$ & $\cdot$ & \\
\hline & (24 sessions + & & $21 \%$ & & & & & & \\
\hline & 26 sessions & & & & & & & & \\
\hline & maintenance) & & & & & & & & \\
\hline & Waiting list & $62 \mathrm{~B}$ & $6 \%$ & $+0.6 \mathrm{~kg}$ & - & & - & - & \\
\hline \multirow{9}{*}{$\begin{array}{l}\text { Marcus } \\
\text { et al. (1995) }\end{array}$} & & $115 \mathrm{~B}$ & & & pre-treatment & 1-year10 & & & \\
\hline & & (total) & & & 21.6 binge days 9 & only 28 B & & & \\
\hline & & & & & & were & & & \\
\hline & Individual TBT & ? & ? & $-9.8 \mathrm{~kg}$ & 2.7 binge days & available & $?$ & $?$ & depression \\
\hline & (6 months) & & & & last 28 days & for FU & & & $\mathrm{TBT}+\mathrm{CBT}>\mathrm{WL}$ \\
\hline & Individual CBT & $?$ & ? & $0.0 \mathrm{~kg}$ & 0.7 binge days & & $?$ & $?$ & \\
\hline & (6 months) & & & & last 28 days & & & & \\
\hline & Waiting list & $?$ & ? & $0.0 \mathrm{~kg}$ & 18.1 binge days & & - & - & \\
\hline & & & & & last 28 days & & & & \\
\hline
\end{tabular}


Table 2.2 Continued

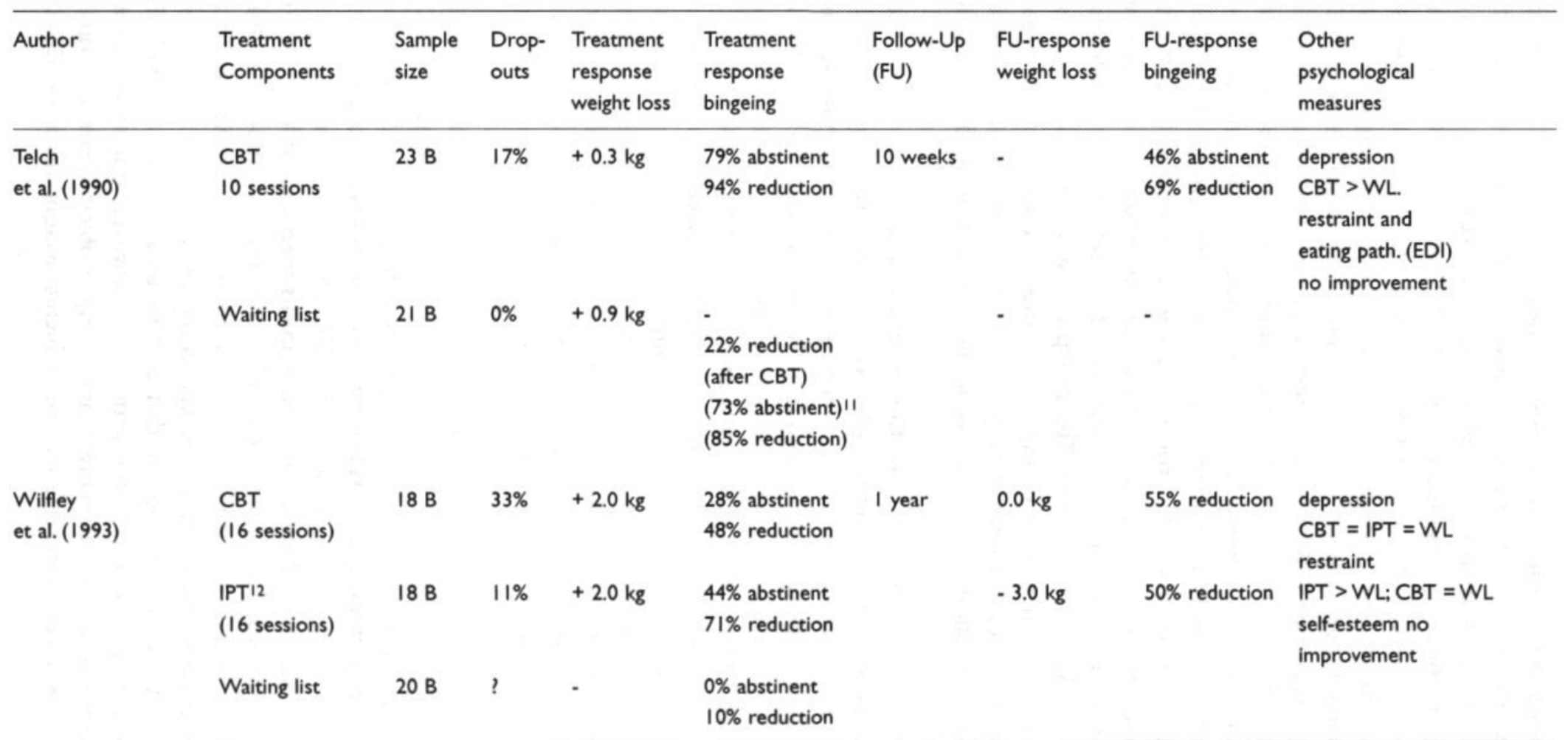

I CBT = Cognitive-Behavioral Therapy; ${ }^{2}$ EDE $=$ Eating Disorder Examination; ${ }^{3} \mathrm{TBT}=$ Traditional Behavior Therapy; ${ }^{4}$ Treatment results after 12 weeks; ${ }^{5}$ Treatment results after 30 sessions; ${ }^{6} \mathrm{D}$ = Desipramine; ${ }^{7}$ In this study all participants received first 12 weeks CBT. Thereafter, CBT non-responders received Interpersonal Therapy and treatment responders received TBT; ${ }^{8}$ WL = Waiting List; ${ }^{9}$ The mean binge scores at pretreatment of the separate treatment conditions were not mentioned; 10 Only 28 subjects of the 115 (24\% of the total sample) were available;

11 represents treatment response after waiting list subjects entered treatment; 12 IPT = InterPersonal Therapy 
Cognitive-behavioral therapy versus traditional behavioral treatment. Three different studies have compared the effects of CBT with those of TBT (Agras et al., 1994; Goodrick et al., 1998; Marcus et al., 1995). Agras and colleagues (1994) randomly allocated participants to either a 9-month TBT, a 3-month CBT followed by TBT for 6-month (CBT/TBT) or a CBT followed by TBT plus desipramine $(\mathrm{CBT} / \mathrm{TBT}+\mathrm{D})$. After the first 3 -months, CBT was superior to weight loss treatment in reducing binge eating ( $67 \%$ versus $44 \%)$, whereas the weight loss treatment was superior to CBT in weight reduction $(2.0 \mathrm{~kg}$ loss versus $0.7 \mathrm{~kg}$ gain). At posttreatment (after 9-months of treatment) the three groups did not differ significantly in binge frequency or weight loss. At 3-months follow-up there was, again, no significant difference among the three groups in frequency of binge eating. However, the $\mathrm{CBT}$ group receiving medication lost significantly more weight than the CBT group without medication. Finally, the results of a 1 -year follow-up were only described for the obese binge eating individuals treated with CBT (Agras et al., 1997). The group as a whole continued to show reasonable reductions in binge eating, and showed a slight weight gain. However, those who stopped binge eating during CBT maintained a weight loss of $4.0 \mathrm{~kg}$ during the follow-up period, while those who continued to binge eat gained $3.6 \mathrm{~kg}$.

In the second study conducted by Goodrick and colleagues (1998), 219 binge eating women were randomly assigned to a diet treatment, a non-diet treatment with cognitive elements or a waiting-list control. At posttreatment, participants in the diet treatment lost significantly more weight than participants in the non-diet treatment ( $0.6 \mathrm{~kg}$ weight loss versus $1.4 \mathrm{~kg}$ weight gain). However, neither TBT nor CBT was significantly different from the waiting list control group. At 1-year follow-up, both treatments achieved similar gains of more than $1 \mathrm{~kg}$ above baseline. The results indicate that neither intervention was successful in producing short-term or long-term weight loss. In this study, therapist biases (both groups were led by psychotherapist and spontaneous dis42 cussions took place about the role of obesity in our culture) might have affected treatment integrity, and these were discussed in relation to the small weight losses achieved.

Marcus and colleagues (1995) compared an individual CBT with an individual TBT and a delayed treatment group. After 6-month treatment, both CBT and TBT were equally effective in reducing binge eating frequency ( 0.7 binge days versus 2.7 binge days, respectively). However, participants in the TBT lost significantly more weight than those in the CBT $(9.8 \mathrm{~kg}$ versus $0.0 \mathrm{~kg})$. At long-term follow-up, only 28 of the 115 participants were available. Due to the great percentage of dropouts, the 1-year follow-up results cannot be clearly interpreted. Nevertheless, the data seems to indicate that participants in the weight loss treatment showed significant weight regain.

In conclusion, these studies suggest that at long-term follow-up, both TBT and $\mathrm{CBT}$ are equally efficacious. In the short-term, however, one study showed that $\mathrm{CBT}$ is more effective in reducing binge eating problems. Furthermore, in the short-term, TBT was more effective in reducing weight than the CBT. At 
long-term follow-up, CBT participants remained stable, while the participants in TBT gained weight. In addition, obese binge eaters who are abstinent from binge eating after treatment seem to lose weight at follow-up, while obese binge eaters who do not abstain from binge eating seem to gain weight at follow-up.

Cognitive-behavioral therapy versus InterPersonal Therapy. One study compared group CBT to group IPT (Wilfley et al., 1993). At posttreatment, both treatment conditions showed significant improvement in reducing binge eating. Both groups were significantly superior to the waiting list condition, but were not significantly different from each other. IPT participants reduced the frequency of binge eating by $71 \%$ with $44 \%$ of the participants reporting abstinence from binge eating. CBT participants reduced binge eating by $48 \%$ with a $28 \%$ abstinence rate. During the follow-up period, binge eating remained significantly below baseline levels for both treatment conditions. CBT and IPT were equally effective in reducing binge eating. At posttreatment and follow-up, participants in both treatment conditions did not differ significantly on weight loss. In sum, IPT is as effective as CBT and may be a useful alternative for those suffering from BED. However, further research is needed to replicate this study.

Treatment for cognitive-behavioral non-responders. Short-term studies of CBT for obese binge eaters indicate that about $50 \%$ of these do not abstain from binge eating after CBT. Agras and colleagues (1995) suggested that a second-level intervention was needed for poor responders to CBT. They evaluated the effectiveness of a 12-week IPT for CBT non-responders and found that IPT did not result in further improvement for CBT non-responders. Predictors of poor outcome were an early onset of, and more severe binge eating.

Eldredge and colleagues (1997) randomized 46 persons with BED to a CBT or to a waiting list control condition. Responders received an additional weight loss protocol, whereas non-responders were assigned to an additional 12 weeks of CBT. Of the initial non-responders, $43 \%$ no longer met the criteria for BED by the end of treatment. The results suggests that an initial course of CBT lasting more than 12 weeks may be desirable to maximize the number of potential responders to treatment. Definite conclusions about an intensive approach for non-responders require additional research.

\section{Cognitive-behavioral therapy for obese binge eaters and obese non-binge eaters}

Two studies have compared both obese binge eaters and obese non-binge eaters (Marcus et al., 1988; Porzelius, Houston, Smith, Arfken, \& Fisher, 1995). See also Table 2.3.

Marcus and colleagues (1988) compared a traditional behavioral therapy with a modified therapy incorporating a number of cognitive strategies. Binge eaters were more likely to drop out of treatment. No differences in weight loss emerged between binge eaters and non-binge eaters and between the standard and modified treatment. However, obese binge eaters had regained significantly 
Table 2.3 Studies of cognitive-behavioral therapy for obese binge eaters (B) and obese non-binge eaters (NB)

\begin{tabular}{|c|c|c|c|c|c|c|c|c|c|}
\hline Author & $\begin{array}{l}\text { Treatment } \\
\text { Components }\end{array}$ & $\begin{array}{l}\text { Sample } \\
\text { size }\end{array}$ & $\begin{array}{l}\text { Drop- } \\
\text { outs }\end{array}$ & $\begin{array}{l}\text { Treatment } \\
\text { response } \\
\text { weight loss }\end{array}$ & $\begin{array}{l}\text { Treatment } \\
\text { response } \\
\text { bingeing }\end{array}$ & $\begin{array}{l}\text { Follow-Up } \\
\text { (FU) }\end{array}$ & $\begin{array}{l}\text { FU-response } \\
\text { weight loss }\end{array}$ & $\begin{array}{l}\text { FU-response } \\
\text { bingeing }\end{array}$ & $\begin{array}{l}\text { Other } \\
\text { psychological } \\
\text { measures }\end{array}$ \\
\hline Marcus & TBTI & $17 \mathrm{NB}$ & $18 \%$ & $-4.9 \mathrm{~kg}$ & - & 6 months/ & $-5.2 \mathrm{~kg} /-2.9 \mathrm{~kg}$ & - & depression improvement \\
\hline \multirow[t]{4}{*}{ et al. (1988) } & ( 10 sessions) & $17 \mathrm{~B}$ & $24 \%$ & $-4.3 \mathrm{~kg}$ & - & I year & $-0.9 \mathrm{~kg} /-1.7 \mathrm{~kg}$ & - & $\mathrm{TBT}=\mathrm{CBT}$ \\
\hline & & & & & & & & - & restraint no \\
\hline & $\mathrm{CBT}^{2}$ & $16 \mathrm{NB}$ & $0 \%$ & $-5.4 \mathrm{~kg}$ & - & & $-4.4 \mathrm{~kg} /-2.4 \mathrm{~kg}$ & - & improvement \\
\hline & (10 sessions) & $18 \mathrm{~B}$ & $28 \%$ & $-5.0 \mathrm{~kg}$ & - & & $-2.8 \mathrm{~kg} /-1.6 \mathrm{~kg}$ & - & \\
\hline Porzelius & TBT & $8 \mathrm{NB}$ & $13 \%$ & $-8.5 \mathrm{~kg}^{4}$ & - & I year & $-2.5 \mathrm{~kg}$ & - & depression and restraint \\
\hline \multirow[t]{6}{*}{ et al. (1995) } & (15 sessions) & $8 \mathrm{MB}^{3}$ & $13 \%$ & $-5.0 \mathrm{~kg}$ & - & & $-2.0 \mathrm{~kg}$ & - & improvement \\
\hline & & $9 \mathrm{~B}$ & $22 \%$ & $-2.6 \mathrm{~kg}$ & - & & $+0.4 \mathrm{~kg}$ & - & $\mathrm{TBT}=\mathrm{CB} T$ \\
\hline & CBT (binge eating & $8 \mathrm{NB}$ & $13 \%$ & $-3.4 \mathrm{~kg}$ & - & & $-3.0 \mathrm{~kg}$ & - & \\
\hline & weight loss treatment & $10 \mathrm{MB}$ & $10 \%$ & $-0.2 \mathrm{~kg}$ & - & & $+1.8 \mathrm{~kg}$ & - & \\
\hline & + cognitive elements) & $11 \mathrm{~B}$ & $18 \%$ & $-5.0 \mathrm{~kg}$ & - & & $-8.0 \mathrm{~kg}$ & - & \\
\hline & (15 sessions) & & & & & & & & \\
\hline
\end{tabular}

I $\mathrm{TBT}=$ Traditional Behavior Therapy: ${ }^{2} \mathrm{CBT}=$ Cognitive-Behavioral Therapy: ${ }^{3} \mathrm{MB}=$ Mild Binge eater $;{ }^{4}$ Estimated from a figure. 
more weight than obese non-binge eaters at 6-months' follow-up. Reduction of binge eating episodes was not measured in this study.

In the second study, Porzelius and colleagues (1995) examined the effects of a TBT and a CBT modified to better address binge eating problems (e.g. greater emphasis on cognitions contributing to stressful situations and negative moods) for individuals with no, moderate, and severe binge eating problems. At 1-year follow-up, individuals with severe binge eating in the CBT had lost more weight than those in TBT ( $8.0 \mathrm{~kg}$ weight loss versus $0.4 \mathrm{~kg}$ weight gain). For obese non-binge eaters, however, weight loss did not differ between the two treatments at 1-year follow-up ( $3.0 \mathrm{~kg}$ weight loss versus $2.5 \mathrm{~kg}$ weight loss). The outcome measure binge eating was assessed with a self-report questionnaire, the Binge Eating Scale (Gormally et al., 1982). In both treatments binge eating was significantly reduced, and was not reduced more by the CBT.

The data from both studies require confirmation, because the diagnosis of binge eater or non-binge eater at pretreatment were based on the Binge Eating Scale and not on the DSM-IV criteria for BED. Furthermore, it could be argued that the treatments did not differ substantially. Finally, the drop-out rates among obese binge eaters were high.

\section{Conclusions}

All in all, CBT had positive effects for obese binge eaters and obese non-binge eaters. For obese non-binge eaters it seems that in the short-term TBT might be more promising than $\mathrm{CBT}$ in reducing weight. However, at long-term followup, no differences were found between CBT and TBT. Furthermore, an interesting result found in most studies is that CBT might have broader effects and could also improve several psychological variables such as body dissatisfaction, depression, and self-esteem. Although, CBT had positive effects on binge eating for obese binge eaters, no effect for weight loss was achieved. In addition, obese binge eaters who stopped binge eating lost more weight than obese binge eaters who continued binge eating. Furthermore, a number of studies found positive effects on shape and weight concerns and depression. Finally, Porzelius and colleagues (1995) found that at 1-year follow-up, individuals with severe binge eating in CBT lost more weight than those in TBT, whereas for obese non-binge eaters weight loss did not differ between the two treatments at 1-year follow-up.

This dissertation aims to examine which part of the cognitive-behavioral therapy is effective for obesity and binge eating disorder, the cognitive part or the non-dieting behavioral part? So far, these two components have never been examined separately. The cognitive therapy was expected to produce greater changes in psychological well-being (depression and self-esteem) and dysfunctional cognitions, although in the short term weight loss was expected to be greater in the behavioral therapy. 
CHAPTER 3

\section{TREATMENT PROTOCOLS AND OUTLINE OF THIS THESIS}

\section{DESCRIPTION OF THE TREATMENT PROTOCOLS}

A detailed description will be given of the cognitive and behavioral protocols

developed for the research mentioned in this thesis ${ }^{3}$.

\section{General aspects}

Both treatments were given in groups and all groups met for 15 weekly sessions of 150 minutes each. The groups, each consisting of 7 or 8 participants were assisted by one therapist. The therapists were experienced in the behavioral and cognitive therapy of eating disorders and/or obesity. The treatment protocols were based upon international treatment manuals outlined by Beck (1976) and Fairburn and colleagues (1993). 
For both conditions, detailed treatment protocols were developed. All therapists received a training in the protocol. Weekly consultation sessions of approximately one hour with each therapist were held to ensure therapists' compliance with treatment protocols. The therapists checked treatment compliance of participants. Absence or presence of participants was recorded every session and completion of homework assignments was checked.

Both treatments were semi-structured and problem oriented, were primarily concerned with the participants' present and future rather than past, provided a coherent treatment rationale, were self-monitoring, and set homework assignments. All sessions contained the following: (1) brief overview of the agenda, (2) review of homework, (3) active collaborative work on the agenda items, (4) group and therapists' feedback, (5) assignment of homework.

\section{Behavioral therapy}

The aim of the behavioral therapy was to learn a regular and healthy eating pattern. The first stage of therapy (session 1-7) focused on learning a regular and healthy eating pattern and on increasing the exercise level. In the first session, group members introduced themselves and reviewed their reasons for treatment and expectations for the group. The therapist introduced herself and considerable effort was taken to establish a positive collaborative alliance and an effective working relationship. Furthermore, details regarding the group structure were provided. Thereafter, the therapists explained the rationale of behavioral therapy. To regain control over eating it is important to learn a healthy eating pattern. Participants were told that treatment needed first to eliminate binge eating and overeating patterns by establishing a regular, healthy eating pattern and that weight control needed to be a secondary concern. A regular eating pattern was introduced with three regular meals and three planned snacks. They were stimulated to moderate overall intake in a range between 1500 and 1800 calories a day. Finally, the daily food record was explained. Participants were told to selfmonitor their food intake, binge episodes or episodes of overeating and the circumstances under which they occurred, such as time, presence of other people, and mood. Examination of the daily food records formed an important focus of therapy and this work was reviewed at the start of each session. In the first half of the second session homework assignments were discussed and in the second half, nutritional information was given. From now on nutrition education was included in every session. Participants were introduced to the basic principles of good nutrition. Specifically, participants were encouraged to decrease total fat intake and to increase consumption of complex carbohydrates and fiber. It was emphasized that such changes are consistent recommendations for all adults, in order to promote health. Moreover, the specific skills required to be able to eat healthy were taught, with lessons on topics such as label reading, special cooking skills and restaurant eating. Furthermore, participants were advised to make gradual changes in eating habits and exercise level. Therefore, weekly goals were 
set to change their eating habits and general activity levels. Therapists were instructed to reinforce participants if they reached the weekly goal. Session 3 through session 7 involved more detailed steps of how to create a healthy and regular eating pattern and a more active lifestyle.

In the second stage (session 8-13) stimulus control techniques were introduced. Three forms of stimulus control techniques were used. First, participants were taught stimulus control techniques whereby stimuli that resulted in overeating were avoided. Examples of this were: Not going to a shop when hungry, selecting an eating place, restricting eating to that place, separate eating from other activities such as watching television, and storing food out of sight. Second, they were taught techniques to anticipate high risk situations and to outline strategies to prevent eating, such as walking, talking with friends, or taking a shower. Third, participants were taught self-reinforcement techniques, such as going to the movies or buying a magazine, and were positively reinforced by the therapist if they were not overeating. No attention was paid to dysfunctional cognitions.

The final stage was largely concerned with the maintenance of progress following the end of treatment. The steps mentioned earlier in treatment were repeated. Participants were told that is important to keep a regular and healthy eating pattern to prevent overeating and binge eating in the future. In stressful situations they were stimulated to write down everything they ate, to plan their intake and to use the different stimulus control techniques they had learned in treatment.

\section{Cognitive therapy}

The aim of cognitive therapy was to change dysfunctional cognitions regarding shape, weight, eating, and dieting and underlying self-schemas with help of the cognitive techniques outlined by Beck (1976). Cognitive theory states that cognitions influence behavior in a predictable manner, and that cues, both internal and external, can provoke behavioral consequences through activation of cognitive sets (Beck, 1976). As such, effecting change in negative cognitions will influence behavioral outcome.

The first stage of the therapy (6 sessions), focused on identifying and altering dysfunctional cognitions regarding shape, weight, and eating. The Socratic dialogue was used by the therapist to initialize active learning and to encourage a questioning, investigative attitude in the participants. Furthermore, participants were encouraged to engage in behavioral experiments designed to challenge their dysfunctional cognitions. In the first session, the group members introduced themselves and reviewed their reasons for treatment and expectations for the group. The therapist introduced herself and considerable effort was taken to establish a positive collaborative alliance and an effective working relationship. Furthermore, details regarding the group structure were provided. For example, attendance was essential and the treatment requires full commitment 
and must represent a priority in person's life. Thereafter, the therapist explained the rationale of the cognitive therapy. A binge/overeating circle was described as beginning with dysfunctional cognitions about shape, weight, eating, dieting or a negative self-schemas. The daily thought record was explained. The therapist explicitly stated that she would give homework each week, an important focus of the therapy, and that this work would be reviewed at the start of each session. The initial homework assignment for the participants was to self-monitor situations that triggered binge eating or overeating and to identify their thoughts. At the end of all sessions there was a short evaluation of the session. In the second session all participants were asked to review their self-monitoring form. If participants did not complete homework, it was determined what prevented them from doing the homework. The therapist reinforced the efforts of the participants as well as the interactive aspects of the group. After identifying the dysfunctional cognitions of the participants, during the second part of the session several categories of faulty thinking were mentioned, such as overgeneralization, selective abstraction, catastrophizing, and dichotomous thinking. Sessions 3 through 6 involved more detailed discussion of how to challenge and alter dysfunctional cognitions about shape, weight, and eating. The group was supposed to gradually become a forum for learning how to be increasingly aware of the occurrence of dysfunctional cognitions regarding eating, weight, and shape, for addressing them, and for generating possible alternatives. Many obese persons did report negative feelings towards their own body. Not for all obese persons it was possible to fully accept their own body. The therapist should not suggest that they must like their whole body. Acceptance of even small parts of their body was enough. Although, they had to know that selfcontempt only served to increased feelings of desperation and that as a consequence overeating or binge eating was more likely to occur. During session 6 behavioral experiments were designed to test their dysfunctional cognitions regarding shape, weight, or eating. Behavioral experiments directly tested the validity of a person's thoughts or assumptions.

In the second stage (sessions 7-12), cognitive tech-niques directed at identify-ing and challenging negative self-schemas that perpetuate disordered eating were emphasized. Negative self-schemas were identified and challenged and also behavioral experiments were used. In session 7 underlying self-schemas of the participants were identified. Underlying schemas were discovered by the 'downward arrow' technique, in which the consequences of a particular thought were repeatedly drawn out. In this way the focus can shift from thoughts about specific situations to more general concerns, e.g. 'I am worthless', 'I have no willpower'. Helping participants set reasonable goals during this phase was essential, total acceptance of themselves was an unlikely outcome. Dysfunctional thoughts reported by individuals with obesity were also related to social stereotypes about obesity (Crandall, 1994). Typical beliefs were that fat people lack discipline or that obese persons were disgusting. It was important to make prejudice explicit and to help obese persons to deal with these negative societal attitudes. Rather than accepting criticism as being per- 
sonal relevant, obese persons should recognize it as prejudice. Moreover, because most obese persons blame themselves excessively for their overweight, it was important that they realized that obesity has also a strong genetic component. Session 8 through session 11 involved more detailed discussion of how to challenge and alter negative self-schemas and to build a more positive self esteem. Participants also designed behavioral experiments. For example, participants were stimulated to go to the swimming pool or the sauna. In these experiments they were discouraged from only comparing themselves to people who are thinner then they.

The final stage (sessions 13-15) was largely concerned with the maintenance of progress following the end of treatment. A lapse usually becomes a relapse as a result of underlying all-or-none beliefs; for example, 'A lapse means, I don't have any control', or 'A lapse is a failure'. Marlatt and Gordon (1985) call this thinking process and the resulting relapse the Abstinence Violation Effect (AVE). The AVE accounts for some of the variability regarding relapse. The AVE model predicts that internal, stable and global attributions for a lapse increase the probability of overeating or binge eating. The purpose of the cognitive restructuring procedure in the last phase was to reframe maladaptive attributions of causality regarding lapses, to make it easier to regain control. Participants were stimulated to mention difficulties in the future, especially at times of extreme stress. Participants were informed that lapses were normal and to be expected and that the participant should construct a plan for these situations.

\section{OVERVIEW OF THE DISSERTATION}

The aim of this dissertation is to examine the short- and long-term effectiveness of cognitive therapy versus behavioral therapy for obese binge eaters and obese non-binge eaters.

Before that, Chapter 4 will present a study aimed at examining the frequency and content of several dysfunctional cognitions in obese binge eaters and obese non-binge eaters. A structured clinical interview was used to detect cognitive differences between these two groups. Obese participants were asked to report the thoughts that had run through their minds in situations in which they were struggling with their shape, weight, or eating during the last four weeks. If participants mentioned dysfunctional thoughts regarding shape, weight, and eating, interviewers explored the underlying schemas by using the 'downward arrow' technique. Implications for future work are discussed, including how cognitive techniques focusing on dysfunctional cognitions might improve treatment for obesity.

Chapter 5 presents a study which aims to evaluate the short-term effectiveness of a cognitive and a behavioral therapy for obese binge eaters and obese 
non-binge eaters. Seventy-four obese participants, 37 of them binge eaters and 37 non-binge eaters, were randomly assigned to one of the two treatment conditions, taking the binge status into account. Both treatments took place in groups and all groups met for 15 weekly sessions of 150 minutes each. Participants in the cognitive therapy received instruction about changing dysfunctional cognitions, and participants in the behavioral therapy about a regular eating pattern.

Chapter 6 presents the long-term results of the cognitive and behavioral therapy. Furthermore, special attention is paid to the role of weight changes on psychological well-being at long-term follow-up. Participants were reassessed 6month and 1-year upon completion of the cognitive therapy or the behavioral therapy.

Chapter 7 tries to identify which pretreatment patient characteristics predict 1-year outcome following cognitive or behavioral therapy for obesity. Additionally, in this Chapter the central prediction of the cognitive model of obesity is tested, i.e., that participants' shape and weight concerns at posttreatment are inversely related to treatment success.

Chapter 8 includes a general discussion of the studies. It summarizes and discusses the major findings of the studies. Furthermore, recommendations for clinical practice and future research are given. The reader will notice some repetition in the following Chapters, particularly in the method section. This is due to the fact that the empirical studies are primarily a collection of published articles, which required each Chapter to be read as a self-contained entity. 


\section{COGNITIONS IN OBESE BINGE EATERS AND OBESE NON-BINGE EATERS}

\section{Published as:}

Nauta, H., Hospers, H.J., Jansen, A., \& Kok, G. (2000). Cognitions in obese binge eaters and obese non-binge eaters. Cognitive Therapy and Research, 24, 521-531.

\section{INTRODUCTION}

Cognitions such as preoccupation with food and eating and cognitive distortions regarding body image and weight play a central role in the progression and maintenance of eating disorders (Cooper \& Fairburn, 1992; Dritschel, Williams, \& Cooper, 1991; Garner \& Bemis, 1982). In contrast to eating disorders, cognitions about shape, weight, and eating have figured less prominently in etiological theories of obesity. However, some studies have been conducted to examine relationships between cognitive content and the development and maintenance of obesity (Adami et al., 1994; Garner, Olmsted, \& Polivy, 1983; Hunt \& Rosen, 1981; O'Connor \& Dowrick, 1987; Phelan, 1987; Sunday, Halmi, Werdann, \& Levey, 1992).

In the assessment of cognitions in obesity, two general research methodologies have been used: in vivo data collection and retrospective self-statement questionnaires. Hunt and Rosen (1981) used a random time-sampling procedure and collected self-monitored cognitions from normal-weight and obese females. No group differences were found with respect to the frequency and quality of food, eating, and body-image related thoughts. O'Connor and Dowrick (1987) asked 
normal-weight, obese, and previously obese people to rate belief in and frequency of 26 dysfunctional cognitions concerning weight, food and eating. For example 'I have absolutely no self-control when it comes to food'. Obese participants reported higher belief in dysfunctional food and weight-focused cognitions than normal-weight people. In other studies, self-statement questionnaires designed for eating disorders have been administered to obese subjects. Adami et al. (1994) found that all scores of the Eating Disorder Inventory (EDI; Garner et al., 1983) were more elevated for obese persons than for normal-weight subjects. Other studies showed that obese subjects had a less pathological EDI profile than eating disordered subjects (Garner et al., 1983; Sunday et al., 1992). One study (Phelan, 1987), that used the Bulimic Thoughts Questionnaire found that obese subjects and eating disordered subjects scored similarly on cognitions about their ability to maintain a desirable weight. However, eating disordered subjects scored higher on cognitions about the unrealistic expectations of what would happen if 'forbidden food' was consumed, as well as about being out of control with food. In sum, the studies mentioned suggest that obese persons hold more dysfunctional cognitions than normal-weight persons, but have less pathologic scores on several measures of cognitions related to weight, shape, food, and eating than eating disordered subjects.

In recent years, there has been an increased interest in the exploration of binge eaters as a relatively homogeneous subgroup among the obese. Many studies have found that obese binge eaters experience more severe depression, have lower self-esteem (e.g., Marcus et al., 1990; Mitchell \& Mussell, 1995; Telch \& Agras, 1994; Yanovski, Nelson, Dubbert, \& Spitzer, 1993), and have more severe eating pathology than obese non-binge eaters (e.g., De Zwaan et al., 1995; Eldredge \& Agras, 1996; Fichter et al., 1993; Kuehnel \& Wadden, 1994; Wilson, Nonas, \& Rosenblum, 1993). Wilson et al. (1993) investigated differences in cognitive statements related to weight, shape, and eating between obese binge eaters and obese non-binge eaters, using a self-report version of 54 the Eating Disorder Examination (EDE-Q; Fairburn \& Beglin, 1994). When obese binge eaters were compared to obese non-binge eaters, they scored significantly higher in shape concern, weight concern and eating concern. Another study (Eldredge \& Agras, 1996) found that obese binge eaters scored significantly higher on the weight and shape subscales of the EDE-Q than obese nonbinge eaters, regardless of weight. Marcus, Smith, Santelli, and Kaye (1992) reported that there were no significant differences between obese binge eaters and bulimia nervosa patients on the shape, weight, and eating subscales of the EDE, except on restraint.

Vitousek and Hollon (1990) suggested that cognitive schemas that include cognitive generalizations about the self and that are derived from past experiences with weight, shape, and eating concerns constitute the core cognitive structures of eating disorders. Self-schemas are cognitive generalizations about the self, derived from past experience, that organize and guide the processing of the self-related information contained in an individual's social experience (Markus, 1977). A person who is schematic for some dimension processes 
information relevant to that dimension differently than someone who is aschematic for the dimension (Markus \& Sentis, 1982). Schematic subjects are more resistant to information that conflicts with their view of themselves, and are more likely to recognize schema-relevant than schema-irrelevant material. In an experimental design in normal-weight, overweight, and obese individuals, Markus, Hamill, and Sentis (1987) investigated self-schemas pertaining to body weight for their effects on methods of processing weight-relevant information. They found that the objective weight status of an individual did not correspond to method of processing weight-relevant information, but did correspond to the intensity in with which an individual is concerned about body weight and about a disposition to characterize weight as an organizing feature of the self (schematics). Beck, Wright, Newman, and Liese (1993) proposed that many persons are unable to articulate underlying self-schemas until they have been asked to consider the personal meaning that their shape, weight, and eating related cognitions have for them. In this way, self-schemas can be elucidated that are characterized as negative self-evaluations influenced by weight, shape, or eating. For example, a negative self-schema seen in anorexics and bulimics is: 'If I gain weight, I'm nothing' (Cooper, Cohen-Tovée, Todd, Wells, \& Tovée, 1997).

In this study, a structured clinical interview was used in which obese binge eaters and obese non-binge eaters were asked to mention cognitions related to shape, weight, and eating. Furthermore, underlying shape, weight, and eating related negative self-schemas were explored. The aim of the present study was to examine, in depth, the content and number of negative self-schemas, as well as weight, shape, and eating focused cognitions in obese binge eaters and obese non-binge eaters.

\section{METHOD}

\section{Subjects}

Selected participants were 74 obese women, including 37 women who met the proposed DSM-IV (APA, 1994) criteria for binge eating disorder (BED) and 37 non-binge eaters. They were selected from a group of respondents who answered local newspaper advertisements offering a university-based treatment for eating problems. Respondents received the Questionnaire on Eating and Weight Patterns-revised (QEWP-r; Yanovski, 1993). The QEWP-r is a self-report questionnaire designed to collect information regarding demographics, weight history, and eating patterns. The questionnaire also contains items that specifically test for DSM-IV criteria of BED. After completion at home, the questionnaires were returned to the university. 
If respondents met the BED criteria or did not have any binge episodes, they were invited for a structured diagnostic interview that lasted about 1-hour and was conducted by a psychologist experienced in the assessment and treatment of eating disorders and obesity. The interviewer clarified and checked the DSMIV items, especially those regarding quantity of food and extent of loss of control. The BED diagnosis was made if participants met the following criteria. First, respondents had to mention binge-eating episodes. Binge eating was operationalized as eating an objectively large amount of food and a feeling that the eating was out of control (see Fairburn, 1987). Binge eating had to occur at least 2 days per week during the previous 6-months, and binge-eating episodes had to be associated with three or more behavioral indicators of loss of control - for example, eating much more rapidly than normal, eating until feeling uncomfortably full, or eating large amounts of food when not physically hungry (APA, 1994). Finally, respondents had to report marked distress regarding binge eating. After the diagnostic interview, participants were weighed in street clothes, without shoes, on a balance-beam scale and their height was measured.

Respondents were categorized as non-binge eaters if they fulfilled none of the criteria mentioned. We anticipated that comparisons of these two extreme groups would be the most likely to reveal relevant differences. Therefore, respondents who met some but not all were excluded. Further exclusion criteria were: age younger than 18 or older than 50 years; concurrent treatment for weight loss; pregnancy; Body Mass Index (BMI) below 27; or concurrent DSMIV diagnosis of psychosis, drug abuse or alcoholism.

Participants who entered the study were between 21 and 49 years $(\mathrm{M}=$ $38.3, \mathrm{SD}=7.1)$. Their BMI ranged from 27.0 to $45.2(\mathrm{M}=33.4, \mathrm{SD}=4.2)$. Participants reported a mean duration of obesity of 21.1 years $(\mathrm{SD}=8.5)$ and a mean onset of obesity of 17.2 years $(S D=7.6)$. Binge eaters and non-binge eaters were compared on these variables using t-tests with Bonferroni correction 56 for multiple testing. The results showed that obese binge eaters were significantly younger and had an earlier onset of obesity, whereas group differences in BMI and duration of obesity were not significant. BED participants reported binge eating on an average of 4 days per week (SD = 1.7), and an onset of binge eating at a mean age of $21.4(\mathrm{SD}=6.2)$.

\section{Procedure}

Participants were invited to participate in a semi-structured face-to-face interview. The interview took between 30 and 45 minutes. All three interviewers had experience in measuring cognitions and working clinically with anorexic, bulimic, and/or obese patients. The interviewers were unaware of the clinical diagnosis of the participants. Participants were told that the purpose of the interview was to detect thoughts regarding shape, weight, and eating. All obese women were asked to report the thoughts that had run through their minds in 
situations in which they were struggling with their shape, weight, or eating during the 4-weeks preceding assessment. Most participants could 'automatically' report several dysfunctional thoughts. If participants had difficulties reporting thoughts, several situations that were likely to trigger shape, weight, and eating related cognitions, were presented to the participants. A checklist of these situations, taken from the literature, was constructed beforehand. Examples of such situations were: before or after weighing, when looking at your own body in a mirror, when others see your body at the swimming pool, when eating with other people, or when buying new clothes.

If people mentioned dysfunctional thoughts regarding shape, weight, and eating, interviewers explored the underlying schemas by using the "downward arrow' technique (Beck et al., 1993). At each belief, this technique points an arrow downward to the next underlying belief. Participants were asked what a cognition meant to them and what catastrophe could occur, with questions such as: 'If that cognition is true, what does it signify for you?' or 'If that cognition is true, what could possibly happen?' Sometimes, the result of the question was the elicitation of an underlying negative self-schema, formulated as an 'if ... then ...' statement. The conditional assumption ('if ...') stipulates the conditions under which the negative self-evaluation is applicable and, thus, becomes operative (Beck, 1996). If a participant did not mention a negative self-evaluation, they usually adhered to their more superficial thoughts - for example - that they were fat or could not control eating.

After dysfunctional cognitions had been identified, participants rated their degree of belief in these cognitions on Visual Analogue Scales (VASs), from 0 , 'totally disbelieved in', to 100, 'totally believed in'. The interviewers wrote down all cognitions on a whiteboard together with the corresponding degree of belief. If more than three cognitions were mentioned, the three cognitions believed in the most were selected for data analyses.

Finally, participants were given a number of questionnaires, including the Beck Depression Inventory (BDI; Beck, Ward, Mendelson, Mock, \& Erbaugh, 1961), the Rosenberg Self-Esteem Scale (Rosenberg, 1965), and three subscales (weight concern, shape concern, and eating concern) of the Eating Disorder Examination-Questionnaire (EDE-Q), a self-report version of the EDE (Fairburn \& Beglin, 1994).

\section{Classification of cognitions}

Three independent raters, all therapists in clinical practice and experienced in the treatment of eating disorders and obesity, were asked to categorize all cognitions ( $\mathrm{N}$ = 222). In Beck's (1976) model, dysfunctional cognitive content is divided into automatic thoughts and underlying schemas. In this study three categories were used: Negative Self-Schemas, Automatic Thoughts (weight/ shape concerns and control over eating), and Do Not Know. Self-schemas were defined as deeper cognitive structures that contain generalizations about the self 
combined with eating, weight, or shape concerns (e.g., 'If I eat too much, I am good for nothing'). Thus, negative self-schemas refer to negative self-evaluations. Automatic thoughts were defined as those ruminations or images that are typically experienced by the individual as occurring in their ongoing stream of thoughts regarding their shape and weight or loss of control over eating (e.g., 'If I have eaten too much, I can't stop eating any more'). The raters completed their task independently and were unaware of binge condition (binge eater/non-binge eater). They followed standardized instructions that included definitions, descriptions, and examples of negative self-schemas and automatic thoughts.

Interrater reliability was calculated by means of Cohen's kappa, and the average interrater reliability among the three raters was high (.82). Of the 222 cognitions, 31 were dropped because there was not $100 \%$ agreement among the raters. Furthermore, all five cognitions in the 'Do Not Know' category were excluded as well. Thus, 186 cognitions were included in further analyses.

\section{RESULTS}

\section{Amount of negative self-schemas and belief in cognitions}

Of the 96 cognitions mentioned by obese binge eaters, $64.6 \%$ (62) were negative self-schemas and $35.4 \%$ (34) were automatic thoughts. Approximately the reverse was found among non-binge eaters: negative self-schemas comprised $25.6 \%$ (23) of all cognitions, automatic thoughts $74.4 \%$ (67). This difference in distribution was highly significant $\_2(1)=28.5, \mathrm{p}<.001$.

A 2 (binge category: binge eater/ non-binge eater) $\times 2$ (cognitions: selfschema/ automatic thought) analysis of variance (ANOVA) was used to test for differences in degree of belief. There was a main effect for binge category, $\mathrm{F}(1,183)=36.0, \mathrm{p}<.001$, showing higher belief in cognitions for obese binge eaters $(M=90.3)$ than for obese non-binge eaters $(M=80.0)$. The difference in degree of belief for negative self-schemas versus automatic thoughts was not significant, $\mathrm{F}(1,183)=.08$, NS.

\section{Content of negative self-schemas}

In order to determine the thought content of negative self-schemas and automatic thoughts, a content analysis was conducted by researchers who were blind to binge category. Table 4.1 gives an overview of the classification of negative self-schemas. Of all negative self-schemas, $72 \%$ were related to lack of willpower, rejection by others, and unworthiness. Interestingly, negative self- 
Table 4.I Themes and examples of negative self-schemas in obese binge eaters and obese non-binge eaters

Themes

Examples

Schemas

Schemas

binge eaters non-binge eaters

$(\mathrm{N}=62)$

( $N=23$ )

\begin{tabular}{|c|c|c|c|}
\hline Weakness/ & 'If I eat forbidden food, I am a weakling' & & \\
\hline No willpower & 'If I gain weight, I am a person without willpower' & $22.6 \%$ & $43.5 \%$ \\
\hline Rejection & 'If I go to the swimming pool, people will reject me' & $30.6 \%$ & $8.7 \%$ \\
\hline Unwortiness & 'If I eat too much, I am good for nothing' & $22.6 \%$ & $8.7 \%$ \\
\hline Disgusting & 'If I look in the mirror, I see a disgusting person' & $6.5 \%$ & $4.3 \%$ \\
\hline Proof one's worth & 'Because I am fat, I always have to proof my worth' & $1.6 \%$ & $17.5 \%$ \\
\hline Abnormal & 'If I gain weight, I am physically and mencally abnormal' & $3.2 \%$ & $8.7 \%$ \\
\hline Alone & 'Without food, I am all alone' & $6.5 \%$ & $0.0 \%$ \\
\hline Vulnerable & $\begin{array}{l}\text { 'Only if my hips are narrow, I am not vulnerable } \\
\text { to criticism' }\end{array}$ & $1.6 \%$ & $4.3 \%$ \\
\hline Others & & $4.8 \%$ & $4.3 \%$ \\
\hline
\end{tabular}

\section{Others}

(2)




\section{Schematics versus Aschematics}

The focus of the analysis now shifts from cognitions $(\mathrm{N}=186)$ to respondents $(\mathrm{N}=73)$. One individual had no cognitions for which there was a $100 \%$ agreement between the raters and was dropped from the analyses. Three individuals had one remaining cognition, 27 individuals had two remaining cognitions, and 43 individuals had three remaining cognitions. If half or more of a participant's remaining cognitions were negative self-schemas, the respondent was regarded as schematic. If half or more of a participant's cognitions were automatic thoughts, the respondent was regarded as aschematic. A MANCOVA was conducted on eating pathology measures (shape concern, weight concern, eating concern) and general psychopathology (depression, self-esteem) with two between-subjects factors: binge category (binge eater/non-binge eater) and schema category (schematic/aschematic), while controlling for degree of belief. Results of the MANCOVA4 indicated a multivariate main effect for binge category, $\mathrm{F}(5,65)=18.8, \mathrm{p}<.001$. All univariate $\mathrm{F}$-tests revealed significant results: depression, $F(1,69)=22.9, \mathrm{p}<.001$, self-esteem, $\mathrm{F}(1,69)=14.7, \mathrm{p}<.001$, eating concern, $\mathrm{F}(1,69)=64.4, \mathrm{p}<.001$, shape concern, $\mathrm{F}(1,69)=70.2$, $\mathrm{p}<.001$, and weight concern, $\mathrm{F}(1,69)=29.6, \mathrm{p}<.001$, reflecting more general psychopathology and more eating pathology for obese binge eaters than obese non-binge eaters. Furthermore, a significant multivariate main effect for schemas was found $F(5,65)=2.9, \mathrm{p}<.05$. Univariate $\mathrm{F}$ tests revealed significant effects for depression, $\mathrm{F}(1,69)=6.3, \mathrm{p}<.05$ and self-esteem, $\mathrm{F}(1,69)=4.5$, $\mathrm{p}<.05$. Irrespective of binge category, schematic subjects were more depressed and had lower self-esteem than aschematic subjects. Schema category had no significant influence on any of the three subscale of the EDE. There was no significant interaction effect for schema category and binge category. Table 4.2 provides means and standard deviations for binge eaters and non-binge eaters with and without schemas on depression, self-esteem, and eating pathology.

Combining the finding that negative self-schemas were most frequently found in obese binge eaters with the observation that obese binge eaters were significantly more depressed than obese non-binge eaters, the question was raised whether the relationship between negative self-schemas and binge status might be carried by depression. It is not unlikely that depression influenced the accessibility of these negative self-schemas and that these negative self-schemas were thus more easily detected in the obese binge eaters. To explore this issue further, a logistic regression on schema category was conducted with binge status and depression as predictors. Both binge status and depression significantly contributed to the prediction of schema category. 
Table 4.2 Means and standard deviations for binge eaters and non-binge eaters with and without negative self-schemas on measures of generic psychopathology and eating pathology

\begin{tabular}{|c|c|c|c|c|c|c|c|c|}
\hline & \multicolumn{4}{|c|}{ Obese binge eaters } & \multicolumn{4}{|c|}{ Obese non-binge eaters } \\
\hline & \multicolumn{2}{|c|}{ Schematics } & \multicolumn{2}{|c|}{ Aschematics } & \multicolumn{2}{|c|}{ Schematics } & \multicolumn{2}{|c|}{ Aschematics } \\
\hline & Mean & (SD) & Mean & (SD) & Mean & (SD) & Mean & (SD) \\
\hline BDII & 19.9 & (7.8) & 17.1 & $(5.1)$ & 11.6 & $(4.1)$ & 6.7 & (4.7) \\
\hline RSES2 & 28.4 & (4.6) & 25.4 & $(5.0)$ & 22.6 & (3.7) & 19.9 & (5.3) \\
\hline EDE-Q eating concern ${ }^{3}$ & 3.3 & $(1.0)$ & 3.9 & (1.2) & 1.8 & $(0.7)$ & 1.1 & $(0.5)$ \\
\hline EDE-Q shape concern & 5.0 & $(0.6)$ & 5.3 & $(0.3)$ & 3.0 & (1.1) & 3.4 & $(0.9)$ \\
\hline EDE-Q weight concern & 4.1 & $(0.6)$ & 4.0 & $(0.6)$ & 2.8 & $(0.9)$ & 2.8 & $(0.8)$ \\
\hline
\end{tabular}

$1 \mathrm{BDI}=$ Beck Depression Inventory, ${ }^{2}$ RSE $=$ Rosenberg Self-Esteem Scale; ${ }^{3}$ EDE-Q $=$ Eating Disorder ExaminationQuestionnaire

\section{DISCUSSION}

This study found evidence that obese binge eaters and obese non-binge eaters differed with respect to the three cognitions in which they most believed, as detected by a clinical interview. The findings showed that obese binge eaters mentioned more self-schemas regarding negative self-evaluations than did obese non-binge eaters. Obese binge eaters believed their negative self-schemas and automatic thoughts to a greater extent than obese non-binge eaters. Moreover, obese binge eaters differed in the content of their negative self-schemas as compared to obese non-binge eaters. Finally, schematic participants reported more depression and lower self-esteem than aschematic participants.

A robust finding was that negative self-schemas, which reflect negative selfevaluations influenced by shape, weight, or eating, were more frequently found in obese binge eaters than in obese non-binge eaters. Considering the obese binge eaters as more eating disordered than the obese non-binge eaters, this finding supports Vitousek and Hollon (1990) who postulated that eating disordered individuals develop organized cognitive structures that unite views of the self with beliefs about weight, shape, and eating. It could be argued that the relationship between negative self-schemas and binge status might be carried by depression. Depression can influence the accessibility of negative self-schemas. However, a logistic regression on schema category revealed that both binge status and depression contributed significantly to the prediction of schema category. Depression, binge status, and schemas are related, without giving an answer to possible underlying causal structures. 
The content analysis of the negative self-schemas showed that schemas of binge eaters and non-binge eaters differed qualitatively. Obese binge eaters were more worried about rejection and unworthiness, whereas the non-binge eaters mainly worried about lack of willpower. Cognitions regarding rejection are associated with low self-esteem (Leary, Schreindorfer, \& Haupt, 1995), whereas cognitions of unworthiness and lack of willpower can be related to personal loss and failure, which are highly associated with depression (e.g., Clark \& Steer, 1996).

The data suggest that cognitive therapy might be useful in the treatment of obesity for binge eaters as well as non-binge eaters. Apart from challenging dysfunctional thoughts about weight, shape, and eating, it is necessary to address negative self-schemas. In case of obese binge eaters, one should be mindful of negative self-schemas regarding rejection and unworthiness, whereas in case of obese non-binge eaters negative self-schemas concerning lack of willpower might be relevant. Obese binge eaters believed their negative selfschemas even to a greater extent, and it is known that changes in affect are related to degree of belief in negative cognitions (Clark, 1988). Schema-focused techniques, such as those introduced by Young (1990), may be useful; these include the 'life review' technique, in which patients are asked to provide evidence from their lives that contradicts and supports their schemas, and 'schema flashcards', index cards that incorporate the evidence against and for the schemas.

The present study shows that obese subjects are characterized by dysfunctional cognitions concerning eating, shape and weight. It is assumed that obese individuals develop negative self-schemas in which views of the self are combined with beliefs about weight, shape, and eating. The negative self-schemas were highly accessible, especially in the binge-eating subgroup, presumably because binge eaters were more depressed and had lower self-esteem. Binge eaters reported more negative self-schemas of rejection and unworthiness,

62 whereas non-binge eaters mainly worried about lack of willpower. These findings are a step toward the clearer elucidation of dysfunctional cognitions in obese persons and suggest that the obese binge eaters as well as obese nonbinge eaters might benefit from cognitive therapy. 


\section{A COMPARISON BETWEEN A COGNITIVE AND A BEHAVIORAL THERAPY FOR OBESE BINGE EATERS AND OBESE NON-BINGE EATERS}

This section is a slightly adjusted version of the article: Nauta, H., Hospers, H., Kok, G., \& Jansen, A. (2000). A comparison between a cognitive and behavioral treatment for obese binge eaters and obese non-binge eaters. Behavior Therapy, 3I, 44I-46I.

\section{INTRODUCTION}

For more than 30 years behavioral methods have been systematically applied to the treatment of obesity. Controlled evaluation outcome research has demonstrated that the short- term effects of behavioral therapy are encouraging. The average weight loss is approximately 0.5 kilogram $(\mathrm{kg})$ per week (Wadden \& Bartlett, 1992). Despite encouraging results in the short-term, there remains a continuing failure to achieve long-term maintenance of weight: After 1-year almost all participants return to their baseline weights, regardless of the duration or method of therapy (Perri, Nezu, Patti, \& McCann, 1989; Wilson, 1994).

Besides the fact that behavioral therapy for obesity seems not to be very effective in producing lasting weight loss, several researchers have found that dieting itself has negative effects as well. Dieting might be associated with increased cardiovascular and all-cause mortality (Blair, Shaten, Brownell, Collins, \& Lissner, 1993; Lissner et al., 1991) and may result in pathological changes in cognition and affect (Brownell \& Rodin, 1994; Foreyt et al., 1995; Friedman \& Brownell, 1995; Polivy \& Herman, 1992). Moreover, there is evidence that strict dieting might be harmful and contributes to binge eating. In a prospective 
study of Telch and Agras (1993), it was found that $50 \%$ of obese women identified at baseline as non-binge eaters reported binge eating episodes 3 and 6months following a very low calorie diet, and $62 \%$ reported binge episodes at termination of the entire treatment program.

The cultural pressure to be thin is particularly intense for women (Horm \& Anderson, 1993), leading them to become overconcerned with their body shape and weight and to a low self-esteem (Friedman \& Brownell, 1995; Miller \& Downey, 1999; Stunkard \& Wadden, 1992). Obese individuals presenting for treatment of obesity do display a higher prevalence of psychological problems than obese individuals not seeking treatment (Friedman \& Brownell, 1995; Miller \& Downey, 1999). An important development in the study of obesity is the recognition that between $20 \%$ and $46 \%$ of obese patients seeking treatment report moderate to severe problems with binge eating (Bruce \& Wilfley, 1996; Gormally, Black, Daston, \& Rardin, 1982; Marcus, Wing, \& Lamparski, 1985; Spitzer et al., 1992; Telch, Agras, \& Rossiter, 1988). This subgroup of obese persons do display even more severe psychological problems than obese nonbinge eaters. Obese binge eaters show more concerns about shape, weight and eating than obese non-binge eaters (Eldredge \& Agras, 1996; Telch \& Stice, 1998; Wilson, Nonas, \& Rosenblum, 1993) at levels that are comparable to those mentioned by bulimia nervosa patients (Marcus, Smith, Santelli, \& Kaye, 1992). The level of restraint is not elevated and is comparable to obese nonbinge eaters. Furthermore, persons with BED are more vulnerable to depression (Hudson et al., 1988; Kuehnel \& Wadden, 1994; Marcus, Wing, \& Hopkins, 1988; Mussell et al., 1996; Spitzer et al., 1992; Telch \& Agras, 1994; Telch \& Stice, 1998; Wadden, Foster, \& Letizia, 1994; Yanovski, Nelson, Dubbert, \& Spitzer, 1993) and have a lower self-esteem (De Zwaan et al., 1994; Telch \& Agras, 1994; Telch \& Stice, 1998) than obese non-binge eaters.

For the most part, behavioral programs have not focused on psychological problems among obese participants. Critics of behavioral therapy focusing on 64 dieting for obese persons assert that the impact is typically devastating, sabotaging self-esteem and other psychological sequelae (Wooley \& Garner, 1991). Moreover, obese binge eaters perform less well in behavioral programs than do obese non-binge eaters. Obese binge eaters lose significantly less weight or rapidly regain it (Keefe, Wyshogrod, Weinberger, \& Agras, 1984; Marcus et al., 1988; Yanovski, Gormally, Leser, Gwirtsman, \& Yanovski, 1994) and do more frequently drop out of treatment (Keefe et al., 1984; Marcus et al., 1988; Yanovski et al., 1994). However, other studies found no significant differences between these two groups in weight loss or attrition in traditional behavioral therapy programs (Gladis et al., 1998; Porzelius, Houston, Smith, Arfken, \& Fisher, 1995).

Cognitive-behavioral therapy (CBT) is a broader form of therapy and consists of two major components: behavioral techniques and cognitive restructuring. In the behavioral part, normalizing of the eating pattern is established and persons are encouraged to moderate overall intake. In the cognitive part, maladaptive thoughts about dieting, shape, weight and more generic concerns about one's 
self-worth are challenged. At short-term there is evidence that obese binge eaters respond well to CBT analogous to the results from CBT among bulimia nervosa patients, at least as far as their binge eating is concerned, but there is no effect for weight (Agras et al., 1994; Marcus, Wing, \& Fairburn, 1995; Marcus et al., 1988; Porzelius et al., 1995; Smith, Marcus, \& Kaye, 1992; Telch, Agras, Rossiter, Wilfley, \& Kenardy, 1990; Wilfley et al., 1993). Until now, one study has included more psychological outcome measures like shape, weight, and eating concerns as outcome measures and found significant decreases on all concerns from pre- to posttreatment (Smith et al., 1992). Furthermore, the effects of a pure cognitive therapy had never been evaluated.

The purpose of this study was to compare the efficacy of a cognitive group intervention with a group behavioral intervention for obese binge eaters and obese non-binge eaters. The outcome measures of interest were: concerns about shape, weight, and eating, restraint, weight, binge eating, self-esteem, and depression. We hypothesized that obese women, and especially obese binge eaters in the cognitive condition would show greater improvements on all outcome measures, with an exception for weight, than in the behavior condition.

\section{METHOD}

\section{Participants}

Selected participants were 74 obese women. They were recruited from a group of respondents answering local newspaper advertisements that offered two university-based treatments for eating problems. In order to be included in the study female participants had to be between 18 and 50 years of age, with a Body Mass Index (BMI) of 27 or higher. Exclusion criteria included participation in a weight-loss program at the time of selection, current physical dependence on alcohol or drugs, psychosis, or pregnancy.

Obese women who responded to the advertisements were screened in a telephone interview for the inclusion and exclusion criteria. Interested and potentially eligible individuals $(\mathrm{N}=451)$ received the Questionnaire on Eating and Weight Patterns-revised (QEWP-r; Yanovski, 1993). This self-report questionnaire contains 28 items and contains also items that specifically test for the Diagnostic and Statistical Manual of Mental Disorders (DSM-IV; American Psychiatric Association, 1994) criteria for binge eating disorder (BED). Furthermore, questions on inclusion and exclusion criteria were added. After completion at home, the questionnaires were returned to the university $(\mathrm{N}=265)$.

If respondents met the BED criteria or did not have any binge episodes at all, they $(\mathrm{N}=145)$ were invited for a structured diagnostic interview that lasted about 1 hour and was conducted by a psychologist experienced in the assess- 
ment of eating disorders and obesity. The interviewer clarified and checked the DSM-IV items, especially those regarding quantity of food and extent of loss of control and the exclusion criteria were checked again. After the diagnostic interview, participants were weighed in street clothes, without shoes, on a balancebeam scale and their height was measured. The purpose of the study was explained, and written informed consent for the treatment study was obtained.

Respondents were categorized as non-binge eaters if they fulfilled none of the DSM-IV criteria for BED. The BED diagnosis was made if respondents met strict DSM-IV criteria for the disorder. We anticipated that comparisons of two extreme groups would most likely reveal relevant differences. Therefore, the major reason for exclusion was if respondents met some of the DSM-IV criteria, but not all. Other individuals canceled the interview appointment or did not appear at the interview, or decided not to participate in the program, primarily because of practical reasons. Finally, 37 women who met the proposed DSM-IV criteria for BED and 37 non-binge eaters comprised the definitive sample.

Participants were between 21 and 49 years $(M=38.3, S D=7.1)$. Their mean $\mathrm{BMI}$ was $33.1(\mathrm{SD}=4.3)$ and their mean weight was $93.5(\mathrm{SD}=13.5)$. Fifty-nine participants $(80 \%)$ were medically obese (BMI>30). Participants reported a mean duration of obesity of 21.1 years $(S D=8.5$; range 6 to 37 ). Binge eating disorder patients reported binge eating on an average of 4 days per week $(\mathrm{SD}=1.7)$. The mean duration of their binge eating was 12.5 years (SD $=6.4$; range 3 to 27 ).

\section{Study Design}

An experimental design was chosen consisting of a pre- and a postmeasurement and a follow-up measurement 6-months after treatment. Participants were recruited in two equal phases and were randomly assigned to one of the two 66 treatment conditions, taking binge status into account. Thus, this procedure resulted in four distinct groups; non-binge eaters who received behavioral therapy, binge eaters who received behavioral therapy, non-binge eaters who received cognitive therapy, and binge eaters who received cognitive therapy.

Selected participants were asked to complete pretest questionnaires at home and were invited for a semi-structured face-to-face interview 1-week before entering treatment. This interview lasted between 30 and 45 minutes. The purpose of the interview was to detect three dysfunctional thoughts regarding eating, weight, and shape, to check that all items of the questionnaires were filled out, to obtain weight, and that frequency of overeating was measured with the help of a 28-day calendar method (Fairburn \& Cooper, 1993). At postmeasurement and follow-up measurement, participants were asked again to complete the questionnaires at home and were invited for an interview. 


\section{Treatment}

\section{General aspects}

Both treatments were given in groups and all groups met for 15 weekly sessions of 150 minutes each. Ten groups, each consisting of 7 or 8 participants were assisted by one therapist. Eight therapists, experienced in the behavioral and cognitive therapy of eating disorders and/or obesity, participated in this study, each facilitating the treatment of at least one group. For both conditions, detailed treatment protocols were developed. All therapists received an intensive training in the protocol. Weekly consultation sessions of approximately one hour with each therapist were held to ensure therapists' compliance with treatment protocols. Treatment compliance of participants was checked by the therapists. Absence or presence of participants was recorded every session and completion of homework assignments was checked and they were gone over.

The two treatments shared the following features: Both were semi-structured and problem oriented, provided a coherent treatment rationale, were self-monitoring, and set homework assignments.

\section{Cognitive therapy (CT)}

The aim of this treatment was to change dysfunctional cognitions regarding shape, weight, eating, dieting and underlying self-schemas with help of the cognitive techniques outlined by Beck (1976). In the first session, the therapists explained the rationale of cognitive therapy. A binge/overeating circle was described as beginning with dysfunctional cognitions about shape, weight, eating, dieting or negative self-schemas.

Participants were instructed to self-monitor situations that trigger binge eating or overeating, and participants had to describe their thoughts. Examination of this monitoring was an important focus of therapy. During the first half of the therapy session, homework assignments of participants were discussed. In the second half, participants practiced identifying and challenging dysfunctional cognitions and setting up behavioral experiments.

The first stage of the therapy (six sessions) focused on identifying and altering dysfunctional cognitions regarding shape, weight and eating. The Socratic dialogue was used by the therapist to initialize active learning and to encourage a questioning, investigative attitude in the participants. Furthermore, participants were encouraged to engage in behavioral experiments designed to challenge their dysfunctional cognitions.

In the second stage (six sessions), cognitive tech-niques directed at identifying and challenging negative self-schemas which perpetuate disordered eating were emphasized. Negative self-schemas (i.e., 'If I go to the swimming pool, people will reject me' or 'If I gain weight, I am good for nothing') were identified and challenged and behavioral experiments were used.

The final stage (three sessions) largely concerned the maintenance of progress following the end of treatment. Marlatt \& Gordon (1985) have proposed that the Abstinence Violation Effect (AVE) accounts for some of the variability regarding 
relapse. The AVE model predicts that internal, stable and global attributions for a lapse increase the probability of overeating or binge eating. Participants were informed that lapses were normal and to be expected. The purpose of the cognitive restructuring procedure in the last phase was to reframe maladaptive attributions of causality regarding lapses to make it easier to regain control.

\section{Behavioral therapy (BT)}

The aim of the behavioral therapy was to learn a healthy eating pattern by having three meals each day and three planned snacks, decreasing fat intake, eating between 1500 to $1800 \mathrm{kcal}$ a day, increasing exercise habits, and recognizing and anticipating high risk situations.

In the first session, the therapists explained the rationale of behavioral therapy: To regain control over eating it is important to learn a healthy eating pattern, without excessive restrictive dieting. Participants were told that treatment needed first to eliminate binge eating and overeating patterns by establishing regular, healthy eating patterns and that weight control needed to be a secondary concern.

Participants were told to self-monitor their food intake and eating patterns, binge episodes, or episodes of overeating and the circumstances under which they occurred, such as time, presence of other people, and mood. Examination of the self-monitoring formed an important focus of therapy. In the first half of the session, homework assignments were discussed, and in the second half, nutritional information was given. Furthermore, participants were advised to make gradual changes in eating habits and exercise level. Therefore, weekly goals were set to change their eating habits, exercise level and general activity levels. The basic behavioral strategies were self-monitoring, goal-setting, and stimulus control techniques. Three forms of stimulus control techniques were used. First, participants learned stimulus control techniques whereby stimuli which resulted in eating were avoided (e.g., not going to a shop when hungry). Second, they learned techniques to anticipate high risk situations and to outline strategies to prevent eating, such as walking, talking with friends, or taking a shower. Third, participants learned self-reinforcement techniques, such as going to the movies or buying a magazine, and were positively reinforced by the therapist if they were not overeating. No attention was paid to dysfunctional cognitions. The distinctive features of cognitive therapy and behavioral therapy are summarized in Table 5.1.

\section{Measures}

Expectations of treatment were measured after session 1 with three statements on 0-100-mm Visual Analogue Scales (VASs) ranging from 0, ('totally disbelieved in'), to 100 , ('totally believed in'). The statements measured whether participants believed in the treatment rationale, whether they thought treatment would successfully eliminate their eating problem, and whether they could confidently recommend the treatment to their best friends.

The manipulation check consisted of a cognitive and a behavioral checklist, given 
Features of group cognitive therapy

Cognitive formulation of obesity

Modification of dysfunctional thoughts

Self-monitoring to identify cognitions concerning shape, weight, eating and the self

Cognitive restructuring and behavioral experiments to alter dysfunctional thoughts
Features of group behavior therapy
Behavioral formulation of obesity

Modification of a pattern of regular and healthy eating and exercising

Self-monitoring to identify eating pattern and high risk situations in which overeating could occur

Stimulus control procedures for healthy and regular eating and for anticipating high risk situations

to all participants at session 1 , session 8 , and session 15 . The cognitive checklist contained each participant's three dysfunctional cognitions concerning shape, weight, and eating that were identified in the semi-structured face-to-face interview before treatment. The cognitive checklist also contained seven general cognitions concerning shape, weight and eating. These seven cognitions were based on the most frequently mentioned cognitions earlier collected in our samples from obese populations seeking treatment for eating problems. Participants had to rate the degree of belief in these cognitions on $0-100 \mathrm{~mm}$ VASs. The VAS rating has face validity. The eating checklist measured regularity of eating (having breakfast, having lunch, having dinner) during the previous seven days. The number of meals was divided by seven to get the mean number of meals a day during the last week before Session 1, 8 and 15 .

Eating pathology was measured with the Eating Disorder ExaminationQuestionnaire (EDE-Q; Fairburn \& Beglin, 1994). The EDE-Q is a 36-item selfreport questionnaire version of the Eating Disorder Examination investigator-based interview, the so called Eating Disorder Examination (EDE; Fairburn \& Cooper, 1993). The EDE-Q is designed to assess the specific core psychopathology of eating disordered individuals and focuses on the past 28 days. We used four subscales of the EDE-Q: Shape Concern, Weight Concern, Eating Concern, and Restraint. Wilfley, Schwartz, Spurrell, and Fairburn (1997) found modest to good agreement between the self-report version and the investigator-based interview on these four subscales. However, they found low agreement when assessing binge eating. It was suggested that the performance of the EDE-Q would improve by clarifying the definitions of complex features, such as binge eating, with the help of an additional interview. Therefore, binge eating was assessed with the help of the interview method. Since Rossiter, Agras, Telch, \& Bruce (1992) proposed that recollecting the days with binge eating episodes is performed more accurately than recollecting the number of binge eating episodes, we measured the number 
of days in which an objective binge episode had occurred during the last 28-days.

Weight. Participants were weighed in street clothes, without shoes, on a balance beam scale.

Depression was measured with the Beck Depression Inventory (BDI; Beck, Ward, Mendelson, Mock, \& Erbaugh, 1961). The BDI is a 21-item self-report measure of severity of depressive symptomatology. The items are rated on a four-point scale ranging from 0 ('not depressed') to 3 ('severely depressed'), resulting in a range from 0 to 63 . Higher scores indicate more severe depression.

Self-esteem was measured with the Rosenberg's Self-Esteem Scale (RSE; Rosenberg, 1965), a 10-item questionnaire measuring global self-esteem, with special reference to the self-evaluation of the individual regarding an attitude of approval or disapproval. Each item is scored on a four-point scale ranging from strongly agree to strongly disagree, resulting in a range from 0 to 40 . A lower score indicates more positive self-esteem.

\section{Statistical procedure}

The analyses regarding treatment effects were by intention-to-treat. An attempt was made to obtain posttreatment and follow-up data on those who dropped out, but if this was not possible, their last available data were used instead. Ttests were used in order to reveal significant differences between obese binge eaters and obese non-binge eaters at pretreatment on demographic variables and outcome variables. The dependent variables were divided in eating pathology (dietary restraint and concerns about shape, weight, and eating), binge eating, weight, and generic psychopathology (depression and self-esteem). To investigate the effectiveness of both treatments the results were analyzed with 2 (Treatment: BT versus CT) $\times 2$ (Binge Status: obese binge eaters versus obese non-binge eaters) $\times 3$ (Time: pretreatment versus posttreatment versus follow70 up) repeated measures multivariate and univariate analysis of variance. If significant effects were found pairwise contrasts were conducted comparing pretreatment and posttreatment and between pretreatment and follow-up.

\section{RESULTS}

\section{Pretreatment}

To test whether there were pretreatment differences on demographic or outcome measures between obese binge eaters and obese non-binge eaters, t-tests were conducted. Obese binge eaters and obese non-binge eaters did not differ in Body Mass Index, weight or duration of obesity. However, obese binge 
eaters were significantly younger than obese non-binge eaters, $t(72)=5.90$, $\mathrm{p}<.001$. Furthermore, obese binge eaters and obese non-binge eaters differed significantly on all outcome measures $(\mathrm{p}<.001)$, except on restraint. Obese binge eaters were far more concerned about shape, weight, and eating, were more depressed and showed lower self-esteem than obese non-binge eaters.

\section{Treatment dropouts}

Ten participants (13.5\%) of a total of 74 participants dropped out of treatment: 3 binge eaters and 2 non-binge eaters from the CT group and 3 binge eaters and 2 non-binge eaters from the BT group. Reasons that participants gave for dropping out were: not losing enough weight $(\mathrm{N}=4)$, traveling time $(\mathrm{N}=2)$, and disrupting life-events $(\mathrm{N}=4)$. Dropout rates did not differ between cognitive and behavioral therapy, or between binge eaters and non-binge eaters. T-tests were conducted on demographic variables and outcome measures. Dropouts did not differ from those who completed treatment with regard to age, Body Mass Index, duration of obesity, duration of binge eating, eating and weight related dependent variables and depression. However, obese binge eaters who dropped out of treatment had a significantly lower self-esteem than obese binge eaters who didn't drop out of treatment $t(35)=3.74, p<.001$. Six of the ten treatment dropouts provided posttreatment data, five of the ten treatment dropouts provided followup data. All treatment completers provided posttreatment and follow-up data.

\section{Expectations of treatment and treatment compliance}

Credibility of the treatment rationale was high in both treatments (CT $=85.7$ and $\mathrm{BT}=83.4)$. The rating on confidence that treatment would be successful was 84.7 in the CT group and 79.8 in the BT group. Recommending treatment to a friend scored 83.9 in the CT group and 80.2 in the BT group. T-tests revealed no significant differences between the cognitive therapy and the behavioral therapy on any of these questions. These results suggest that the treatments were presented to participants in an equally credible fashion by the therapists and that both groups held high and equal expectations for improvement.

Treatment compliance was high. Participants in the behavioral therapy attended 13.3 treatment sessions $(89 \%)$ and participants in the cognitive therapy attended 13.8 sessions (92\%). A t-test revealed no significant differences in attendance between treatments.

\section{Manipulation check}

Table 5.2 shows means and standard deviations of the different manipulation checks at session 1, 8 and 15. A 2 (Treatment: BT versus CT) by 3 (Time: ses- 
Table 5.2 Means and standard deviations at session I, session 8, and session 15, for the manipulation check

Manipulation check

Cognitive therapy

Behavioral therapy

\begin{tabular}{|c|c|c|c|c|c|}
\hline Session I & Session 8 & Session 15 & Session I & Session 8 & Session 15 \\
\hline ea & Mean (SD) & Mean (SD) & Mean (SD) & Mean (SD) & Mean (SI \\
\hline
\end{tabular}

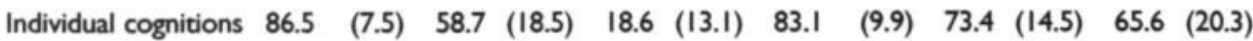

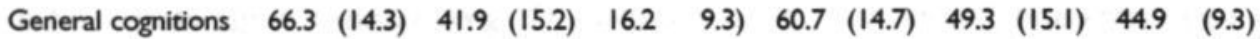

$\begin{array}{lllllllllllll}\text { Regular eating' } & 2.7 & (0.4) & 2.7 & (0.4) & 2.7 & (0.4) & 2.5 & (0.7) & 2.9 & (0.2) & 3.0 & (0.1)\end{array}$

I Mean number of meals per day during one week

sion 1 versus session 8 versus session 15) repeated measures ANOVA conducted on the cognition checklist revealed a significant Time effect on the individual cognitions, $\mathrm{F}(2,124)=235.59, \mathrm{p}<.001$, and the general cognitions, $\mathrm{F}(2,124)=$ $184.43, \mathrm{p}<.001$, indicating that belief in dysfunctional cognitions decreased over time. Both treatments had effects on dysfunctional cognitions. The change in the behavioral therapy might have occurred because the change in behavior might indirectly have influenced cognitions. However, a Time $\mathrm{x}$ Treatment interaction effect was found on the individual cognitions, $F(2,124)=84.16, p<.001$, and the general cognitions, $\mathrm{F}(2,124)=50.67, \mathrm{p}<.001$, showing a greater decrease in dysfunctional cognitions over time in the cognitive therapy. Further repeated pairwise contrast analyses were conducted to test whether the means were signi72 ficant different within treatment over time. For individual cognitions significant Time $\mathrm{x}$ Treatment interaction effects were found between session 1 and session $8, \mathrm{~F}(1,62)=24.00, \mathrm{p}<.001$, and between session 1 and session $15, \mathrm{~F}(1,62)=$ $150.40, \mathrm{p}<.001$. For general cognitions significant Time $\mathrm{x}$ Treatment interaction effects were found between session 1 and session $8, F(1,62)=12.25, p<.001$, and between session 1 and session $15, \mathrm{~F}(1,62)=86.06, \mathrm{p}<.001$.

A 2 (Treatment) by 3 (Time) repeated measures ANOVA on the eating habits checklist revealed a significant Time effect for regular eating, $F(2,124)=13.68$, $\mathrm{p}<.001$, which was determined by a significant Time $\mathrm{x}$ Treatment interaction, $\mathrm{F}(2,124)=12.13, \mathrm{p}<.001$, indicating that participants in the behavioral therapy acquired a more regular eating pattern over time, while participants in the cognitive therapy remained constant. Further repeated pairwise contrast analyses were conducted to test whether the means were significantly different within treatment over time. For eating habits significant Time $\mathrm{x}$ Treatment interaction effects were found between session 1 and session $8, F(1,62)=11.17, p<.01$ and between session 1 and session $15, F(1,62)=14.86, p<.001$. 


\section{Dietary restraint and concerns about shape, weight, and eating}

Table 5.3 shows the means and standard deviations for all outcome measures at pretreatment, posttreatment, and at 6-month follow-up.

The repeated measures MANOVA performed on the pretreatment, posttreatment and follow-up scores on the four subscales of the EDE-Q showed a highly significant multivariate main effect for Time, $F(8,280)=17.03, p<.001$. Univariate contrast analyses comparing pretreatment with posttreatment scores were further conducted to reveal differences on each subscale of the EDE-Q. Three of the four subscales of the EDE-Q showed significant Time effects $(\mathrm{p}<.001)$, except for restraint. Comparisons between pretreatment and followup scores yielded similar results. Concerns about shape, weight, and eating decreased over time.

Furthermore, there was a significant multivariate interaction effect for Treatment $\mathrm{x}$ Time, $\mathrm{F}(8,280)=2.76, \mathrm{p}<.01$. Additional univariate analyses revealed Treatment $\mathrm{x}$ Time interactions between pretreatment and posttreatment for shape concern; $F(1,71)=19.84, \mathrm{p}<.001$; weight concern, $F(1,71)=$ 17.22, $\mathrm{p}<.001$, eating concern, $\mathrm{F}(1,71)=9.18, \mathrm{p}<.01$; and restraint, $\mathrm{F}(1,71)$ $=3.51, \mathrm{p}=.07$. Inspection of the means revealed that the cognitive therapy was superior to the behavioral therapy for all subscales. Univariate contrast analyses comparing pretreatment and follow-up revealed significant Treatment $\mathrm{x}$ Time interactions for shape concern, $F(1,71)=4.47, \mathrm{p}<.05$; weight concern, $\mathrm{F}(1,71)=10.34, \mathrm{p}<.001$, and eating concern, $\mathrm{F}(1,71)=5.61, \mathrm{p}<.05$. Inspection of the means revealed that the cognitive therapy was superior to the behavioral therapy for all three subscales. No significant Treatment $\mathrm{x}$ Time interaction effect was found for restraint.

Finally, there was a highly multivariate interaction effect for Binge Status $\mathrm{x}$ Time, $F(8,280)=6.60, p<.001$. Univariate contrast analyses between pretreatment and posttreatment showed significant Binge Status $\mathrm{x}$ Time effects for shape concern, $\mathrm{F}(1,71)=15.02, \mathrm{p}<.001$; weight concern, $\mathrm{F}(1,71)=4.98$, $\mathrm{p}<.05$; and eating concern, $\mathrm{F}(1,71)=41.72, \mathrm{p}<.001$. Obese binge eaters improved more than obese non-binge eaters between pretreatment and posttreatment. No significant Binge Status x Time interaction effect was found for restraint. Univariate contrast analyses between pretreatment and follow-up showed significant Binge Status $\mathrm{x}$ Time effects for shape concern, $\mathrm{F}(1,71)=$ $11.34, \mathrm{p}<.01$; weight concern, $\mathrm{F}(1,71)=3.24, \mathrm{p}=.08$; and eating concern, $F(1,71)=33.30, p<.001$. Obese binge eaters improved more than obese nonbinge eaters between pretreatment and follow-up. Again, no significant Binge Status $\mathrm{x}$ Time interaction effect was found for restraint. Figure 5.1 shows the mean scores for obese bingers and obese non-bingers on all EDE subscales before and after CT and BT intervention and at follow-up. 
Table 5.3 Means and standard deviations at pretreatment, posttreatment and Follow-Up for all outcome measures

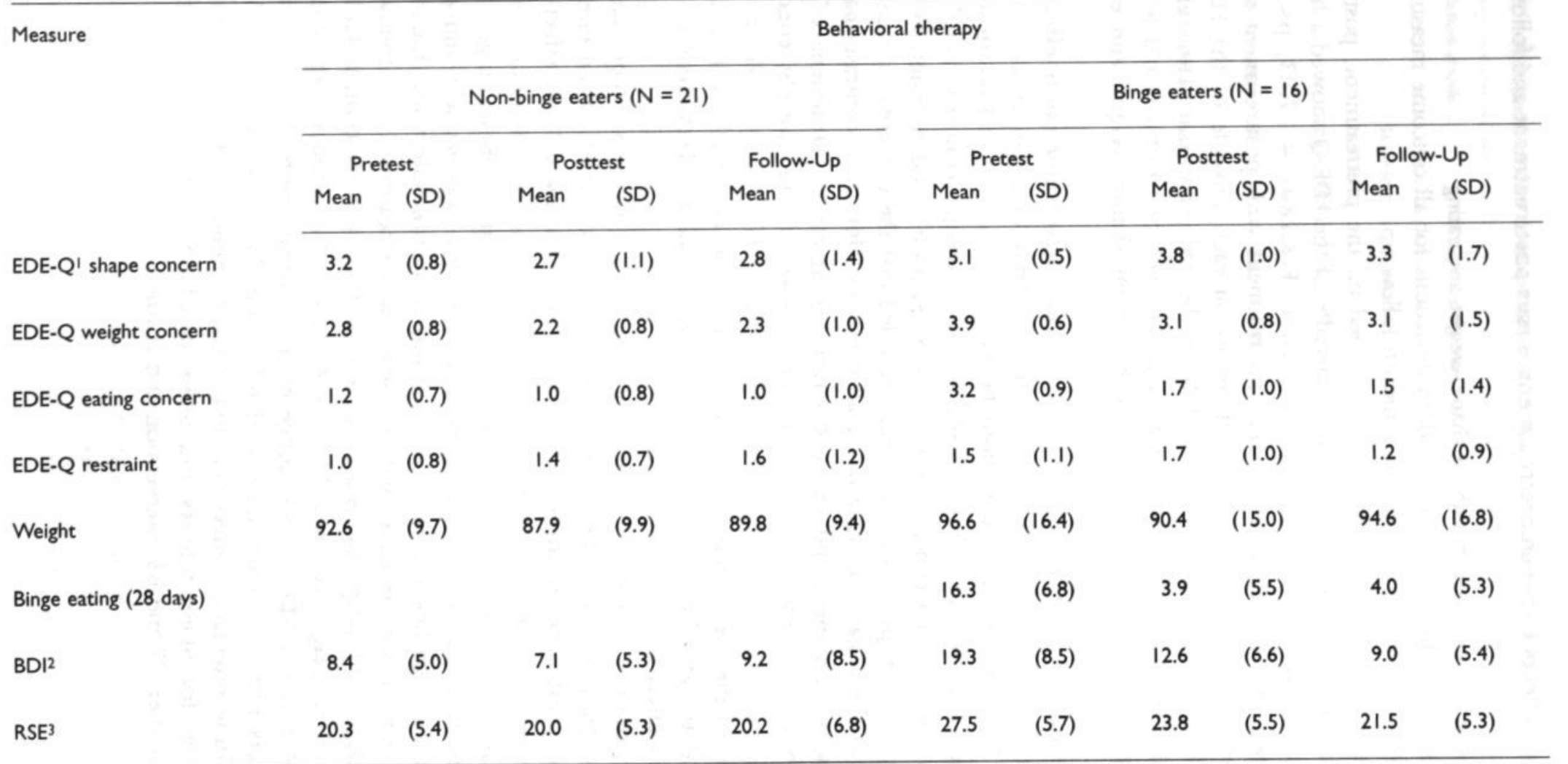

1 EDE-Q = Eating Disorder Examination-Questionnaire; ${ }^{2}$ BDI= Beck Depression Inventory. ${ }^{3}$ RSE $=$ Rosenberg Self-Esteem Scale (table continues) 
Table 5.3 Means and standard deviations at pretreatment, posttreatment and Follow-Up for all outcome measures

\begin{tabular}{|c|c|c|c|c|c|c|c|c|c|c|c|c|}
\hline \multirow{4}{*}{ Measure } & \multicolumn{12}{|c|}{ Cognitive therapy } \\
\hline & \multicolumn{6}{|c|}{ Non-binge eaters $(N=16)$} & \multicolumn{6}{|c|}{ Binge eaters $(N=21)$} \\
\hline & \multicolumn{2}{|c|}{ Pretest } & \multicolumn{2}{|c|}{ Posttest } & \multicolumn{2}{|c|}{ Follow-Up } & \multicolumn{2}{|c|}{ Pretest } & \multicolumn{2}{|c|}{ Posttest } & \multicolumn{2}{|c|}{ Follow $U_{p}$} \\
\hline & Mean & (SD) & Mean & $(S D)$ & Mean & (SD) & Mean & $(\mathrm{SD})$ & Mean & (SD) & Mean & (SD) \\
\hline EDE-QI shape concern & 3.3 & $(1.1)$ & 1.9 & $(1.1)$ & 1.9 & $(1.2)$ & 5.1 & $(0.6)$ & 2.8 & $(1.3)$ & 2.9 & $(1.7)$ \\
\hline EDE-Q weight concern & 2.7 & $(0.9)$ & 1.5 & $(1.0)$ & 1.5 & $(1.0)$ & 4.2 & $(0.6)$ & 2.2 & $(1.1)$ & 2.3 & $(1.5)$ \\
\hline EDE-Q eating concern & 1.4 & $(0.7)$ & 0.7 & $(1.0)$ & 0.6 & $(0.6)$ & 3.6 & $(1.2)$ & 1.2 & $(0.7)$ & 1.2 & $(1.2)$ \\
\hline$E D E-Q$ restraint & 1.1 & $(0.9)$ & 1.1 & $(1.2)$ & 0.9 & $(1.0)$ & 1.5 & (I.I) & 1.2 & $(0.9)$ & 1.5 & (1.4) \\
\hline Weight & 88.8 & (11.1) & 88.3 & $(12.3)$ & 89.1 & $(13.1)$ & 95.5 & (15.5) & 94.2 & $(15.5)$ & 95.4 & $(16.7)$ \\
\hline Binge eating ( 28 days) & & & & & & & 15.8 & $(6.7)$ & 1.6 & $(3.8)$ & 1.5 & (4.3) \\
\hline$\left.\mathrm{BD}\right|^{2}$ & 7.3 & $(5.1)$ & 4.3 & $(4.5)$ & 5.6 & $(5.4)$ & 19.2 & $(6.5)$ & 10.0 & $(9.1)$ & 10.9 & $(11,0)$ \\
\hline RSE $^{3}$ & 20.9 & $(4.7)$ & 17.4 & (5.5) & 17.4 & $(5.4)$ & 27.8 & $(4.2)$ & 21.4 & $(5.2)$ & 22.7 & $(7.2)$ \\
\hline
\end{tabular}

1 EDE-Q $=$ Eating Disorder Examination-Questionnaire; ${ }^{2}$ BDI= Beck Depression Inventory; ${ }^{3}$ RSE $=$ Rosenberg Seff-Esteem Scale 

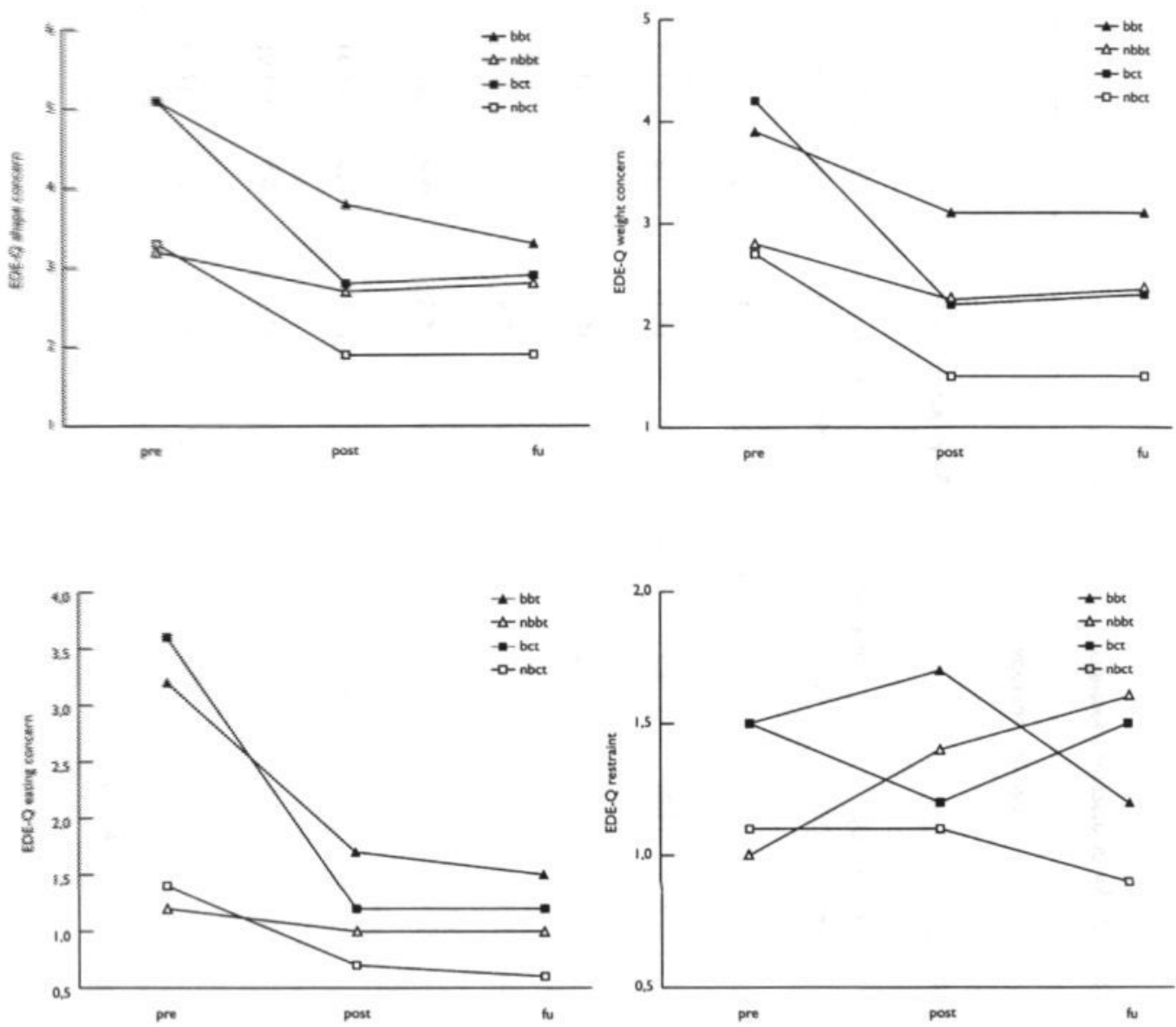

Figure 5.I Mean ratings before and ofter $C T$ and BT and at follow-up for obese binge eaters (B) and obese nonbinge eaters (NB) on concerns about shape (5.Ia), weight (5.I b), eating (5.Ic) and restraint (5.I d).

\section{Weight}

Results of the 2 (Treatment) $\times 2$ (Binge Status) $\times 3$ (Time) repeated measures ANOVA performed on the pretreatment, posttreatment and follow-up scores for weight yielded a significant effect for Time, $\mathrm{F}(2,142)=20.53, \mathrm{p}<.001$. Univariate contrast analyses yielded significant Time effects between pretreatment and posttreatment, $\mathrm{F}(1,71)=90.22, \mathrm{p}<.001$, and between pretreatment and follow-up, $\mathrm{F}(1,71)=4.55, \mathrm{p}<.05$.

Furthermore, there was an interaction effect for Treatment $x$ Time, $F(2,142)$ $=10.52,<.001$. Univariate contrast analyses showed significant Treatment $\mathrm{x}$ Time interaction effects between pretreatment and posttreatment, $F(1,71)=$ 47.06, p<.001. Participants in the behavioral therapy lost $5.5 \mathrm{~kg}$, while participants in the cognitive therapy lost $0.8 \mathrm{~kg}$. Univariate contrast analyses showed 


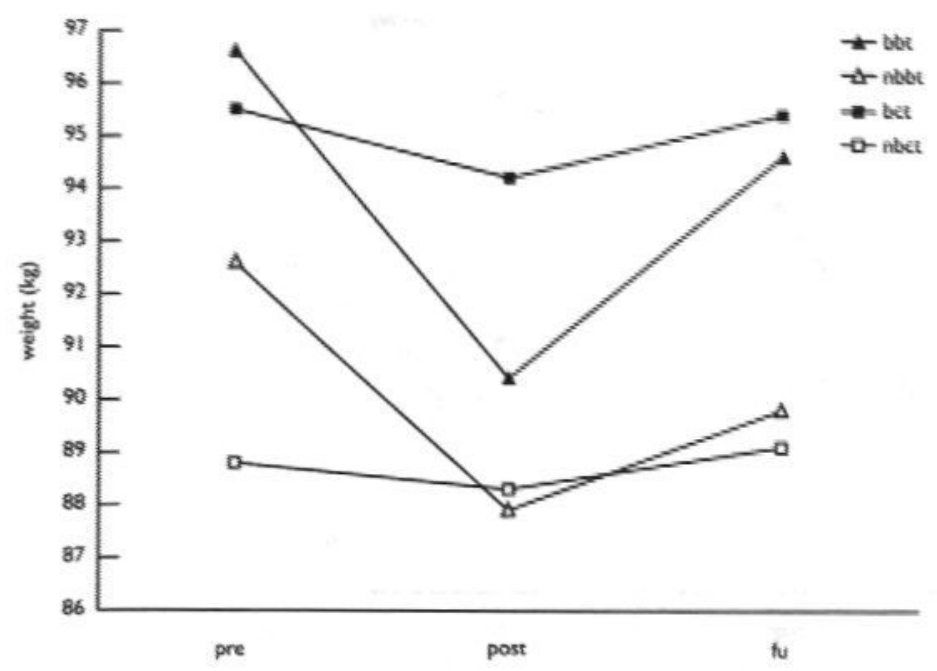

Figure 5.2 Mean ratings before and after $C T$ and $B T$ and at follow-up for obese binge eaters $(B)$ ond obese non-binge eaters (NB) on weight.

also significant Treatment $\mathrm{x}$ Time interaction effects between pretreatment and follow-up, $F(1,71)=4.87, p<.05$. Participants in the behavioral therapy lost $2.4 \mathrm{~kg}$, while participants in the cognitive therapy gained $0.1 \mathrm{~kg}$. Finally, there was no significant multivariate interaction effect for Binge Status $\mathrm{x}$ Time between pretreatment, posttreatment and follow-up. However, additional t-tests showed that obese binge eaters showed a significant weight gain from posttreatment to follow-up, $\mathrm{t}(36)=2.82, \mathrm{p}<.01$, while obese non-binge eaters showed no significant weight gain from posttreatment to follow-up. Figure 5.2 shows the mean ratings on weight before and after CT and BT intervention and at follow-up for obese binge eaters and obese non-binge eaters.

\section{Binge eating}

Results of the 2 (Treatment) x 3 (Time) repeated measures ANOVA performed on the pretreatment, posttreatment and follow-up scores for objective binge episodes yielded a significant effect for Time, $\mathrm{F}(2,70)=82.78, \mathrm{p}<.001$. Univariate contrast analyses yielded highly significant Time effects between pretreatment and posttreatment, $\mathrm{F}(1,35)=89.36, \mathrm{p}<.001$, and between pretreatment and follow-up, $\mathrm{F}(1,35)=101.95, \mathrm{p}<.001$.

Results of the 2 (Treatment) x 3 (Time) repeated measures ANOVA performed on the pretreatment, posttreatment and follow-up scores for objective binge episodes yielded no significant effect for Treatment $\mathrm{x}$ Time. At posttreatment participants from the cognitive therapy had reduced bingeing by $90 \%$ and participants from the behavioral therapy had reduced bingeing by $76 \%$. Sixty- 


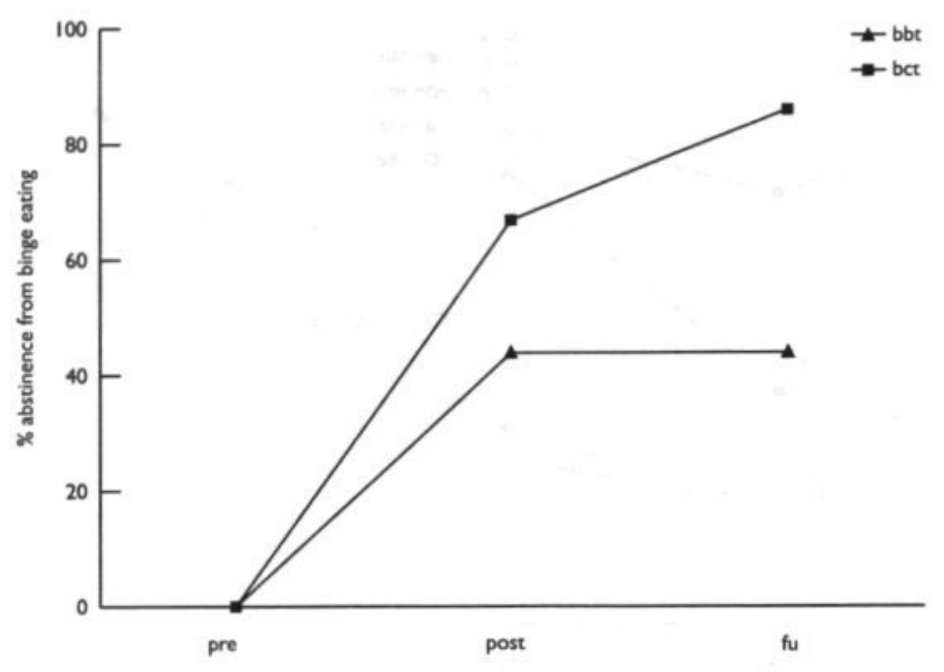

Figure 5.3 Mean ratings before and after $C T$ and $B T$ and at follow-up for obese binge eaters (B) on abstinence from binge eating.

seven percent of obese binge eaters abstained from binge eating after cognitive therapy, compared to $44 \%$ abstinence after behavioral therapy. At the end of treatment four obese binge eaters in the behavioral therapy still fulfilled all DSM-IV criteria of binge eating disorder, while two of the participants in the cognitive therapy still fulfilled all criteria. At follow-up participants in the cognitive therapy had reduced bingeing by $91 \%$ and participants in the behavioral therapy had reduced bingeing by $75 \%$. A greater percentage of the participants in the cognitive condition were abstinent from binge eating (86\%), as compared to participants who received behavioral therapy (44\%). A Fisher exact test revealed that the proportion of participants abstaining from binge eating at follow-up in the cognitive therapy was significantly higher than in the behavioral group $(p<.01)$. At follow-up four obese binge eaters from the behavioral therapy and two obese binge eaters from the cognitive therapy still fulfilled all DSM-IV criteria of binge eating disorder. Figure 5.3 shows the abstinence rates from binge eating before and after $\mathrm{CT}$ and $\mathrm{BT}$ intervention and at follow-up.

\section{Depression and self-esteem}

The repeated measures MANOVA performed on the pretreatment, posttreatment and follow-up scores of the BDI and RSE showed a significant multivariate main effect for Time, $\mathrm{F}(4,284)=14.49, \mathrm{p}<.001$. Univariate contrast analyses comparing pretreatment with posttreatment scores showed significant Time effects on depression, $\mathrm{F}(1,71)=70.40, \mathrm{p}<.001$ and self-esteem, $\mathrm{F}(1,71)=76.19$, $\mathrm{p}<.001$, while the comparison of pretreatment scores with follow-up scores 
yielded a similar result for depression, $\mathrm{F}(1,71)=31.39, \mathrm{p}<.001$ and selfesteem, $\mathrm{F}(1,71)=34.81, \mathrm{p}<.001$.

Furthermore, a marginally significant multivariate interaction effect for Treatment $\mathrm{x}$ Time was found, $\mathrm{F}(4,284)=2.15, \mathrm{p}=.08$. Univariate contrast analyses comparing pretreatment with posttreatment scores showed a significant Treatment $x$ Time effect for self-esteem, $F(1,71)=13.46, \mathrm{p}<.001$, and marginally for depression, $F(1,71)=2.89, \mathrm{p}<.10$. Inspection of the means showed that the cognitive therapy was superior to the behavioral therapy in enhancing self-esteem between pretreatment and posttreatment. The comparison of pretreatment scores with follow-up scores yielded non-significant Treatment $\mathrm{x}$ Time results for self-esteem, indicating that the initial superiority of the cognitive therapy was not present at follow-up.

Finally, there was a significant multivariate Binge Status x Time effect, $\mathrm{F}(4,284)=8.23, \mathrm{p}<.001$. Univariate contrast analyses comparing pretreatment with posttreatment scores revealed significant Binge Status $\mathrm{x}$ Time effects for self-esteem, $\mathrm{F}(1,71)=15.84, \mathrm{p}<.001$ and depression, $\mathrm{F}(1,71)=22.73$, $\mathrm{p}<.001$, indicating that obese binge eaters showed more improvement than obese non-binge eaters. This was also the case at follow-up: the comparison of pretreatment scores with follow-up scores yielded significant Binge Status $x$ Time results for self-esteem, $\mathrm{F}(1,71)=9.90, \mathrm{p}<.01$ and depression, $\mathrm{F}(1,71)=$ $26.84, \mathrm{p}<.001$.

\section{Per-protocol analyses}

At posttreatment $13.5 \%$ of the participants had dropped out of the study. Additional analyses were repeated on all dependent variables. There were essentially no differences between the per-protocol analyses and the intent-to-treat analyses discussed earlier.

\section{DISCUSSION}

The purpose of the present study was to investigate the effectiveness of a cognitive intervention compared to a behavioral intervention for obese women with and without binge-eating problems. The main hypothesis tested was that cognitive therapy would improve concerns about shape, weight, and eating, restraint, overeating, self-esteem and depression more than behavioral therapy. In both treatments, the scores on nearly all outcome measures were improved between pretreatment and posttreatment and pretreatment and follow-up, except for restraint. The cognitive and behavioral therapy were both very effective in reducing a broad spectrum of complaints. However, the cognitive therapy was 
more effective in reducing concerns about shape, weight, and eating concerns, as well as in reducing restraint and in improving self-esteem. At 6-month follow-up these results were maintained for shape, weight, and eating concerns. At posttreatment there were no differences in abstinence from binge eating or binge eating episodes between the behavioral and cognitive therapy. However, at 6-month follow-up, the proportion of participants abstaining from binge eating in the cognitive therapy was significantly higher than in the behavioral group. The behavioral therapy was more effective in reducing weight at posttreatment. However, at follow-up participants gained weight. Finally, the results showed that obese binge eaters benefited more from treatment than obese nonbinge eaters on measures of concerns about shape, weight, and eating, selfesteem, and depression. Obese binge eaters may have experienced more clinical change than obese non-binge eaters due to floor effects in the latter group. There were no differences between obese binge eaters and obese non-binge eaters on measures of restraint.

From the manipulation checks, it was discovered that the cognitive therapy and the behavioral therapy specifically targeted cognitions and eating behavior, respectively. Participants in the cognitive condition reduced their degree of belief in the individual and general dysfunctional cognitions to a greater extent than participants in the behavioral condition. The eating pattern became more regular for participants in the behavioral condition than for participants in the cognitive condition. In addition, the intervention techniques produced the greatest changes in the corresponding outcome measures. The participants judged both treatments as being equally appropriate for their eating problem and there were no differences in expectation of improvement across the treatments. Therefore, the results are likely to have arisen from differences in the techniques employed rather than from non-specific factors. These findings underline the statement that behavioral and cognitive therapy may effect improvement through different mechanisms, as has been suggested in studies of 80 bulimia nervosa and other disorders (Agras, Schneider, Arnow, Raeburn, \& Telch, 1989). In our study we used VAS scales to measure change in cognitions during treatment. In further research it would be better to use a psychometrically validated measure for measuring cognitions. Although the VAS ratings have only face validity, this list of individualized cognitions has proven to be useful in exploring the diverse content of cognitions in eating disordered and obese patients (Nauta, Hospers, Jansen, \& Kok, 2000).

In this study eating pathology was measured with the EDE-Q. According to Fairburn and colleagues (1993), a clinically significant change on the EDE can be operationalized as whether participants meet scores within one standard deviation of the scores obtained from persons who do not seek treatment. In a recent study, Telch and Stice (1998) report normative data on the EDE-Q for sixty nontreatment seeking obese persons. In our study, the mean scores obtained from all obese binge eaters and obese non-binge eaters of the cognitive therapy as well as the behavioral therapy met at posttreatment scores within one standard deviation of the scores obtained from obese persons who did not seek treatment. 
The binge abstinence rates, particularly in the cognitive intervention were high. After treatment, $67 \%$ of obese binge eaters abstained from binge eating in the cognitive therapy, compared to $44 \%$ abstinence in the behavioral therapy. At follow-up $86 \%$ of the participants in the cognitive condition were abstinent from binge eating and $44 \%$ of the participants in the behavioral therapy. Data from trials administering CBT suggest abstinence rates in obese binge eaters ranging from 28\% to 79\% (Agras et al., 1994; Agras et al., 1995; Marcus et al., 1995; Smith et al., 1992; Telch et al., 1990; Wilfley et al., 1993), with a mean abstinence rate of approximately $50 \%$. An explanation for the present high abstinence rates, particularly in the long-term for the pure cognitive therapy is that the cognitive component in CBT is at least briefer and may be less intensive than in a pure cognitive therapy. The learning and implementation of the cognitive strategies might require longer time intervals and extensive training. In that case, addition of more cognitive elements in the existing behavioral therapy programs shows promise as a means of enhancing maintenance.

Restraint is believed to be a critical antecedent of binge eating (e.g., Polivy \& Herman, 1995; Telch \& Agras, 1993). In this study, restraint was measured with the Restraint subscale of the EDE-Q. The EDE-Q assesses both intent to restrict calories and actual efforts to restrain food intake. It was, however, found that obese binge eaters had the same scores on restraint as obese non-binge eaters in this study. This result was also found in similar research in which the EDErestraint subscale was used (Wilson et al., 1993). After treatment the level of restraint was increased in the behavioral therapy, while binge eating was reduced. Our data support the idea of Castonguay, Eldredge and Agras (1995) that for obese binge eaters chaotic eating patterns may play a larger role in triggering binge eating than the intention to restrict calories and actual efforts to restrain food intake. Further investigation of the complex relationship between binge eating, chaotic eating patterns and restraint in obese binge eaters seems warranted.

Furthermore, for weight loss it seems warranted that the caloric intake must be reduced and therefore the level of restraint increased. The baseline levels of restraint, as measured with the EDE-Q, were low for participants in the behavioral and the cognitive therapy. The participants in the behavioral therapy increased the level of restraint more than participants in the cognitive therapy. Participants in the behavioral therapy lost an average of $5.5 \mathrm{~kg}$ directly after treatment. Presumably, this might explain the superiority of BT on weight loss directly after treatment. Unfortunately, the weight loss achieved in the behavioral therapy was not maintained. At six-month follow-up almost all participants in the behavioral therapy gained weight. The weight gain occurred earlier than in most other studies. However, almost all studies found that weight is gradually regained over time after 1-year follow-up (e.g., Garner \& Wooley, 1991; Wilson, 1994). It would be valuable to examine the optimum level for caloric intake for obese binge eaters and obese non-binge eaters in which there is no tendency to binge eat and in which weight loss is possible and could be maintained. Participants in the cognitive therapy hardly lost any weight. This is in accordance with other research that evaluated the effectiveness of CBT interven- 
tion for obese binge eaters and that also found no effects for weight after treatment (Agras et al., 1994; Marcus et al., 1995; Marcus et al., 1988; Porzelius et al., 1995; Smith et al., 1992; Telch et al., 1990; Wilfley et al., 1993). Finally, obese binge eaters seemed to regain more weight than obese non-binge eaters between posttreatment and follow-up. It has been hypothesized that obese binge eaters tend to overeat because they have lost their sensitivity to internal cues of hunger through bingeing (Howard \& Porzelius, 1999). It might be that obese binge eaters who regained weight were not sensitive to internal cues of hunger and satiety, and still had a propensity to overeat. Treatment components aimed at restoring the sensitivity might be very helpful, like the appetite awareness training (Craighead \& Allen, 1995).

In interpreting the results of this study, certain potential limitations of this should be considered. First, the generalizability of the results to all obese persons may be limited. The obese women who agreed to participate in this study were probably not a representative sample of the population of all obese women. Most were dissatisfied with dieting and wanted a different treatment for their eating problem than another dieting program. A second limitation of this study was the lack of a no treatment control group or a placebo control condition. The positive effects of this study may be due to a placebo effect, although six controlled studies showed that behavioral therapy is superior to a no treatment control group (Agras et al., 1995; Marcus et al., 1995, Eldredge et al., 1997; Peterson et al., 1998; Telch et al., 1990, Wilfley et al., 1993). Furthermore, it is very difficult to find a placebo control group that imitated the experimental treatment in all ways, except the specific active factor (Omer \& London, 1989).

It remains important to distinguish between controlled research studies on treatment effectiveness, and their use in clinical practice. Therefore the practical implications of this study need to be considered. The cognitive therapy was more effective, at least as far as dysfunctional cognitions towards shape, weight, 82 and eating, binge eating, and self-esteem were concerned. Inclusion of cognitive techniques could be considered in future treatment packages. Both treatments were not very effective in producing weight loss at 6-month follow-up. This result underlines the statement that only focusing on weight loss is yet an inappropriate goal for obese persons. For obese persons it is important to assess the full range of (eating) problems. Outcome measures may have to be broadened beyond simple weight loss in obese persons and binge frequency and weight loss in obese binge eaters, to include a broader assessment of the range of eating pathology. More empirical studies looking at a broad spectrum of outcome differences, including cognitive and psychological changes between different obese subgroups are needed. Different treatment techniques can be used to resolve the intriguing question of which technique is best for which obese subgroup to decrease overeating and body weight on the one hand and to improve cognitive and emotional functioning on the other hand. 


\section{ONE-YEAR FOLLOW-UP EFFECTS OF TWO OBESITY TREATMENTS ON PSYCHOLOGICAL WELL-BEING AND WEIGHT}

This section is a slightly adjusted version of the article:

Nauta, H., Hospers, H., \& Jansen, A. (200I). One-year follow-up effects of two obesity treatments on psychological well-being and weight. British Journal of Health Psychology, 6(3), 27I-284.

\section{INTRODUCTION}

Behavioral treatments for obese persons which focus on weight loss generally succeed in the short-term, but are often not very effective in maintaining weight loss in the long-term (e.g., Bennett, 1986; Garner \& Wooley, 1991; Wadden, 1993; Wilson, 1994). Despite diverse attempts to improve long-term maintenance, almost all participants return to their baseline weights (e.g., Perri et al., 1987; Perri, Nezu, Patti, \& McCann, 1989; Wilson, 1994). Moreover, several researchers have found that dieting itself has negative effects as well. Frequent dieting might be associated with increased cardiovascular and all-cause mortality (Blair, Shaten, Brownell, Collins, \& Lissner, 1993; Lissner et al., 1991), and results in pathological changes in cognition and affect (Brownell \& Rodin, 1994; Foreyt et al., 1995; Friedman \& Brownell, 1995; Polivy \& Herman, 1992). The literature is inconclusive as to the causal role that dieting plays in the development of binge eating in obese persons (Howard \& Porzelius, 1999). However, in a prospective study of Telch and Agras (1993), it was found that caloric restriction leads to binge eating episodes in obese persons. 
This discouraging outcome of obesity treatment and the negative effects of dieting has led to an acknowledgement that weight loss may be an inappropriate first goal for many obese persons. There is some evidence that it may be more prudent to focus directly on psychological well-being rather than dieting. Therefore, researchers and clinicians are beginning to call for non-dieting approaches that focus upon outcome goals other than weight loss (Ciliska, 1990; Garner \& Wooley, 1991; Polivy \& Herman, 1992, Wardle, 1995). The focus of a non-dieting approach will be on the improvement of psychological well-being, on restructuring dysfunctional cognitions regarding body weight and shape, and on restoring normal eating patterns, versus weight loss per se.

Obese individuals presenting for treatment of obesity display a higher prevalence of psychological problems than obese persons not seeking treatment (Friedman \& Brownell, 1995; Miller \& Downey, 1999). An important development in the study of obesity is the recognition that between $20 \%$ and $46 \%$ of obese patients seeking treatment report moderate to severe problems with binge eating (Bruce \& Wilfley, 1996; Gormally, Black, Daston, \& Rardin, 1982; Marcus, Wing, \& Lamparski, 1985; Spitzer et al., 1992; Telch, Agras, \& Rossiter, 1988). This subgroup of obese persons displays even more severe psychological problems than obese non-binge eaters. Obese binge eaters show more concerns about shape, weight, and eating than obese non-binge eaters (Eldredge \& Agras, 1996; Wilson, Nonas, \& Rosenblum, 1993), at levels that are comparable to those mentioned by bulimia nervosa patients (Marcus, Smith, Santelli, \& Kaye, 1992). Furthermore, persons with binge eating disorder are more vulnerable to depression (Hudson et al., 1988; Kuehnel \& Wadden, 1994; Marcus, Wing, Hopkins, 1988; Mussell et al., 1996; Spitzer et al., 1992; Telch \& Agras, 1994; Wadden, Foster, \& Letizia, 1994; Yanovski, Nelson, Dubbert, Spitzer, 1993) and have a lower self-esteem (De Zwaan et al., 1994; Telch \& Agras, 1994) than obese non-binge eaters.

Focussing on psychological well-being, healthy cognitions regarding body 84 weight and shape, and non-dieting might lead to more lasting improvements and might have positive effects on weight in the long run than dieting approaches, considering that caloric restriction is an important determinant of binge eating or overeating. A non-dieting approach will be advantageous over dieting approaches in the treatment of obesity and obese binge eaters in particular. Improvement of psychological well-being and healthy cognitions regarding body weight and shape might decrease overeating elicited by feeling bad and, in that way, facilitate weight loss.

Long-term follow-up results of non-dieting approaches for obese persons have rarely been reported. To date, only five studies of non-dieting treatments have reported follow-up results of 1-year or more. Of these, two focussed on a modified behavioral therapy with cognitive elements for obese binge eaters and obese non-binge eaters (Marcus et al., 1988; Porzelius, Houston, Smith, Arfken, \& Fisher, 1995) and three studies reported results of cognitive-behavioral therapy for obese binge eaters in particular (Agras, Telch, Arnow, Eldredge, \& Marnell, 1997; Wilfley et al., 1993; Goodrick, Poston, Kimball, Reeves, \& 
Foreyt, 1998). A central prediction of the cognitive model is that weight loss will not take place and binge eating will follow as concerns about shape, weight, and eating are maintained or increased (Fairburn, Marcus, \& Wilson, 1993). The primary outcome measures in the aforementioned studies were weight loss and in the case of obese binge eaters, frequency of binge eating. Interestingly, attitudes toward shape, weight, and eating were not reported. In three studies, objective binge episodes were measured and in all these studies a reduction in objective binge episodes was found, in the short and long-term (Agras et al., 1997; Porzelius et al., 1995; Wilfley et al., 1993). The results were less clear concerning weight loss. Weight loss tended to be regained at long-term follow-up (Goodrick et al., 1998; Marcus et al., 1988; Wilfley et al., 1993). However, Agras and colleagues (1997) found that those who stopped binge eating at posttreatment maintained their weight loss over the follow-up period, while those who continued to binge regained weight. Porzelius and colleagues (1995) found that 1- year after cognitive-behavioral therapy, women who were categorized as severe binge eaters at pretreatment continued to lose weight, while women who were categorized as non-binge eaters stopped losing weight.

To our knowledge, it has never been tested whether improvement in general well-being is related to weight loss in the long-term. In the present study, we predicted that improving psychological well-being and normalizing eating patterns of participants would finally be related to weight loss. It was hypothesized that: (1) at 1-year follow-up the cognitive therapy would be more effective than the behavioral therapy in improving psychological well-being (concerns about shape, weight, and eating, self-esteem and depression); (2) at 1-year follow-up, the improvement in psychological well-being might be related to weight loss.

\section{METHOD}

\section{Participants}

The selected participants were 74 obese women. They were recruited from a group of respondents answering local newspaper advertisements that offered two university-based treatments for eating problems. Inclusion criteria were: age between 18 and 50 years; and a BMI of 27 or higher. Exclusion criteria were: participation in a weight-loss program at the time of selection; current physical dependence on alcohol or drugs, psychosis; or pregnancy.

Obese women who responded to the advertisements were screened for the inclusion and exclusion criteria in a telephone interview. Interested and potentially eligible individuals $(\mathrm{N}=451)$ received the revised Questionnaire on Eating 
and Weight Pattern-revised (QEWP-r; Yanovski, 1993). This self-report questionnaire contains 28 items and is designed to collect information regarding demographics, weight history, dieting history, and psychiatric, and medical history. The questionnaire also contains items which specifically test for the Diagnostic and Statistical Manual of Mental Disorders (DSM-IV; American Psychiatric Association, 1994) criteria for binge eating disorder (BED). Furthermore, questions on inclusion and exclusion criteria were added. After completion at home, the questionnaires were returned to the university $(\mathrm{N}=265)$.

If respondents met the BED criteria or did not have any binge episodes at all, they $(\mathrm{N}=145)$ were invited for a structured diagnostic interview that lasted about 1 hour and was conducted by a psychologist experienced in the assessment of eating disorders and obesity. The interviewer clarified and checked the DSM-IV items, especially those regarding quantity of food and extent of loss of control and the exclusion criteria were checked once again. After the diagnostic interview, participants were weighed in street clothes, without shoes, on a balance beam scale and their height was measured. The purpose of the study was explained, and written informed consent for the treatment program was obtained.

Respondents were categorized as non-binge eaters if they fulfilled none of the DSM-IV criteria for BED. The BED diagnosis was made if respondents met strict DSM-IV criteria for the disorder. We anticipated that comparing two extreme groups would be most likely to reveal relevant differences. Therefore, the major reason for exclusion was if respondents met some of the DSM-IV criteria, but not all. Other individuals cancelled the interview appointment or did not appear at the interview, or decided not to participate in the program, primarily for practical reasons. Finally, 37 women who met the proposed DSM-IV criteria for BED and 37 non-binge eaters made up the definitive sample.

\section{Study Design}

An experimental design was chosen consisting of a pre- and a postmeasurement, and two follow-up measurements at 6-month and 1-year after treatment. Participants were recruited in two equal phases and were randomly assigned to one of the two treatment conditions, with binge status taken into account. Thus, this procedure resulted in four distinct groups: non-binge eaters who received behavioral therapy, binge eaters who received behavioral therapy, nonbinge eaters who received cognitive therapy, and binge eaters who received cognitive therapy.

Selected participants were asked to complete questionnaires at home before treatment, after treatment, and 6-month and 1-year after treatment. During an interview at the university, it was checked whether all items on the questionnaires had been filled in, frequency of overeating was measured with the help of a 28-day interview method (Fairburn \& Cooper, 1993) and participants' weight was obtained. 


\section{Treatment}

\section{General aspects}

Both treatments were given in groups and all groups met for 15 weekly sessions of 150 minutes each. Ten groups, each consisting of 7 or 8 participants were assisted by one therapist. Eight therapists, experienced in the behavioral and cognitive therapy of eating disorders and/or obesity, participated in this study, each facilitating the treatment of at least one group. If therapists' guided one treatment condition they were randomly assigned to $\mathrm{CT}$ or BT condition. If a therapist guided two treatment conditions they conducted a CT and BT group. For both conditions, detailed treatment protocols were developed. All therapists received an intensive training in the protocol. Weekly consultation sessions of approximately one hour with each therapist were held to ensure therapists' compliance with treatment protocols. The common features in both treatments were semi-structured and problem oriented, primarily concerned with the participants present and future rather than past, they provided a coherent treatment rationale, and they were self-monitoring and set homework assignments.

\section{Cognitive therapy (CT)}

The aim of this treatment was to change dysfunctional cognition's regarding shape, weight, eating, and dieting and underlying self-schemas with the help of the cognitive techniques outlined by Beck (1976). In the first session, the therapists explained the rationale of cognitive therapy. A binge/overeating circle was described as beginning with dysfunctional cognitions about shape, weight, eating, and dieting or negative self-schemas. Participants were instructed to selfmonitor situations that triggered binge eating or overeating, and participants had to describe their thoughts. Examination of this monitoring was an important focus of therapy. During the first half of the therapy session, the homework assignments of the participants were discussed. In the second half, participants practiced identifying and challenging dysfunctional cognitions and setting up behavioral experiments.

The first stage of the therapy (6 sessions), focussed on identifying and altering dysfunctional cognitions regarding shape, weight, and eating. The Socratic dialogue was used by the therapist to initialize active learning and to encourage a questioning, investigative attitude in the participants. Furthermore, participants were encouraged to engage in behavioral experiments designed to challenge their dysfunctional cognitions.

In the second stage (6 sessions), cognitive tech-niques directed at identifying and challenging negative self-schemas which perpetuated disordered eating were emphasized. Negative self-schemas were identified and challenged and behavioral experiments were also used. Examples of negative self-schemas were: 'If I go to the swimming pool, people will reject me' or 'If I gain weight, I will be worthless'.

The final stage ( 3 sessions) was largely concerned with the maintenance of progress following the end of treatment. Marlatt \& Gordon (1985) proposed 
that the Abstinence Violation Effect (AVE) accounts for some of the variability regarding relapse. The AVE model predicts that internal, stable and global attributions for a lapse increase the probability of overeating or binge eating. Participants were informed that lapses were normal and to be expected. The purpose of the cognitive restructuring procedure in the last phase was to reframe maladaptive attributions of causality regarding lapses, to make it easier to regain control.

\section{Behavioral therapy (BT)}

The aim of the behavioral therapy was to learn a healthy eating pattern by having three meals each day and three planned snacks, decreasing fat intake, eating about 1500-1800 kcal a day, increasing exercise habits, and recognizing and anticipating high-risk situations.

In the first session, the therapists explained the rationale of behavioral therapy. To regain control over eating it is important to learn a healthy eating pattern, without excessive restrictive dieting. Participants were told that treatment first needed to eliminate binge eating and overeating patterns by establishing regular, healthy eating patterns and that weight control needed to be a secondary concern.

Participants were told to self-monitor their food intake and eating patterns, binge episodes or episodes of overeating and the circumstances under which they occurred, such as time, presence of other people, and mood. Examination of the self-monitoring formed an important focus of therapy. In the first half of the session, homework assignments were discussed and in the second half, nutritional information was given. Furthermore, participants were advised to make gradual changes in eating habits and exercise levels. Therefore, weekly goals were set to change their eating habits, exercise level and general activity levels. The basic behavioral strategies were self-monitoring, goal-setting, and stimulus control techniques. Three forms of stimulus control techniques were used. First, participants learned stimulus-control techniques whereby stimuli which resulted in eating were avoided (e.g., not buying food, not going to a shop when hungry). Second, they learned techniques to anticipate high risk situations and to outline strategies to prevent eating, such as walking, talking with friends, or taking a shower. Third, participants learned self-reinforcement techniques, such as going to the movies or buying a magazine, and were positively reinforced by the therapist if they were not overeating. No attention was paid to dysfunctional cognitions.

\section{Dependent Variables}

Eating pathology was measured with the Eating Disorder Examination Questionnaire (EDE-Q; Fairburn \& Beglin, 1994). The EDE-Q is a 36-item selfreport questionnaire version of the Eating Disorder Examination investigatorbased interview, the so called Eating Disorder Examination (EDE; Fairburn \& 
Cooper, 1993). The EDE-Q is designed to assess the specific core psychopathology of eating disordered individuals and focuses on the previous 28 days. We used four subscales of the EDE-Q: Shape Concern, Weight Concern, Eating Concern, and Restraint. Wilfley, Schwartz, Spurrell, and Fairburn (1997) found modest to good agreement between the self-report version and the investigatorbased interview on these four subscales. However, they found little agreement when assessing binge eating. It was suggested that the performance of the EDE$\mathrm{Q}$ would be improved by clarifying the definitions of complex features, such as binge eating, with the help of an additional interview.

Binge eating was therefore assessed with the help of the interview method. Since Rossiter, Agras, Telch, \& Bruce (1992) proposed that recollecting the days with binge eating episodes is performed more accurately than recollecting the number of binge eating episodes, we measured the number of days in which an objective binge episode had occurred during the last 28-days.

Weight. Participants were weighed in street clothes, without shoes, on a balance beam scale.

Depression was measured with the Beck Depression Inventory (BDI; Beck, Ward, Mendelson, Mock, \& Erbaugh, 1961). The BDI is a 21-item self-report measure of severity of depressive symptomatology. The items are rated on a four-point scale ranging from 0 ('not depressed') to 3 ('severely depressed'), resulting in a range of scores from 0 to 63 . Higher scores indicate more severe depression.

Self-esteem was measured with the Rosenberg's Self-Esteem Scale (RSE; Rosenberg, 1965), a 10-item questionnaire measuring global self-esteem, with special reference to the self-evaluation of the individual regarding an attitude of approval or disapproval. Each item is scored on a four-point scale ranging from strongly agree to strongly disagree, resulting in a range from 0 to 40 . A lower score indicates more positive self-esteem.

\section{Statistical procedure}

The dependent variables were divided into eating pathology (dietary restraint and concerns about shape, weight, and eating), binge eating, weight, and generic psychopathology (depression and self-esteem). To investigate the longterm effectiveness of both treatments the results were analyzed with 2 (Treatment: BT versus CT) × 2 (Binge Status: obese binge eaters versus obese non-binge eaters) $\times 3$ (Time: pretreatment, 6-month and 1-year follow-up) repeated measures multivariate and univariate analyses of variance. If significant effects were found pairwise contrasts were contrasts were conducted comparing pretreatment and 1-year follow-up. In addition, to test whether there was relapse, pairwise contrast were conducted between 6-month and 1-year followup. Analyses were performed for the participants who completed treatment and excluding non-responders (completers' sample $(\mathrm{N}=60)$ ) and for participants who completed treatment including non-responders $(\mathrm{N}=64)$. 


\section{Drop-out during treatment and follow-up}

Ten (13.5\%) participants of a total of 74 participants dropped out of treatment ('treatment drop-outs'): three binge eaters and two non-binge eaters from the CT group and three binge eaters and two non-binge eaters from the BT group. Reasons that participants gave for dropping out were: not losing enough weight $(\mathrm{N}=4)$, travelling time $(\mathrm{N}=2)$, and disruptive life events $(\mathrm{N}=4)$. Drop-out rates did not differ between cognitive and behavioral therapy, or between binge eaters and non-binge eaters. If individuals declined to complete follow-up assessments they were considered as non-responders. At 6-month follow-up there were no non-responders. However, at 1-year follow-up, four women refused to take part in the assessment. All four women had received behavioral therapy and three of them were binge eaters. They agreed to a short telephone interview in which reasons for non-response and frequency of binge eating were explored. The reasons for non-response were that they were all disappointed in the behavioral therapy and were not motivated to fill in the questionnaires and to visit the university. The obese binge eaters had bulimic symptoms and the obese nonbinge eater reported a chaotic eating pattern and severe depressive symptoms. In total, $14(18.9 \%)$ participants of the 74 participants dropped out of treatment or were non-responders at 1-year follow-up. Three binge eaters and two non-binge eaters from the CT group and six binge eaters and three non-binge eaters from the BT group. T-tests were conducted on demographic variables and outcome measures at pretreatment (age, duration of obesity, duration of binge eating, weight, binge eating, eating concern, weight concern, shape concern, restraint, self-esteem and depression). Obese binge eaters who dropped out of treatment or were non-responders had a significantly lower self esteem, $\mathrm{t}(35)=2.33$, $\mathrm{p}<.05$, and more shape concern, $\mathrm{t}(35)=2.07, \mathrm{p}<.05$, than the other obese binge eaters. Obese non-binge eaters who dropped out of treatment or were non-responders were heavier, $\mathrm{t}(35)=2.09, \mathrm{p}<.05$, than the other obese nonbinge eaters. On all other variables, no differences were found. Participants who were non-responders or dropped out of behavioral therapy had a lower selfesteem, $\mathrm{t}(35)=2.25, \mathrm{p}<.05$, than the other participants in the behavioral therapy. Participants who were non-responders or dropped out of cognitive therapy also had a lower self-esteem, $\mathrm{t}(35)=2.16, \mathrm{p}<.05$, than the other participants in the cognitive therapy. On all other variables, no differences were found.

At pretreatment the mean age of the completer's sample was 38.6 years (SD = 6.6). The mean BMI was $33.0(\mathrm{SD}=4.2$ ), range between 27.0 and 45.2 . Seventy-seven percent of the sample was obese (BMI>30) and $23 \%$ of the sample had a BMI between 27 and 30. The mean BMI of participants in the behavioral therapy was 33.7 and in the cognitive therapy 32.4. Participants reported a mean duration of obesity of 21.2 years $(S D=8.5)$. Binge eating disorder patients reported a mean frequency of binge eating of 4 days per week $(\mathrm{SD}=1.7)$. The mean duration of their binge eating was 13.5 years $(\mathrm{SD}=6.5)$. 
Toble 6.1 Means and standord deviations at pretreatment and 6 month and I-year Follow. Up for all outcome measures

\begin{tabular}{|c|c|c|c|c|c|c|c|c|c|c|c|c|}
\hline \multirow{4}{*}{ Measure } & \multicolumn{12}{|c|}{ Behavioral therapy } \\
\hline & \multicolumn{6}{|c|}{ Non-binge eaters $(N=18)$} & \multicolumn{6}{|c|}{ Binge eaters $(N=10)$} \\
\hline & \multicolumn{2}{|c|}{ Pretest } & \multicolumn{2}{|c|}{ Follow-Up II } & \multicolumn{2}{|c|}{ Follow-Up-112 } & \multicolumn{2}{|c|}{ Pretest } & \multicolumn{2}{|c|}{ Follow-Up II } & \multicolumn{2}{|c|}{ Follow-Up- 112} \\
\hline & Mean & (SD) & Mean & (SD) & Mean & $(\mathrm{SD})$ & Mean & (SD) & Mean & $(S D)$ & Mean & (SD) \\
\hline$E D E-Q^{3}$ shape concern & 3.2 & $(0.9)$ & 2.6 & $(1.5)$ & 2.5 & $(1.5)$ & 5.0 & $(0.5)$ & 2.5 & (1.4) & 2.3 & (1.9) \\
\hline EDE-Q weight concern & 2.8 & $(0.8)$ & 2.1 & $(1.0)$ & 2.2 & $(1.2)$ & 4.0 & $(0.6)$ & 2.7 & (1.6) & 2.2 & $(1.9)$ \\
\hline EDE-Q eating concern & 1.2 & $(0.7)$ & 0.8 & $(0.9)$ & 0.5 & $(0.6)$ & 3.2 & $(0.8)$ & 1.1 & $(1.0)$ & 0.9 & $(0.9)$ \\
\hline EDE-Q restraint & 1.1 & $(0.9)$ & 1.7 & $(1.1)$ & 1.5 & $(0.9)$ & 1.6 & $(1.3)$ & 1.2 & $(1.0)$ & 1.0 & (1.1) \\
\hline Weight & 92.5 & $(9.8)$ & 89.8 & $(9.8)$ & 89.0 & $(10.5)$ & 100.7 & $(19.3)$ & 97.6 & (20.5) & 98.2 & $(20.4)$ \\
\hline Binge eating ( 28 days) & & & & & & & 16.7 & (7.2) & 1.3 & (3.1) & 1.4 & $(3.8)$ \\
\hline $\mathrm{BDI}^{4}$ & 7.7 & $(4.9)$ & 7.3 & $(6.3)$ & 7.2 & $(5.8)$ & 20.3 & $(10.2)$ & 7.1 & (3.8) & 7.9 & (6.2) \\
\hline RSEs & 19.5 & (5.3) & 19.1 & $(6.6)$ & 18.4 & (6.1) & 26.8 & (5.7) & 20.0 & (4.5) & 19.3 & (5.7) \\
\hline
\end{tabular}

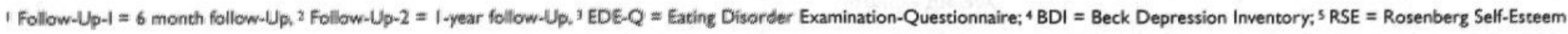
Scale (abile continues) 
Table 6.I Means and standard deviations at pretreatment and 6 month and 1-year Follow-Up for all outcome measures

\begin{tabular}{|c|c|c|c|c|c|c|c|c|c|c|c|c|}
\hline \multirow[t]{4}{*}{ Measure } & \multicolumn{12}{|c|}{ Cognitive therapy } \\
\hline & \multicolumn{6}{|c|}{ Non-binge eaters $(N=14)$} & \multicolumn{6}{|c|}{ Binge eaters $(N=18)$} \\
\hline & \multicolumn{2}{|c|}{ Pretest } & \multicolumn{2}{|c|}{ Follow-Up II } & \multicolumn{2}{|c|}{ Follow-Up-112 } & \multicolumn{2}{|c|}{ Pretest } & \multicolumn{2}{|c|}{ Follow-Up II } & \multicolumn{2}{|c|}{ Follow-Up- 112} \\
\hline & Mean & (SD) & Mean & $(\mathrm{SD})$ & Mean & (SD) & Mean & (SD) & Mean & (SD) & Mean & (SD) \\
\hline$E D E-Q^{3}$ shape concern & 3.4 & $(0.9)$ & 1.7 & $(0.9)$ & 1.4 & $(0.8)$ & 5.0 & $(0.5)$ & 2.6 & $(1.6)$ & 2.3 & $(1.3)$ \\
\hline EDE-Q weight concern & 2.8 & $(0.8)$ & 1.4 & $(0.8)$ & 1.2 & $(0.6)$ & 4.1 & $(0.6)$ & 2.0 & $(1.4)$ & 1.7 & (1.1) \\
\hline EDE-Q eating concern & 1.4 & $(0.5)$ & 0.5 & $(0.4)$ & 0.3 & $(0.4)$ & 3.5 & $(1.2)$ & 1.0 & $(1.1)$ & 0.9 & (1.1) \\
\hline EDE-Q restraint & 1.2 & $(1.0)$ & 0.9 & $(1.0)$ & 0.9 & $(1.0)$ & 1.5 & (1.2) & 1.3 & $(1.3)$ & 1.0 & (1.1) \\
\hline Weight & 86.0 & (8.7) & 85.5 & $(9.0)$ & 84.9 & $(10.5)$ & 94.7 & (15.7) & 95.4 & $(16.9)$ & 95.1 & $(16.8)$ \\
\hline Binge eating ( 28 days) & & & & & & & 16.1 & (7.0) & 0.2 & $(0.9)$ & 0.8 & (2.8) \\
\hline$\left.\mathrm{BD}\right|^{4}$ & 7.1 & (4.5) & 4.9 & $(4.0)$ & 3.9 & $(5.0)$ & 17.6 & $(5.2)$ & 8.2 & $(9.0)$ & 4.8 & (5.7) \\
\hline RSES $^{5}$ & 20.8 & $(5.0)$ & 16.6 & (5.1) & 18.2 & (6.9) & 26.6 & (3.2) & 21.2 & $(6.5)$ & 19.6 & (5.7) \\
\hline
\end{tabular}

I Follow-Up-I = 6 month Follow-Up, ${ }^{2}$ Follow-Up-2 = I-year Follow-Up, ${ }^{3}$ EDE-Q $=$ Eating Disorder Examination-Questionnaire; 4 BDI = Beck Depression Inventory; ${ }^{5}$ RSE $=$ Rosenberg SelfEsteem Scale 


\section{Treatment effects between pretreatment versus I-year follow-up and between 6-month and I-year follow-up}

\section{Dietary restraint and concerns about shape, weight, and eating}

Table 6.1 shows the means and standard deviations for all outcome measures at pretreatment, 6-month follow up and at 1-year follow-up for the completers' sample.

The repeated measures MANOVA performed on the pretreatment and followup scores on the four subscales of the EDE-Q showed a significant multivariate effect for Time, $\mathrm{F}(8,224)=15.96, \mathrm{p}<.001$. Univariate contrast analyses comparing pretreatment with 1 -year follow-up revealed that three of the four subscales showed significant Time effects $(\mathrm{p}<.001)$, indicating improvement on shape, weight, and eating concerns. There was no significant effect for restraint. Univariate contrast analyses between 6-month and 1-year follow-up yielded no significant Time effect, meaning that the positive results on the EDE-Q were maintained.

Furthermore, there was a trend for a multivariate Treatment $\mathrm{x}$ Time interaction, between pretreatment and 1-year follow-up, $\mathrm{F}(8,224)=1.52, \mathrm{p}=.015$. Additional univariate contrast analyses between pretreatment and 1-year followup revealed a significant Time $\mathrm{x}$ Treatment interaction for weight concern, $\mathrm{F}(1,57)=7.84, \mathrm{p}<.01$. For lasting positive effects on weight concern, the cognitive therapy proved to be more potent than the behavioral therapy. There were no significant Time $\mathrm{x}$ Treatment interactions for the subscales shape concern, eating concern, and restraint. Univariate contrast analyses between 6month follow-up and 1-year follow-up revealed no significant results.

Finally, there was a significant Time $x$ Binge Status effect between pretreatment and 1-year follow-up, $\mathrm{F}(8,224)=5.75, \mathrm{p}<.001$. Between pretreatment and 1 year follow-up three subscales revealed significant univariate Time $\mathrm{x}$ Binge Status interactions: shape concern, $\mathrm{F}(1,57)=12.36, \mathrm{p}<.001$; weight concern, $\mathrm{F}(1,57)$ $=10.75, \mathrm{p}<.01$; and eating concern, $\mathrm{F}(1,57)=27.94, \mathrm{p}<.001$. The restraint subscale showed a trend for a Time $\mathrm{x}$ Binge Status interaction, $\mathrm{F}(1,57)=3.71$, $\mathrm{p}=.06$. Inspection of the means revealed that binge eaters had improved more than non-binge eaters. Between 6-month follow-up and 1-year follow-up there were again no significant results, indicating that the results were maintained.

\section{Weight}

Results of the 2 (Treatment) x 2 (Binge Status) x 3 (Time) repeated measures ANOVA performed on the scores for weight yielded a significant effect for Time, $\mathrm{F}(2,114)=4.62, \mathrm{p}<.01$. Additional univariate contrast analyses showed a significant Time effect between pretreatment and 1-year follow-up, $F(1,57)=$ 5.92, $\mathrm{p}<.05$, and no significant Time effect between 6 -month and 1-year follow-up.

Furthermore, there was a trend for a Time $x$ Treatment interaction, $F(2,114)$ $=2.63, \mathrm{p}=.08$. Additional univariate contrast analyses between pretreatment and 1-year follow-up showed a marginally significant Time $\mathrm{x}$ Treatment effect, 
$\mathrm{F}(1,57)=3.59, \mathrm{p}=.06$, and no significant interaction effect between 6-month and 1-year follow-up. Participants in the behavioral therapy lost $3.0 \mathrm{~kg}$, while participants in the cognitive therapy lost $0.3 \mathrm{~kg}$. Finally, no significant effects for Time $\mathrm{x}$ Binge Status were found.

\section{Binge eating}

A 2 (Treatment) $\times 3$ (Time) repeated measures ANOVA using the number of objective binge episodes resulted in a significant main effect for Time, $F(2,52)$ $=109.91, \mathrm{p}<.001$. Additional univariate contrast analyses between pretreatment and 1-year follow-up showed a significant Time effect, $\mathrm{F}(1,26)=104.27$, $\mathrm{p}<.001$. There was no Time effect between 6-month and 1-year follow-up.

Finally, no significant interaction effect for Time $\mathrm{x}$ Treatment was found. Both treatments had a lasting impact on the reduction of binge eating. At 1-year follow-up the cognitive therapy had reduced bingeing by $95 \%$ and the behavioral therapy had reduced bingeing by $91 \%$. At 1 -year follow-up, $83 \%$ of the participants in the cognitive condition and $70 \%$ of the participants in the behavioral therapy abstained from binge eating. Furthermore, one obese binge eater in the behavioral therapy and one obese binge eater in the cognitive therapy fulfilled the DSM-IV criteria for binge eating disorder.

\section{Depression and self-esteem}

A 2 (Treatment) x 2 (Binge Status) x 3 (Time) MANOVA performed on the BDI and RSE showed a significant multivariate effect for Time, $\mathrm{F}(4,228)=17.65$, $\mathrm{p}<.001$. Univariate contrast analyses on the pretreatment and 1-year follow-up scores showed significant Time effects on depression, $\mathrm{F}(1,57)=75.24, \mathrm{p}<.001$ and self-esteem, $\mathrm{F}(1,57)=47.26, \mathrm{p}<.001$. Participants were less depressed and had a higher self-esteem between pretreatment and 1-year follow-up. Univariate contrast analyses between 6-month follow-up and 1-year follow-up showed a significant Time effect on depression, $F(1,57)=3.39, p=.07$, but not for selfesteem. Depression was still more improved between 6-month and 1-year follow-up, the results for self-esteem were maintained.

A multivariate marginally significant effect was found for Time $\mathrm{x}$ Treatment between pretreatment and 1 -year follow-up, $F(4,228)=1.87, p=.11$. Univariate contrast analyses showed a marginally significant effect on depression between 6-month follow-up and 1-year follow-up. No other significant Time $\mathrm{x}$ Treatment effects were found. The cognitive therapy was somewhat superior than the behavioral therapy in reducing depression between the 6-month and 1-year follow-up.

Finally, there was a multivariate interaction effect for Time x Binge Status, $\mathrm{F}(4,228)=12.17, \mathrm{p}<.001$. Additional univariate analyses between pretreatment and 1-year follow-up showed significant Time $\mathrm{x}$ Binge Status effects on depression, $F(1,57)=41.02, p<.001$ and self-esteem, $F(1,57)=16.62, p<.001$. No other significant Time $\mathrm{x}$ Binge Status effects were found. 
Ten participants dropped out of treatment and between 6-month follow-up and 1 -year follow-up, four participants in the behavioral therapy refused to participate in the 1-year follow-up assessment. The results presented so far might be influenced by selective drop-out. Therefore, additional end-point analyses were repeated on all outcome variables in which the missing values of the four treatment non-responders were replaced with the last scores available (6-month follow-up) ${ }^{5}$. There were differences between these end-point analyses and the analyses discussed earlier for the completers' sample. The repeated measures MANOVA performed on the pretreatment and 1-year follow-up scores on the four subscales of the EDE-Q showed a significant multivariate interaction effect for Time $\mathrm{x}$ Treatment, $\mathrm{F}(8,240)=2.30, \mathrm{p}=.02$. Additional univariate analyses revealed Treatment $x$ Time interactions for shape concern, $F(1,61)=5.95$, $\mathrm{p}<.01$; weight concern, $\mathrm{F}(1,61)=12.82, \mathrm{p}<.001$, and a trend for eating concern, $\mathrm{F}(1,61)=3.23, \mathrm{p}=.08$; there was no effect for restraint. Participants in the cognitive therapy showed a stronger reduction on these concerns than participants who received behavioral therapy between pretreatment and 1-year follow-up. A greater percentage of the participants in the cognitive condition were abstinent from binge eating $(83.3 \%)$, as compared to participants who received behavioral therapy $(53.8 \%)$. A Fisher exact test revealed a trend that the proportion of participants abstaining from binge eating at follow-up in the cognitive therapy was higher than in the behavioral group $(p=.08)$. It is notable that all three non-responders in the behavioral therapy still fulfilled the criteria for BED at 1-year follow-up. There were further no essential differences between the analyses including non-responders and the completers' analyses.

\section{Relationship between weight and psychological well-being}

Additional analyses were conducted with the completers' sample to determine whether those participants who lost weight showed more improvement on psychological variables than participants who gained weight. Participants were divided into two groups on the basis of weight loss between pretreatment and 1-year follow-up (participants who gained weight $(\mathrm{N}=24)$ and participants who lost weight $(\mathrm{N}=36)$. Success or failure at weight loss was used as a between group factor and time was used as a repeated measure in ANOVAs for shape concern, weight concern, eating concern, depression and self-esteem. We were interested in the interaction effects between psychological well-being and weight. The analyses showed no significant interaction effects between partici- 
Table 6.2 Means and standard deviations at pretreatment and I-year Follow-Up for participants who lost weight and regained weight on psychological outcome measures

\begin{tabular}{|c|c|c|c|c|c|c|c|c|}
\hline \multirow[b]{3}{*}{ Measures } & \multicolumn{4}{|c|}{$\begin{array}{l}\text { Participants who lost weight } \\
\qquad(\mathrm{N}=36)\end{array}$} & \multicolumn{4}{|c|}{$\begin{array}{l}\text { Participants who regained weight } \\
\qquad(N=24)\end{array}$} \\
\hline & \multicolumn{2}{|c|}{ Pretreatment } & \multicolumn{2}{|c|}{ I-year Follow-Up } & \multicolumn{2}{|c|}{ Pretreatment } & \multicolumn{2}{|c|}{ I-year Follow-Up } \\
\hline & Mean & (SD) & Mean & (SD) & Mean & (SD) & Mean & (SD) \\
\hline EDE-Q' shape concern & 3.9 & $(0.2)$ & 1.8 & $(0.2)$ & 4.3 & $(0.2)$ & 2.7 & $(0.3)$ \\
\hline EDE-Q weight concern & 3.2 & $(0.2)$ & 1.5 & $(0.2)$ & 3.7 & $(0.2)$ & 2.3 & $(0.2)$ \\
\hline EDE-Q eating concern & 2.1 & $(0.2)$ & 0.5 & $(0.1)$ & 2.5 & $(0.3)$ & 0.9 & $(0.2)$ \\
\hline$\left.\mathrm{BD}\right|^{2}$ & 10.4 & (1.3) & 4.7 & $(0.9)$ & 15.8 & (1.6) & 7.5 & (1.1) \\
\hline $\mathrm{RSE}^{3}$ & 22.6 & $(1.0)$ & 17.9 & $(1.0)$ & 24.0 & (1.8) & 20.3 & (1.2) \\
\hline
\end{tabular}

I $\mathrm{EDE}-\mathrm{Q}=$ Eating Disorder Examination-Questionnaire; ${ }^{2} \mathrm{BDI}=$ Beck Depression Inventory, ${ }^{3}$ RSE $=$ Rosenberg Self-Esteem scale

pants who gained weight and participants who lost weight between pretreatment and 1-year follow-up on psychological outcome measures. Table 6.2 presents the means and standard deviations on psychological well-being for participants who lost weight and gained weight.

\section{DISCUSSION}

The first hypothesis tested was that the cognitive therapy would be more effective than the behavioral therapy at improving psychological problems at 1-year follow-up. The results of this study showed that for obese binge eaters and obese non-binge eaters both treatments had a markedly positive and lasting impact on shape concern, weight concern and eating concern, binge eating, self-esteem and depression at 1-year follow-up. The cognitive and behavioral therapy were both equally successful on most outcome measures at 1-year follow-up. However, the results may be influenced by selective drop-out. Participants who did poorly in the behavioral therapy became non-responders at 1 -year follow-up. The analyses including non-responders showed that the cog- 
nitive therapy was superior in improving shape, weight, and eating concern and binge eating at 1-year follow-up. In addition, it was tested whether there was relapse between 6-month and 1-year follow-up. On almost all outcome measures the positive effects were maintained between 6-month and 1-year followup. Only for depression participants in the cognitive condition became significantly less depressed than participants in the behavioral therapy between 6month and 1-year follow-up.

The second hypothesis tested was that at 1-year follow-up an improvement in psychological well-being was related to weight loss. Shape concern, weight concern, eating concern, self-esteem, and depression were greatly improved between pretreatment and 1-year follow-up. However, participants who gained weight were as successful in changing psychological well being as participants who lost weight. This finding is similar to that reported by Marcus et al. (1988). We could not demonstrate that improvement in psychological well-being was related to weight loss at 1-year follow-up. In this study, most obese women had made several attempts at weight loss without long-term success, the women were unhappy with their figure and weight, were not severely obese, had poor selfesteem, a chaotic eating pattern, and were tired of dieting. Non-dieting treatments might be useful for obese persons who share these characteristics. Furthermore, for obese binge eaters both treatments had a lasting effect on reducing binge eating, and $70 \%$ (BT) and $83 \%$ (CT) were abstinent at 1-year follow-up. Further research will need to explore whether it is better to improve psychological well-being first, and whether weight loss should become a focus of treatment only after psychological well-being has improved or vice versa.

Little research has been done on the maintenance of change after non-dieting approaches to obesity. Primary outcome measures have mostly been frequency of binge eating and weight loss, and not attitudes to shape, weight, and eating. With regard to binge eating, our study showed that at 1 -year follow-up, $83 \%$ of the obese binge eaters in the cognitive condition and $70 \%$ of the binge eaters in the behavioral therapy abstained from binge eating. A study by Agras and colleagues (1997) found that 33\% abstained from binge eating 1-year after receiving cognitive-behavioral therapy. Furthermore, in this study we found that participants who received cognitive therapy had reduced bingeing by $95 \%$ and participants who received behavioral therapy had reduced bingeing by $91 \%$. Other studies, which evaluated the results of a cognitive-behavioral therapy at 1-year follow-up, found a reduction in binge eating of between $55 \%$ and $64 \%$ (Agras et al., 1997; Wilfley et al., 1993). The purely cognitive or behavioral therapy were at least as effective, if not more effective, than the multi-component cognitive-behavioral therapy. A possible explanation for this effect is that participants in unifaceted treatment packages learn and implement the techniques better than participants in more multifaceted treatment packages.

Obesity researchers have suggested that long-term weight losses of $10 \%$ of initial body weight are an appropriate treatment goal (Institute of Medicine, 1995). Bearing this in mind, long-term weight losses in our study were discouraging. Between pretreatment and 1-year follow-up, participants in the 
behavioral therapy lost $3.0 \mathrm{~kg}$, while participants in the cognitive therapy lost $0.3 \mathrm{~kg}$. The results are more or less comparable to weight losses achieved in some previous studies using the CBT program and other non-dieting behavioral programs of the same duration (Agras et al., 1997; Goodrick et al., 1998; Marcus et al., 1988; Wilfley et al, 1993). In a study by Porzelius and colleagues (1995), obese women with severe binge eating problems lost most weight in the cognitive-behavioral therapy and weight loss for obese women with no binge eating problem did not differ between the treatments at 1-year follow-up. In our study, we found no interaction effect for type of treatment and binge status.

In interpreting the results of this study, certain potential limitations of this study should be considered. A first limitation of this study was the lack of a no treatment control group, so the beneficial effects of this study may be due to a placebo effect. However, other controlled studies showed that behavioral treatments are superior to a no treatment control group (for a review see Wilfley \& Cohen, 1997). Furthermore, it is difficult to find a placebo control group that mimics the tested treatment in all ways, except the putative active factor (Omer \& London, 1989).

Obese binge eaters, as the most common eating disorder, and obese nonbinge eaters form a large group of potential health-care consumers. Carter and Fairburn (1998) mentioned that it is unlikely that there will be sufficient specialist resources to deal with the whole population of obese binge eaters and obese non-binge eaters. Therefore, it is important that specialists in primary and the community health care can also deal with obese binge eaters as well as obese non-binge eaters. It might be concluded from this study that an intensive cognitive intervention is not necessary for a subgroup of obese binge eaters and obese non-binge eaters as at 1-year follow-up the cognitive therapy and the behavioral therapy were equally effective on psychological well-being. However, there is also a great percentage of obese persons who did not respond to behavioral therapy. 


\author{
PRETREATMENT PATIENT CHARACTERISTICS AS PREDICTORS \\ OF ONE-YEAR OUTCOME AFTER TREATMENT FOR OBESITY \\ AND THE INFLUENCE OF SHAPE AND WEIGHT CONCERNS
}

\title{
Submitted for publication as:
}

Nauta, H., Hospers, H., \& Jansen, A. (2001). Pretreatment patient characteristics of oneyear outcome after treatment for obesity and the influence of shape and weight concerns.

\section{INTRODUCTION}

The problem of improving long-term outcome after treatment of obesity and eating disorders has received a great deal of attention by researchers. However, pretreatment factors that predict who will benefit from a given treatment at long-term follow-up have received little attention so far in literature (Wilfley \& Cohen, 1997). Understanding predictors of outcome could facilitate matching treatment to patients based on their pretreatment patient characteristics.

Across studies a number of general predictive factors have been identified that are related to treatment success: demographic variables, severity of symptoms, family history or axis I and axis II co-morbidity (Bulik, Sullivan, Joyce, Carter, \& McIntosh, 1998). Motivation for therapy is a predictor that has hardly been studied (Garfield, 1994). Individuals with obesity might be ambivalent about committing themselves to a lifelong regular and healthy eating pattern. Motivation might influence treatment outcome, with participants having the lowest motivation doing the least well (Vitousek \& Watson, 1998). Furthermore, a number of researchers have recently proposed that cognitive variables such as dysfunctional cognitions regarding shape and weight may play 
a crucial role in the etiology and maintenance of eating disorders and obesity. Until now, this line of research has received little empirical attention. A study of Fairburn and colleagues (1993) has shown that shape and weight concerns were significantly associated with treatment outcome in bulimia nervosa at 1year follow-up. Furthermore, they found that the residual shape and weight concerns at posttreatment predicted subsequent outcome.

The primary purpose of the present study was to examine pretreatment patient characteristics as predictors of 1-year outcome after treatment for obesity. Furthermore, we examined the central prediction of the cognitive view of obesity and eating disorders that the residual shape and weight concerns at posttreatment predict outcome at long-term follow-up (Fairburn et al., 1993).

\section{METHOD}

\section{Participants}

The data in this study are taken from a randomized clinical trial examining the efficacy of a cognitive and a behavioral therapy for obese binge eaters and obese non-binge eaters. Initial results from the clinical trial have been reported elsewhere (Nauta, Hospers, \& Jansen, 2001; Nauta, Hospers, Kok, \& Jansen, 2000). Selected participants were 74 obese women. They were recruited from a group of respondents who answered local newspaper advertisements that offered two university-based treatments for eating problems. Inclusion criteria were: age between 18 and 50 years; and a BMI of 27 or higher. Exclusion criteria were: participation in a weight-loss program at the time of selection, current physical dependence on alcohol or drugs, psychosis, or pregnancy.

Obese women who responded to the advertisements were screened in a telephone interview for the inclusion and exclusion criteria. Interested and potentially eligible individuals $(\mathrm{N}=451)$ received the Questionnaire on Eating and Weight Patterns-revised (QEWP-r; Yanovski, 1993). This self-report questionnaire contains 28 items and is designed to collect information regarding demographics, weight history, dieting history, psychiatric and medical history. The questionnaire also contains items which specifically test for the Diagnostic and Statistical Manual of Mental Disorders (DSM-IV; American Psychiatric Association, 1994) criteria for binge eating disorder (BED). Furthermore, questions on inclusion and exclusion criteria were added. After completion at home, the questionnaires were returned to the university $(\mathrm{N}=265)$.

If respondents met either all BED criteria or did not have any binge episodes at all, they $(\mathrm{N}=145)$ were invited for a structured diagnostic interview that lasted about 1 hour and was conducted by a psychologist experienced in the assessment of eating disorders and obesity. The interviewer clarified and checked 
the DSM-IV items, especially those regarding quantity of food and extent of loss of control and the exclusion criteria were checked again. After the diagnostic interview, participants were weighed in street clothes, without shoes, on a balance beam scale and their height was measured. The purpose of the study was explained, and written informed consent for the treatment study was obtained.

Respondents were categorized as non-binge eaters if they fulfilled none of the DSM-IV criteria for BED. The BED diagnosis was made if respondents met strict DSM-IV criteria for the disorder. Finally, 37 women who met the proposed DSM-IV criteria for BED and 37 non-binge eaters comprised the sample. Participants were recruited in two equal phases and were randomly assigned to one of the two treatment conditions, taking binge status into account. In total, five cognitive groups and five behavioral groups were constructed each containing 7 or 8 participants. Twenty-one obese persons with BED received cognitive therapy and sixteen received behavioral therapy. Sixteen obese non-binge eaters received cognitive therapy and twenty-one received behavioral therapy.

Ten participants $(13.5 \%)$ of a total of 74 participants dropped out of treatment: 3 binge eaters and 2 non-binge eaters from cognitive therapy and 3 binge eaters and 2 non-binge eaters from the behavioral therapy. Dropout rates did not differ between cognitive and behavioral therapy, nor between binge eaters and non-binge eaters. Analyses were conducted on the sample of 64 treatment completers. Four of the 64 treatment completers completed the 6month follow-up, but refused to take part at the 1-year follow-up. Therefore, the missing values of these four persons were replaced with the last scores available (6-month follow-up). At pretreatment the mean BMI was 33.0 (SD = 4.1 ), ranging from 27.0 to 45.2 .

\section{Study design}

Participants who met for 15 weekly sessions of 150 minutes cognitive or behavioral therapy for obesity were asked to complete questionnaires at home before treatment, after treatment, and at 1-year follow-up. During an interview at the university, it was checked whether all items on the questionnaires had been completed, frequency of overeating was measured with the help of a 28day interview method (Fairburn \& Cooper, 1993) and participants' weight was obtained.

\section{Measures}

\section{Outcome measures}

Two measures of 1-year outcome were used, because obesity is multifaceted and there is no single treatment outcome for obesity.

Eating pathology, as measured with the Eating Disorder ExaminationQuestionnaire (EDE-Q; Fairburn \& Beglin, 1994), was the first outcome mea- 
sure. The EDE-Q is a 36-item self-report questionnaire version of the Eating Disorder Examination investigator-based interview, the so called Eating Disorder Examination (EDE; Fairburn \& Cooper, 1993). The EDE-Q is designed to assess the specific core psychopathology of eating disordered individuals and focuses on the past 28 days. We used four subscales of the EDE-Q: Shape Concern, Weight Concern, Eating Concern, and Restraint. Wilfley, Schwartz, Spurrell, and Fairburn (1997) found modest to good agreement between the self-report version and the investigator-based interview on these four subscales.

Body Mass Index (BMI), as measured as weight in kilograms and height in squared meters, was the second outcome measure. Participants were weighed in street clothes, without shoes, on a balance beam scale.

\section{Predictor measures}

Predictors were divided into five categories: demographic variables, severity of symptoms, family history of obesity, motivation and cognitive variables. The aim was to attempt as broad as a range of measures without overloading the analysis with possible redundant outcome measures or dependent measures used in the outcome study (e.g. depression and self-esteem). The measures of each category will be described below.

Demographic variables. Participants age (years) and level of education were recorded. Level of education was categorized as low, medium or high. The low level referred to vocational training, the medium level to advanced vocational training and the high level to college/ university training.

Severity of symptoms. Includes time on a diet (years), the duration of obesity (years) and if persons were binge eating disorder patient ('yes or no').

Family history of obesity. It was measured with the question 'Are your parents obese?'

Motivation. At the start of treatment participants rated the extend to which they were motivated for therapy on a Visual Analogue Scale (VAS), ranging 102 from 0 'not at all' to 100 'very much'.

Cognitive variables. These variables were measured at pre- and posttreatment as the sum of two EDE-Q items that directly address the shape and weight concerns, 'importance of shape' and 'importance of weight' in line with the study of Fairburn et al. (1993). Each item is scored on a six-point scale ranging from 0 'not at all' to 6 'very much'. A higher score indicates more shape and weight concerns. The posttreatment level of shape and weight concerns was chosen to test if residual level of shape and weight concerns predicts 1-year outcome after treatment. 


\section{Characteristics}

Obese treatment completers $(\mathrm{N}=64)$

\section{Education (\%)}

Low

Medium

High

Binge status (\%)

Non-binge eaters

Binge Eating Disorder

Time on dieting in life (\%)

None or almost none

Quarter of the time

Half the time

Three-quarter of the time

Almost all the time

Family history of obesity (\%)

Yes

No

Age

Duration of obesity

Motivation

Shape and weight concerns
51.6

20.3

26.6

18.8

56.3

25.0

48.4

20.3

76.6

23.4

$38.6 \pm 6.6$

$21.1 \pm 8.3$

$89.0 \pm 10.9$

$4.5 \pm 1.1$

Values are percentages or $M \pm S D$

\subsection{RESULTS}

Demographic variables, severity of symptoms, family history of obesity, motivation and shape and weight concerns of obese participants at pretreatment are displayed in Table 7.1. At pretreatment the mean age of the treatment completers' was 38.6 years. Most participants received a medium level of education (56.3\%). About half of the participants reported binge episodes with a mean frequency of four binge episodes a week. Most participants reported a time on dieting of half a lifetime or more, and a mean duration of obesity of 21 year. Seventy-seven percent reported a family history of obesity. The participants were highly motivated, and reported a high level of shape and weight concerns $(\mathrm{M}=4.5, \mathrm{SD}=1.1)$. 
Table 7.2 Regression analyses of pretreatment predictor variables and eating disorder pathology and BMI at I-year ofter treatment

\begin{tabular}{lll}
\hline Variables & $\begin{array}{c}\text { Eating disorder pathology } \\
\text { Beta }\end{array}$ & $\begin{array}{l}\text { BMI } \\
\text { Beta }\end{array}$ \\
\hline Treatment condition & $-.28^{\text {*** }}$ & -.15 \\
Age & - & - \\
Education & - & - \\
Binge Eating Disorder & - & $.30^{* * *}$ \\
Duration of obesity & - & - \\
Time on a diet & - & - \\
Family history of obesity & - & $.27^{* * *}$ \\
Motivation & $-.20^{* *}$ & - \\
Shape and weight concerns & & $.14^{* *}$ \\
(pretreatment) & & $.14^{* * * *}$ \\
R & .43 & .18 \\
R2 & $4.51^{* * * *}$ & \\
F & & .41 \\
\hline
\end{tabular}

"p<.10, two-tailed, ** $p<.05$, two-tailed, ${ }^{* * *} p<.01$, two-tailed

- variable excluded in stepwise regression

\section{Pretreatment patient characteristics as predictors of I-year outcome}

104 Stepwise multiple regression analyses were performed using each of the predictor variables mentioned above to establish their unique contribution to overall eating disorder pathology at 1 -year after treatment. To control for differences in treatment condition, this variable was entered first. Stepwise forward regression analysis revealed that besides treatment condition two factors were related to eating pathology at 1-year follow-up. Shape and weight concerns were found to be positively related to eating disorder pathology $(B=.24)$. Motivation was found to be negatively related to eating disorder pathology at 1-year follow-up $(B=-.20)$. These factors contributed for $18 \%$ of the explained variance. $A$ second regression analysis was performed with the predictor variables to establish their contribution to BMI at 1-year follow-up. It was shown that the diagnosis binge eating disorder at pretreatment $(B=.30)$ and a family history of obesity $(B=.27)$ were positive related to BMI at 1 -year follow-up. These factors accounted for $17 \%$ of the explained variance. Table 7.2 summarizes these results. 


\begin{tabular}{lll}
\hline Variables & $\begin{array}{c}\text { Eating disorder pathology } \\
\text { Beta }\end{array}$ & $\begin{array}{c}\text { BMI } \\
\text { Beta }\end{array}$ \\
\hline Treatment condition & $-.20^{*}$ & -.02 \\
Shape and weight concerns & & \\
(posttreatment) & $.30^{\text {*10k }}$ & .13 \\
R & & .13 \\
$R^{2}$ & .40 & .02 \\
$F$ & .16 & .54 \\
\hline
\end{tabular}

${ }^{*} p<, 10$, two-tailed, $* *$ p $<.05$, two-tailed, *** $p<.01$, two-tailed.

\section{Shape and weight concerns at posttreatment as predictors of I-year outcome}

The second question examined if the level of shape and weight concerns (cognitive disturbance) at posttreatment will predict outcome 1-year follow-up. Stepwise regression analysis revealed that besides treatment condition, a high level of shape and weight concerns at posttreatment were found to be highly positive related to eating disorder pathology at 1-year follow-up. A second regression analysis revealed that a high level of shape and weight concerns at posttreatment were not related to BMI at 1-year follow-up. See Table 7.3.

\section{DISCUSSION}

The first aim of this study was to examine pretreatment predictors of outcome 1-year following short-term behavioral or cognitive therapy for obesity. We chose a definition of outcome anchored in psychological factors (shape and weight concerns) and BMI. It was found that less shape and weight concerns and a high motivation at pretreatment were predictive of less severe eating disorder pathology at 1-year follow-up. The diagnosis BED at pretreatment and a family history of obesity were predictive of a higher BMI at 1-year follow-up. The second aim of this study was to examine if shape and weight concerns at posttreatment were predictors of outcome at 1-year follow-up It was found that less cognitive disturbance directly after treatment predicted less severe eating 
pathology at 1-year follow-up. However, the level of cognitive disturbance directly after treatment did not emerge as a significant predictor of BMI at 1year follow-up.

An important finding of this study was that less concerns about shape and weight at pretreatment was a significant predictor of less eating pathology at 1year follow-up. This finding is in accord with clinical findings, but is in contradiction to the findings of Fairburn et al. (1993), who reported that those having the most severe concerns about shape and weight had the least severe eating pathology at 1-year follow-up. However, Fairburn and colleagues mentioned that they could not explain their result and that is was not according their intuition. Furthermore, we found that less concerns about shape and weight at posttreatment was a predictor of better outcome on eating pathology at 1-year follow-up. This finding confirms the results of the study from Fairburn et al. (1993) on this topic. These data may be viewed as support for the cognitive view of eating disorders and obesity.

Motivation was a significant predictor of treatment outcome at 1-year followup. This finding is consistent with other studies using cognitive-behavioral techniques for a sample of Obsessive Compulsive Disorder patients (De Haan et al., 1997), a sample of panic disorder patients (Keijsers, Hoogduin, \& Schaap, 1994), alcohol dependent patients (Guydish \& Greenfield, 1990; Kristenson, 1990) and chronic pain (Gale \& Funck, 1984). Furthermore, in a worksite weight control program was also found that motivation to change early in treatment was the best predictor for success (Prochaska, Norcross, Fowler, Follick, \& Abrams, 1992). In light of these findings motivational enhancement techniques (Treasure \& Ward, 1997; Vitousek, Watson, \& Wilson, 1998) at the start of treatment will be an important treatment strategy. Motivational enhancement is grounded on the transtheoretical model of behavior change (Prochaska \& DiClemente, 1983; DiClemente \& Prochaska, 1998). This model offers an integrative framework for understanding and intervening with human intentional behavior change. The model characterizes a series of five stages through which persons move if they successfully change their behavior ranging from no readiness to change towards action and maintenance. A behavior change will only begin if a person is in the action stage and has made a commitment to change.

A family history of obesity was found to be a predictor of a higher BMI at 1year follow-up. This is in accordance with literature in which the strongest predisposing factor for the development and maintenance of obesity appears to be family history, probably mediated by genetic predisposition rather than environment (Bouchard, Perusse, Leblanc, Tremblay, \& Theriault, 1988; Bouchard \& Perusse, 1993). Besides the genetic contribution to weight, binge eating was also a predictor of a higher BMI. This is in line with other studies that showed that obese binge eaters lose significantly less weight or rapidly regain it (Keefe, Wyshogrod, Weinberger, \& Agras, 1984; Marcus, Wing, \& Hopkins, 1988; Yanovski, Gormally, Leser, Gwirtsman, \& Yanovski, 1994). Research has shown that cognitive or behavioral therapy seem to be effective in reducing binge eating in obese binge eaters (Agras et al., 1994; Marcus, Wing, \& Fairburn, 
1995; Smith, Marcus, \& Kaye, 1992; Telch, Agras, Rossiter, Willey, \& Kenardy, 1990; Wilfley et al., 1993).

The results must be interpreted with several caveats in mind. First, these analyses focused only on those individuals who completed treatment. Of the participants who dropped out of treatment we had no 1-year follow-up data. Therefore, these predictors have no bearing on determining who is likely to remain in treatment versus who will drop out treatment. Second, our treatment sample was too small to examine specific predictors of outcome for obese binge eaters and obese non-binge eaters separately.

In conclusion, the results indicate that in individuals with obesity less shape and weight concerns and a high motivation are predictors for successful outcome on eating pathology at 1 -year follow-up. The diagnosis BED and a family history of obesity were predictive of a higher BMI at 1-year follow-up. This study therefore suggests the need to address attention to these factors in (cognitive)-behavioral therapy for obesity. 
The research presented in the current dissertation focused on the effectiveness of a cognitive and a behavioral therapy for obese binge eaters and obese nonbinge eaters. In Chapter 1 the psychological heterogeneity of obese persons was addressed and two subgroups, persons with and without BED, were introduced. In the next two Chapters a cognitive-behavioral model for obesity and BED was given and a detailed description was provided of the cognitive and behavioral protocols developed for the research addressed in this thesis. The subsequent empirical Chapter tested differences in frequency and content of cognitions between obese non-binge eaters and persons with BED. In the next three Chapters, the effectiveness of the cognitive and behavioral therapy at short and long-term follow-up was investigated and predictors for long-term outcome were sought. In the present and final Chapter the main empirical findings reported in the previous Chapters will be briefly summarized and discussed. Furthermore, the methodological strengths and limitations will be addressed. Finally, suggestions for future research and clinical practice will be given. 


\section{SUMMARY AND DISCUSSION OF THE MAIN CONCLUSIONS}

In the present paragraph the main conclusions of the empirical studies will be summarized. Several conclusions can be made about the centrality of shape and weight concerns. It was found that negative self-schemas, which reflect negative self-evaluations influenced by shape, weight, or eating, were more frequently found in obese binge eaters than in obese non-binge eaters. Obese binge eaters also believed their negative self-schemas and shape, weight and, eating concerns to a greater extent than obese non-binge eaters (Chapter 4). In addition, it was found that less shape and weight concerns directly after treatment were predictors of less severe eating pathology at 1-year follow-up (Chapter 7).

The results of the treatment evaluation study showed that at short and longterm follow-up the cognitive and behavioral therapy were both effective. However, the cognitive therapy was more effective in reducing a broad spectrum of complaints than the behavioral therapy. Obese binge eaters benefited more from treatment than obese non-binge eaters (Chapter 5 and 6).

\section{Shape and weight concerns}

Of primary importance in the proposed model for obesity and BED is the value attached to body weight and shape. In persons with eating disorders shape and weight concerns are characteristic and they evaluate their self-worth primarily in terms of body shape and weight (Fairburn et al., 1993; Jansen, 1998; Vitousek \& Hollon, 1990). The present thesis supports the idea that this is also true for obesity. In Chapter 5, it was found that obese binge eaters as well as obese non-binge eaters showed high concerns about body weight and shape. In addition, obese binge eaters scored significantly higher on the weight, shape and eating concerns subscales of the EDE-Q than obese non-binge eaters. In 110 addition, in Chapter 4 it was shown that the majority of obese binge eaters mentioned negative self-schemas that could be characterized as negative generalizations about the self combined with weight, shape, or eating concerns. The majority of obese non-binge eaters mentioned weight, shape, and eating concerns which were not combined with negative generalizations about the self. Participants with negative self-schemas, irrespective of binge category, were more depressed and had lower self-esteem than the other participants. Finally, with respect to the content analyses of negative self-schemas, it was found that both groups most often mentioned themes such as rejection, unworthiness and lack of willpower. However, obese non-binge eaters mentioned more selfschemas regarding lack of willpower, whereas obese binge eaters were more preoccupied with rejection and unworthiness. Chapter 7 showed that less concerns about shape and weight at pretreatment and posttreatment was a significant predictor of less eating pathology at 1-year follow-up.

Persons with BED can be distinguished as a distant subgroup among the obese. Binge eating disorder is characterized by a pattern of regular and sus- 
tained binge eating. Furthermore, persons with BED showed more severe concerns about shape, weight, and eating than obese non-binge eaters. In addition, it was confirmed that obese binge eaters reported more severe depression and a lower self-esteem than obese non-binge eaters. It seems that an effective treatment for binge eating disorder must include elimination of binge eating, shape and weight concerns, and depressive symptomatology.

It is important that cognitions regarding body weight and shape in obese persons are addressed. The data suggest that cognitive therapy might be useful in the treatment of obese binge eaters and obese non-binge eaters. Apart from challenging dysfunctional cognitions about weight, shape, and eating, it is necessary to address negative self-schemas. In case of obese binge eaters, one should be mindful of negative self-schemas regarding rejection and unworthiness, whereas in case of obese non-binge eaters negative self-schemas concerning lack of willpower might be relevant.

What is the role of shape and weight concerns in the development and maintenance of disturbed eating and weight control behaviors in obesity? It was beyond the scope of this thesis to test all hypotheses that can be derived from the cognitive model for obesity and binge eating disorder. For further research it would be valuable to test them empirically. For example: Does a negative mood, triggered by shape and weight concerns, lead to overeating or binge eating; or do shape and weight concerns lead to poor eating control?

\section{Short and long-term effectiveness of cognitive and behavioral therapy}

The studies described in Chapter 5 and 6 were concerned with the effectiveness of a cognitive and behavioral therapy for obese binge eaters and obese nonbinge eaters. The cognitive therapy was expected to produce greater changes in psychological well-being (depression and self-esteem) and dysfunctional cognitions, although in the short term weight loss was expected to be greater in the behavioral therapy. The results showed that cognitive and behavioral therapy were both very effective in reducing a broad spectrum of complaints. However, the cognitive therapy as compared to the behavioral therapy was more effective in reducing concerns about shape, weight, and eating, and led to improved selfesteem. The behavioral therapy as compared to the cognitive therapy was more effective in reducing weight. At 6-month and 1-year follow-up, the cognitive and behavioral therapy remained both effective on a broad spectrum of outcome measures. However, participants in the cognitive therapy showed more reductions in shape, weight, and eating concerns and a greater percentage of participants were abstained from binge eating, than participants in the behavioral therapy. The long-term results for weight loss were disappointing. Between pretreatment and 1-year follow-up participants in the behavioral therapy lost $3.0 \mathrm{~kg}$, whereas participants in the cognitive therapy lost $0.3 \mathrm{~kg}$. However, participants who gained weight between pretreatment and 1-year follow-up were as successful in changing psychological well-being as participants who lost weight. 
In this study significant reductions were seen on the shape, weight, and eating concerns subscales of the EDE-Q. The reductions were both statistically and clinically significant. According to Fairburn and colleagues (1993), a clinically significant change on the EDE can be operationalized as whether participants meet scores within one standard deviation of the scores obtained from persons who do not seek treatment. Telch and Stice (1998) report these normative data on the EDE-Q for sixty non-treatment seeking obese persons. In the Telch and Stice (1998) study, it was found that at posttreatment and follow-up the mean scores on shape, weight, and eating concerns of obese binge eaters and obese non-binge eaters were within one standard deviation of the scores obtained from the obese persons who did not seek treatment. Furthermore, significant improvements on depression and self-esteem were found. According to the Center for Cognitive Therapy the following guidelines for BDI cut-off scores can be made; no to minimal depression is $<10$, mild to moderate depression is 10-18, and moderate to severe depression is 19-29 (Beck, Steer, \& Garbin, 1988). At pretreatment the level of depression in obese binge eaters was moderate to severe (mean BDI score $=19.3$ ), whereas the level of depression in obese non-binge eaters was minimal (mean BDI score $=7.9$ ). At posttreatment and follow-up a clinically significant improvement on the BDI was demonstrated. The mean depression score of obese binge eaters and obese non-binge eaters fall within the no to minimal range of depression or in the lowest range of the mild to moderate depression. The binge abstinence rates, particularly in the cognitive intervention, were high. After treatment, $67 \%$ of obese binge eaters abstained from binge eating in the cognitive therapy, compared to $44 \%$ abstinence in the behavioral therapy. At follow-up $86 \%$ of the participants in the cognitive condition were abstinent from binge eating and $44 \%$ of the obese binge eaters in the behavioral therapy. Data from trials administering CBT suggest abstinence rates in obese binge eaters ranging from 28\% to 79\% (Agras et al., 1994; Agras et al., 1995; Marcus et al., 1995; Smith et al., 1992; Telch et II2 al., 1990; Wilfley et al., 1993), with a mean abstinence rate of approximately $50 \%$.

In contrast to improvements in eating, weight, and shape concerns, psychological well-being, and binge eating, the results for weight loss were disappointing. It was found that at 1-year follow-up participants in the behavioral therapy showed a mean weight loss of $3.0 \mathrm{~kg}$, while participants in the cognitive therapy showed a mean weight loss $0.3 \mathrm{~kg}$. In a review, it was found that between 1988 and 1990 traditional behavioral treatment programs at follow-up had a mean weight loss percentage of $6 \%$ (Wadden \& Bartlett, 1992). In addition, at follow-up many more studies showed that almost all participants return to their baseline weights or were eventually even weighing more than they did at the start of treatment (e.g., Garner \& Wooley, 1991; Jeffery et al., 2000; Perri et al., 1987; Perri, Nezu, Patti, \& McCann, 1989; Wilson, 1994; Wooley \& Garner, 1991). It may be necessary to examine how much weight obese persons with and without binge eating problems can reasonably lose and maintain. Benefits of modest weight losses have been ignored for a long time. There is 
now considerable evidence that small weight losses $(10 \%)$ can produce medical benefits (Institute of Medicine, 1995). The possibility also exists that smaller losses will be more easily maintained for at least 1-year, whereas larger losses are not (Brownell \& Wadden, 1992). Nevertheless, these small weight losses seem hard to maintain. Given the notorious difficulty of losing weight and the maintenance of weight loss, it may be more realistic to give more emphasis to non-dieting approaches which focus on psychological well-being, healthy body related cognitions, and a healthy eating pattern. These programs do prevent weight gain and even some small weight losses are achieved which may be a success in its own right. Besides, in a population-based prospective study of 5115 young adults initially aged 18-30 years, it was found that among white women the average weight gain over 10 -years was approximately $0.55 \mathrm{~kg}$ per year (Lewis et al., 2000). These data indicate that young adults are at risk for weight gain if not treated.

An intriguing aspect of the evaluation study was that, despite success in reducing binge eating, both treatments have failed to produce consistent weight loss in obese BED participants. The results are comparable to previous studies using a CBT program for persons with BED (Agras et al., 1994; Agras et al., 1995; Agras et al., 1997; Goodrick et al., 1998; Marcus et al., 1995; Telch et al., 1990; Wilfley et al, 1993). A possible explanation for this result is that the total energy intake remained the same. Future research should examine the dietary intake of participants with help of an eating diary during the assessment periods. However, research has shown that obese persons frequently underreport their dietary intake, particularly fatty foods or foods rich in carbohydrates (Heitmann \& Lissner, 1995). So, it is difficult to obtain valid information on dietary intake. Methods such as the doubly labeled water technique have been used to describe the amount of underreporting (e.g., Westerterp, 1999). The doubly labeled water technique estimates energy expenditure under normal living conditions and when obese persons are in energy balance the energy intake is equal to the energy expenditure.

This has been the first study with obesity and BED in which the cognitive and behavioral component of treatment were separated. Therefore, future research should indicate if the results can be replicated. In addition, future research should also examine if the full package of cognitive-behavioral therapy as compared to a cognitive and behavioral therapy contributes to an even better outcome. Furthermore, both short and long-term effects should be examined. Especially the long-term outcome would be valuable. A problem with long-term outcome is that it is difficult to control for possible confounding variables or that participants are not motivated to come to the assessment.

The finding that cognitive therapy was more effective in reducing shape, weight and eating concerns than the behavioral therapy supported the cognitive model for obesity and BED. Cognitive techniques directly trigger dysfunctional cognitions. Interestingly, also the behavioral therapy led to significant changes in shape, weight, and eating concerns. It might be that participants through changing their eating behavior experienced disconfirming information. For 
example, if an obese woman in the behavioral condition experienced that she could maintain a regular eating pattern, the cognition 'I have no control over eating' was disconfirmed in an indirect way.

\section{METHODOLOGICAL STRENGTHS AND LIMITATIONS}

Some issues concerning the internal and external validity of our randomized trial will be discussed. Internal validity refers to the degree to which changes in dependent variables can be truly attributed to the experimental manipulations. External validity refers to the generalization of the results of the trial to the general population

\section{Study Population}

Participants were recruited from a group of respondents answering to local newspaper advertisements that offered two university-based treatments for eating problems. Binge eating disorder diagnosis was made through the use of a clinical interview based on the most recent DSM-IV criteria. The advantage of this measurement is that it presents a widely used and standardized method. In many ways, obese binge eating women who participated in this study were comparable on levels of frequency of binge eating to samples of obese binge eaters in other countries, merely the United Kingdom and the United States (e.g., Carter \& Fairburn, 1998; Wilfley et al., 1993).

The generalization of the results for all obese women seeking treatment 114 might be limited. Our sample of obese women had many experiences with dieting programs and most were dissatisfied with dieting and wanted a different treatment for their eating problem than another dieting program. For practice it should be advisable to give obese persons with a long dieting history a more realistic view about the effectiveness of dieting approaches. Obese persons should know that obesity is now considered as a chronic condition (e.g., Wilson, 1999).

Ten participants (13.5\%) of a total of 74 participants dropped out of treatment: 3 binge eaters and 2 non-binge eaters from the CT group and 3 binge eaters and 2 non-binge eaters from the BT group. Reasons that participants gave for dropping out were: not losing enough weight $(\mathrm{N}=4)$, traveling time $(\mathrm{N}=$ $2)$, and disrupting life-events $(\mathrm{N}=4)$. Dropout rates did not differ between cognitive and behavioral therapy, or between binge eaters and non-binge eaters. Drop-out rates after cognitive-behavioral therapy range between $8 \%$ to $20 \%$ in obese participants and between $11 \%$ to $33 \%$ in participants with BED (see Chapter 2). The drop-out rate in our study was reasonable. At 1-year follow-up, 
four women refused to take part in the assessment. All four women had received behavioral therapy and three of them were binge eaters at posttreatment. The reasons for non-response were that they were all disappointed in the behavioral therapy. Because this might be the result of selective drop-out, analyses were performed for participants who completed treatment and excluding non-responders and for participants who completed treatment including non-responders.

\section{Interventions}

In both treatment protocols special attention was given to treatment integrity. Once treatment starts it is essential to ensure that treatment is carried out as intended. All therapists' had experience in the treatment of eating disorders or obesity and they were given a training to learn to carry out the techniques before treatment began. In addition, detailed treatment manuals were written. Finally, weekly supervision sessions were held.

Furthermore, manipulation checks were conducted. Manipulation checks have been hardly applied in psychotherapy outcome research. During treatment dysfunctional cognitions and eating behavior were measured three times to determine their change during treatment. The cognitive checklist contained each participant's most prominent dysfunctional cognitions regarding shape, weight, and eating that were identified in a semi-structured face-to-face interview before treatment. The eating checklist measured regularity of eating during the previous seven days. It was intended that participants in the cognitive therapy showed a reduction in dysfunctional cognitions, whereas participants in the behavioral therapy showed a more regular eating pattern. In Chapter 5, it was found that participants in the cognitive condition reduced their degree of belief in dysfunctional cognitions to a greater extent than participants in the behavioral condition. The eating pattern became more regular for participants in the behavioral condition than for participants in the cognitive condition. Thus the interventions were carried out as intended. By doing so, the process of treatment can be determined and this might be a critical step in evaluating theory about the basis of particular treatment techniques.

In our study the results of a cognitive and a behavioral therapy were directly compared with each other. A potential limitation of the study design was the lack of a no treatment control group or a placebo control condition, so the positive effects might be due to placebo effects. Nevertheless, six controlled studies showed that cognitive-behavioral therapy is superior to a no treatment control group (Agras et al., 1995; Marcus et al., 1995; Eldredge et al., 1997; Peterson et al., 1998; Telch et al., 1990; Wilfley et al., 1993). Furthermore, it is very difficult to design a placebo condition in which the experimental treatment is imitated in all ways, except the specific active factor (Omer \& London, 1989). In addition, placebo groups are, in essence, therapy groups and effective ones often include many of the same ingredients as treatment groups (Lambert, Shapiro, \& Bergin, 1986). 


\section{Outcome measures}

The Eating Disorder Examination-Questionnaire (EDE-Q; Fairburn \& Beglin, 1994), a standardized measurement, was chosen to measure eating pathology. The EDE-Q is designed to assess the specific core psychopathology of eating disordered individuals and focuses on the past 28 days. We used four subscales of the EDE-Q: shape concern, weight concern, eating concern, and restraint. Wilfley and colleagues (1997) and Luce \& Crowther (1999) found modest to good agreement between the self-report version and the investigator-based interview on these four subscales. However, they found low agreement when assessing binge eating. It was suggested that the performance of the EDE-Q would improve by clarifying the definitions of complex features, such as binge eating, with the help of an additional interview. Therefore, binge eating was assessed with the help of the interview method. Since Rossiter, Agras, Telch, \& Bruce (1992) proposed that recollecting the days with binge eating episodes is performed more accurately than recollecting the number of binge eating episodes, we measured the number of days in which an objective binge episode had occurred during the last 28-days.

Until now, most studies with obese persons only focused on weight loss or binge episodes as outcome measures. It is valuable to use a broader outcome spectrum which includes cognitions regarding shape, weight and eating. In order to enable more meaningful comparisons among the literature it would be useful to use standard, well validated assessment instruments, such as the Eating Disorder Examination self-report version or the investigator-based interview (Wilfley et al., 2000). Finally, a core set of internationally used instruments to measure depression and self-esteem would allow an optimum pooling of a broad outcome spectrum for obesity.

\section{USE OF PROTOCOLS IN CLINICAL PRACTICE}

Despite the level of interest in developing and validating protocols, it is difficult to implement empirically supported therapies in clinical practice (Wilson, 1996a; 1997). Empirically supported therapies have been accused of using inflexible manuals, excluding 'real' patients, and undermining therapists clinical artistry (Wilson, 1996a; 1997). Wilson (1996a; 1997) mentioned a number of arguments against these criticisms. First, when using protocols in clinical practice greater flexibility is possible than often realized by therapists. If one technique is not working, another empirically-validated technique can be tried. Furthermore, treatment manuals vary greatly in degree of structure and standardization, and manuals vary also in the way individualization is allowed. Second, randomized controlled trials commonly include patients with multiple 
problems and levels of disturbance as severe as patients in clinical settings. Third, the importance of developing rapport and building a positive therapeutic alliance is no less important than in traditional therapy.

It is important to investigate how best to implement empirical based manualized treatment programs on a wider scale in clinical practice. Some researchers have suggested that treatment innovation is a developmental process including four subsequent stages: dissemination, adoption, implementation, and continuation (e.g., Paulussen, 1994; Rogers, 1983; Schaalma, 1995). At the dissemination stage potential users are informed about the innovation. In the adoption stage potential users have the intention to use the innovation. In the implementation stage the innovation is actual used and ideally continuation succeeds initial implementation. To improve the actual implementation of protocols in clinical practice several strategies could be used such as: interpersonal communication, writing in scientific and popular magazines about the protocols, presentations on conferences, and organizing workshops in which therapists are trained to deliver the protocols.

\section{RECOMMENDATIONS FOR CLINICAL PRACTICE}

In this thesis it was shown that at short and long-term follow-up the cognitive and behavioral therapy were both effective. However, the cognitive therapy was more effective in reducing a broad spectrum of complaints than the behavioral therapy. The protocols of the interventions may serve as a tool for specialized therapist in (mental) health settings to improve psychological well being, dysfunctional cognitions, and eating patterns of obese persons with and without BED.

There has been a recent trend to provide cognitive-behavioral therapy via self-administered or therapist-administered self-help programs. Results indicated that these less intensive self-help programs were effective in reducing eating pathology and improving self-esteem for some obese binge eaters (Carter \& Fairburn, 1998; Garvin, Striegel-Moore, \& Wells, 1998; Loeb, Wilson, Gilbert, \& Labouvie, 2000; Peterson et al., 1998; Wells, Garvin, Dohm, \& StriegelMoore, 1997) and obese non-binge eaters (Meyers, Graves, Whelan, \& Barclay, 1996). These findings have several implications for a stepped-care strategy in treating obesity and BED. The philosophy behind a stepped-care strategy is that the easiest and cheapest approaches would be undertaken as the first line of intervention. Treatment drop-outs will receive more intensive treatment approaches (Wilson, Vitousek, \& Loeb, 2000). It can be argued that for a large group of obese non-binge eaters and a smaller group of obese binge eaters a self-help manual is sufficient.

Participants who are poor responders to self-help manuals need a cognitive 
or behavioral therapy given by specialized therapists as mentioned in this thesis. Finally, Agras and colleagues (1995) have suggested that the highest level intervention was needed for participants who were also poor responders to cognitive-behavioral therapy. They evaluated the effectiveness of 12 weeks IPT for cognitive-behavioral non-responders and found that IPT did not result in further improvement for cognitive-behavioral non-responders. In a study of Eldredge et al. (1997), where an extended CBT was administered to non-responders, it was found that $43 \%$ of the initial non-responders no longer met the BED criteria at the end of treatment. It might also be necessary for a subgroup of particularly BED patients to treat the comorbid axis-II disorder. Prevalence rates for borderline personality disorder among persons with BED in eating disorders and mental health setting varied from $6 \%$ to $30 \%$ (Sanone et al., 2000). It is a challenge for future research to optimize treatment outcome for non-responders. Furthermore, researchers need to understand which type of treatment is best suited for which obese persons. Therefore, an important clinical issue is to find out which pretreatment characteristics of obese persons do predict outcome. Finding reliable predictors of treatment outcome is still in its infancy and reliable predictors of treatment outcome have yet to be established (Wilson, 1994).

Given the lack of effective treatments among obese adults to lose and maintain weight loss, prevention of obesity is all the more a priority (Devlin, Yanovski, \& Wilson, 2000). A notable contrast to the high relapse rates among obese adults is the outcome in children. Research has shown that weight loss could be maintained over a 10-year follow-up in children, even though their parents showed predictable relapse rates (Epstein, Valosky, Wing, \& McCurley, 1994). It will be important to encourage healthy eating and exercise habits, and the development of healthy shape and weight related cognitions at an early age.

The present thesis has shown that shape and weight concerns do play a central role in the maintenance of obesity and cognitive and behavioral therapy is effective in lowering these concerns. Negative attitudes towards obese persons are widespread in Western countries and obese persons are one of the stigmatized groups in our society (Crandall, 1994). To reduce shape and weight concerns in obese persons, it is also important to tackle negative social reactions towards obese persons. The famous Venus of Willendorf, a small 25.000-yearold statue of a very heavy female figure, was viewed in a positive way. Maybe, it is now time to change our reactions towards obese persons. 


\section{REFERENCES}

Adami, G.F., Gandolfo, P., Bauer, B., \& Scopinaro, N. (1995). Binge eating in massively obese patients undergoing bariatric surgery. International Journal of Eating Disorders, 17, 45-50.

Adami, G.F., Gandolfo, P., Campostano, A., Bauer, B., Cocchi, F., \& Scopinaro, N. (1994). Eating disorder inventory in the assessment of psychosocial status in the obese patients prior to and at long-term following bibliopancreatic diversion for obesity. International journal of Eating Disorders, 15, 265-274.

Adami, G.F., Gandolfo, P., Campostano, A., Meneghelli, A., Ravera, G., \& Scopinaro, N. (1998). Body image and body weight in obese persons. International Journal of Eating Disorders, 24, 299-306.

Agras, W.S., Schneider, J.A., Arnow, B., Raeburn, S.D., \& Telch, C.F. (1989). Cognitive-behavioral and response-prevention treatment for bulimia nervosa. Journal of Consulting and Clinical Psychology, 57, 215-221.

Agras, W.S., \& Telch, C.F. (1998). The effects of caloric deprivation and negative affect on binge eating in obese binge-eating disordered women. Behavior Therapy, 29, 491-503.

Agras, W.S., Telch, C.F.,Arnow, B., Eldredge, K., Henderson, J., \& Marnell, M. (1995). Does interpersonal therapy help patients with binge eating disorder who fail to respond to cognitive-behavioral therapy? Journal of Consulting and Clinical Psychology, 63, 356-360.

Agras, W.S., Telch, C.F., Arnow, B., Eldredge, K., \& Marnell, M. (1997). One-year follow-up of cognitive-behavioral therapy for obese individuals with binge eating disorder. Journal of Consulting and Clinical Psychology, 65, 343-347.

Agras, W.S., Telch, C.F., Arnow, B., Eldredge, K., Wilfley, D.E., Raeburn, S.D., Henderson, J., \& Marnell, M. (1994). Weight loss, cognitive-behavioral, and desipramine treatments in binge eating disorder:An additive design. Behovior Therapy, 25, 225-238.

Allen, H.N., \& Craighead, L.W. (1999). Appetite monitoring in the treatment of binge eating disorder. Behavior Therapy, 30, 253-272.

American Psychiatric Association (1994). Diagnostic and statistical manual of mental disorders (4th ed.). Washington, DC:Author.

Arnow, B., Kenardy, J., \& Agras, W.S. (1992). Binge eating among the obese:A descriptive study. Journal of Behavioral Medicine, 15, 155-170.

Beck,A.T. (1967). Depression: Clinical, experimental, and theoretical aspects. New York: Hoeber.

Beck, A.T. (1976). Cognitive therapy and the emotional disorders. New York: International Universities Press.

Beck, A.T. (1996). Beyond belief:A theory of modes, personality, and psychopathology. In P.M. Salkovskis (Ed.), Frontiers of cognitive therapy (pp. 1-25). New York: Guilford Press.

Beck, A.T., Steer, R.A., \& Garbin, M.G. (1988). Psychometric properties of the Beck Depression Inventory:Twenty-five years of evaluation. Clinical Psychology Review, 8, 77-100.

Beck, A.T., Ward, C.H., Mendelson, M., Mock, J., \& Erbaugh, J. (1961). An inventory for measuring depression. Archives of General Psychiatry, 4, 561-571.

Beck,A.T.,Wright, F.D., Newman, C.F., \& Liese, B.S. (1993). Cognitive therapy of substance abuse. New York: Guilford Press.

Bennett, G.A. (1986). Behavior therapy for obesity: A quantitative review of the effects of selected treatment characteristics on outcome. Behovior Therapy, 17, 554-562. 
Blackburn, G.L., Wilson, G.T., Kanders, B.S., Stein, L.J., Lavin, P.T., Adler, J., \& Brownell, K.D. (1989). Weight cycling: The experience of human dieters. American Journal of Clinical Nutrition, 49, $1105-1109$.

Blair, S.N., Shaten, J., Brownell, K., Collins, G., \& Lissner, L. (1993). Body weight change, allcause mortality, and cause-specific mortality in the Multiple Risk Factor Intervention Trial. Annals of Internal Medicine, I/9, 749-757.

Bouchard, C., \& Perusse, L. (1993). Genetics of obesity. Annual Review of Nutrition, 13, 337. 354.

Bouchard, C., Perusse, L., Leblanc, C., Tremblay, A., \& Theriault, G. (1988). Inheritance of the amount and distribution of human body fat. International Journal of Obesity, 12, 205-215.

Bray, G.A. (1990). Obesity: Historical development of scientific and cultural ideas. International Journal of Obesity, 14, 909-926.

Brody, M.L., Walsh, B.T., \& Devlin, M.J. (1994). Binge eating disorder: Reliability and validity of a new diagnostic category. Journal of Consulting and Clinical Psychology, 62, 381-386.

Brown, P.J., \& Bentley-Condit, V.K. (1998). Culture, evolution and obesity. In G.A. Bray, C. Bouchard, \& W.P.T. James (Eds.), Handbook of obesity (pp. 143-155). New York: Marcel Dekker Inc.

Brownell, K.D. (1992). Relapse and the treatment of obesity. In T.A. Wadden, \& T.B.Van Itallie (Eds.), Treatment of the seriously obese patient (pp. 437-455). London: Guilford Press.

Brownell, K.D. (1995). History of obesity. In K.D. Brownell, \& C.G. Fairburn (Eds.), Eating Disorders and Obesity:A comprehensive handbook (pp. 386-391). New York: Guilford Press.

Brownell, K.D., Greenwood, M.R.C., Stellar, E., \& Shrager, E.E. (1986). The effects of repeated cycles of weight loss and regain in rats. Physiology and Behavior, 38, 459-464.

Brownell, K.D., \& Rodin, J. (1994). The dieting maelstrom: Is it possible and advisable to lose weight? American Psychologist, 49, 781-791.

Brownell, K.D., \& Wadden, T.A. (199I). The heterogeneity of obesity: Fitting treatments to individuals. Behovior Therapy, 22, 153-177.

Brownell, K.D., \& Wadden, T.A. (1992). Etiology and treatment of obesity: Understanding a serious, prevalent, and refractory disorder. Journal of Consulting and Clinical Psychology, 60, 505-517.

Bruce, B., \& Agras, W.S. (1992). Binge eating in females: A population based investigation. International Journal of Eating Disorders, 12, 365-373.

Bruce, B., \& Wilfley, D. (1996). Binge eating among the overweight population:A serious and prevalent problem. Journal of the American Dietetic Association, 96, 58-61.

Bulik, C.M., Sullivan, P.F., Joyce, P.R., Carter, F.A., \& McIntosh, V.V. (1998). Predictors of I-year outcome in bulimia nervosa. Comprehensive Psychiatry, 39, 206-214.

Carter, J.C., \& Fairburn, C.G. (1998). Cognitive-behavioral self-help for binge eating disorder: A controlled effectiveness study. Journal of Consulting and Clinical Psychology, 66, 616-623.

Cash, T.F., Counts, B., \& Huffine, C.E. (1990). Current and vestigal effects of overweight among women: Fear of fat, attitudinal body image, and eating behaviors. Journal of Psychopathology and Behavioral Assessment, 12, 157-167.

Castonguay, L.G., Eldredge, K.L., \& Agras, W.S. (1995). Binge eating disorder: Current state and future directions. Clinical Psychology Review, 15, 865-890.

Centraal Bureau voor Statistiek (200I). Gezondheidstoestand van de Nederlands bevolking. Statline CBS: http://statline.cbs.nl/statweb/index.stm. 
Ciliska, D. (1990). Beyond dieting - Psychoeducational interventions for chronically obese women: A non-dieting approach. New York: Brunner/Mazel.

Ciliska, D. (1998). Evaluation of two nondieting interventions for obese women. Western Journal of Nursing Research, 20, 119-135.

Clark, D.A. (1988). The validity of measures of cognition: A review of the literature. Cognitive Therapy and Research, 12, 1-20.

Clark, D.A., \& Steer, R.A. (1996). Empirical status of the cognitive model. In P.M. Salkovskis (Ed.), Frontiers of cognitive therapy (pp. 75-96). New York: Guilford Press.

Collins, J.K., Beumont, P.J.V., Touyz, S.W., Krass, J., Thompson, P., \& Philips, T. (1987).Variability in body shape perception in anorexic, bulimic, obese and control subjects. International Journal of Eating Disorders, 6, 633-638.

Collins, R.L., Rothblum, E.D., \& Wilson, G.T. (1986). The comparative efficacy of cognitive and behavioral approaches to the treatment of obesity. Cognitive Therapy and Research, 10, 299. 318.

Cooper, M. (1997). Cognitive theory in anorexia nervosa and bulimia nervosa: A Review. Behavioural and Cognitive Psychotherapy, 25, 113-145.

Cooper, M. Cohen-Tovée, E., Todd, G.Wells, A., \& Tovée, M. (1997). The eating disorder belief questionnaire: Preliminary development. Behaviour Research and Therapy, 4, 381-388.

Cooper, M.J., \& Fairburn, C.G. (1992). Thoughts about eating, weight and shape in anorexia nervosa and bulimia nervosa. Behaviour Research and Therapy, 30, 501-51I.

Craighead, L.W., \& Allen, H.N. (1995). Appetite awareness training: A cognitive-behavioral intervention for binge eating. Cognitive and Behavioral Practice, 2, 249-270.

Crandall, C.S. (1994). Prejudice against fat people: Ideology and self-interest. Journal of Personality and Social Psychology, 66, 882-894.

Davis, R., Freeman, R. J., \& Garner, D. M. (1988). A naturalistic investigation of eating behavior in bulimia nervosa. Journal of Consulting and Clinical Psychology, 56, 273-279.

De Haan, E., van Oppen, P., van Balkom, A.J.L.M., Spinhoven, P., Hoogduin, C.A.L., \& van Dyck, R. (1997). Prediction of outcome and early vs. late improvement in OCD patients treated with cognitive behaviour therapy and pharmacotherapy. Acta Psychiatrica Scandinavio, 96, 354-361.

Devlin, M.J., Yanovski, S.Z., \& Wilson, G.T. (2000). Obesity: What mental health professionals need to know. The American Journal of Psychiatry, 157, 854-866.

De Zwaan, M., Bach, M., Mitchell, J.E.,Ackard, D., Specker, S.M., Pyle, R.L., \& Pakesch, G. (1995). Alexithymia, obesity, and binge eating disorder. International Journal of Eating Disorders, I7, 135-140.

De Zwaan, M., Mitchell, J.E., Seim, H.C., Specker, S.M., Pyle, R.L., Raymond, N.C., \& Crosby, R.B. (1994). Eating related and general psychopathology in obese females with binge eating disorder. International Journal of Eating Disorders, 15, 43-52.

De Zwaan, M., Nutzinger, D.O., \& Schoenbeck, G. (1992). Binge eating in overweight women. Comprehensive Psychiatry, 33, 256-26I.

DiClemente C.C., \& Prochaska, J.O. (1998). Toward a comprehensive transtheoretical model of change: Stages of change and addictive behaviors. In W.R. Miller, \& N. Heather (Eds.), Treating addictive behaviors (pp. 3-24). New York: Plenum Press.

Dritschel, B.H., Williams, K., \& Cooper, P.J. (1991). Cognitive distortions amongst women experiencing bulimic episodes. International Journal of Eating Disorders, 10, 547-555. 
Eldredge, K.L., \& Agras, W.S. (1996). Weight and shape overconcern and emotional eating in binge eating disorder. International journal of Eating Disorders, 19, 73-82.

Eldredge, K.L., Agras, W.S., Arnow, B., Telch, C.F., Bell, S., Castonguay, L., \& Marnell, M. (1997). The effects of extending cognitive-behavioral therapy for binge eating disorder among initial treatment nonresponders. International Journal of Eating Disorders, 21, 347-352.

Epstein, L.H., Valoski, A., Wing, R.R., \& McCurley, J. (1994). Ten-year outcomes of behavioral family-based treatment for childhood obesity: Theoretical and practical implications. Health Psychology, 13, 373-383.

Fairburn, C.G. (1985). Cognitive-behavioral treatment for bulimia. In D.M. Garner, \& P.E. Garfinkel (Eds.), Handbook of Psychotherapy for Anorexia and Bulimia (pp. 160-192). New York: Guilford Press.

Fairburn, C.G. (1987). The definition of bulimia nervosa: Guidelines for clinicians and research workers. Annals of Behavioral Medicine, 9, 3-7.

Fairburn, C.G., \& Beglin, S.J. (1994). Assessment of eating disorders. Interview or self-report questionnaire? International Journal of Eating Disorders, 16, 363-370.

Fairburn, C.G., \& Cooper, Z. (1993). The Eating Disorder Examination (12th ed.). In C.G. Fairburn, \& G.T.Wilson (Eds.), Binge eating: Nature, assessment, and treatment (pp. 317-360). New York: Guilford Press.

Fairburn, C.G., Marcus, M.D., \& Wilson, G.T. (1993). Cognitive-behavioral therapy for binge eating and bulimia nervosa: A comprehensive treatment manual. In C.G. Fairburn, \& G.T. Wilson (Eds.), Binge eating: Nature, assessment, and treatment (pp. 36I-404). New York: Guilford Press.

Fairburn, C.G., Peveler, R.C., Jones, R., Hope, R.A., \& Doll, H.A. (1993). Predictors of I2month outcome in bulimia nervosa and the influence of attitudes to shape and weight. Journal of Consulting and Clinical Psychology, 61, 696-698.

Ferster, C.B., Nurnberger, J.I., \& Levitt, E.E. (1962). The control of eating. Journal of Mathetics, I,87-109.

Fichter, M.M., Quadflieg, N., \& BrandI, B. (1993). Recurrent overeating: An empirical comparison of binge eating disorder, bulimia nervosa, and obesity. International Journal of Eating Disorders, 14, 1-16.

122 Foreyt, J.P., Brunner, R.L., Goodrick, G.K., Cutter, G., Brownell, K.D., \& Jeor, S.T. (1995). Psychological correlates of weight fluctuation. International Journal of Eating Disorders, I7, 263-275.

Foster, G.D., Sarwer, D.B., \& Wadden, T.A. (1997). Psychological effects of weight cycling in obese persons:A review and research agenda. Obesity Research, 5, 474-487.

Foster, G.D., \& Wadden, T.A. (1994). The psychology of obesity, weight loss and weight regain: Research and clinical findings. In G.L. Blackburn, \& B.S. Kanders (Eds.), Obesity: Pathophysiology, psychology and treatment (pp. 140-166). New York: Chapman and Hall.

Friedman, M.A., \& Brownell, K.D. (1995). Psychological correlates of obesity: Moving to the next research generation. Psychological Bulletin, II7, 3-20.

Gale, E.N., \& Funck, P.D. (1984). Factors associated with successful outcome from behavioral therapy for chronic temporomandibular joint pain. Journal of Psychosomatic Research, 28, 441-448.

Garfield, S.L. (1994). Research on client variables in psychotherapy. In A.E. Bergin, \& S.L. Garfield (Eds.), Handbook of psychotherapy and behavior change (pp. 190-228). New York: Wiley \& Sons. 
Garner, D.M., \& Bemis, K.M. (1982). A cognitive-behavioral approach to anorexia nervosa. Cognitive Therapy and Research, 6, 123-150.

Garner, D.M., Olmsted, M.P., \& Polivy, J. (1983). Development and validation of a multidimensional eating disorder inventory for anorexia nervosa and bulimia. International journal of Eating Disorders, 2, 15-34.

Garner, D.M., \& Wooley, S.C. (1991). Confronting the failure of behavioral and dietary treatments for obesity. Clinical Psychology Review, II, 729-780.

Garvin, V., Striegel-Moore, R.H., \& Wells, A.M. (1998). Participant reactions to a cognitivebehavioral guided self-help program for binge eating: Developing criteria for program evaluation. Journal of Psychosomatic Research, 44, 407-412.

Gladis, M.M., Wadden, T.A., Vogt, R., Foster, G., Kuehnel, R.H., \& Bartlett, S.J. (1998). Behavioral treatment of obese binge eaters: Do they need different care? Journal of Psychosomatic Research, 44, 375-384.

Goodrick, G.K., Poston, W.S., Kimball, K.T., Reeves, R.S., \& Foreyt, J.P. (1998). Nondieting versus dieting treatment for overweight binge-eating women. Journal of Consulting and Clinical Psychology, 66, 363-368.

Gormally, J., Black, S., Daston, S., \& Rardin, D. (1982). The assessment of binge eating severity among obese persons. Addictive Behaviors, 7, 47-55.

Gotestam, K.G., \& Agras, W.S. (1995). General population-based epidemiological study of eating disorders in Norway. International Journal of Eating Disorders, 18, 119-126.

Greeno, C.G.,Wing, R.R., \& Marcus, M.D. (1999). How many donuts is a "binge"? Women with BED eat more but do not have more restrictive standards than weight-matched non-BED women. Addictive Behaviors, 24, 299-303.

Greeno, C.G.,Wing, R.R., \& Shiffman, S. (2000). Binge antecedents in obese women with and without binge eating disorder. Journal of Consulting and Clinical Psychology, 68, 95-102.

Grilo, C.M., Wilfley, D.E., Jones, A., Brownell, K.D., \& Rodin, J. (1994). The social self, body dissatisfaction, and binge eating. Obesity Research, 2, 24-27.

Guydish, J., \& Greenfield, T.K. (1990). Alcohol related cognitions. Do they predict treatment outcome? Addictive Behaviors, 15, 423-430.

Hamm, P., Shekelle, R.B., \& Stamler, J. (1989). Large fluctuations in body weight during young adulthood and twenty-five-year risk of coronary death in men. American Journal of Epidemiology, 129, 312-318.

Harris, M.B., Walters, L.C., \& Waschull, S. (1991). Gender and ethnic differences in obesity related behaviors and attitudes in a college sample. The Journal of Social Psychology, 21, 1545-1566.

Heitmann, B.L., \& Lissner, L. (1995). Dietary underreporting by obese individuals - Is it specific or non-specific? British Medical Journal, 311, 986-989.

Herman, C.P., \& Mack, D. (1975). Restrained and unrestrained eating. Journal of Personality, 43, 647-660.

Herman, C.P., \& Polivy, J. (1980). Restrained eating. In A.J. Stunkard (Ed.), Obesity. Philadelphia: W.B. Saunders.

Herman, C.P., \& Polivy, J. (1983). A Boundary Model for the regulation of eating. Psychiatric Annals, 13, 918-927.

Hibscher, J.A., \& Herman, C.P. (1977). Obesity, dieting, and the expression of obese characteristics. Journal of Comparative and Physiological Psychology, 91, 374-380. 
Higgins, M., D'Agostino, R., Kannel, W., \& Cobb, J. (1993). Benefits and adverse effects of weight loss: Observations from the Framingham study. Annals of Internal Medicine, I/9, 758-763.

Horm, J., \& Anderson, K. (1993). Who in America is trying to lose weight? Annals of Internal Medicine, II9, 672-676.

Howard, C.E., \& Porzelius, L.K. (1999). The role of dieting in binge eating: Etiology and treatment implications. Clinical Psychology Review, 19, 25-44.

Hudson, J.I., Pope, H.G., Wurtman, J., Yurgelun-Todd, D., Mark, S., \& Rosenthal, N.E. (1988). Bulimia in obese individuals: Relationship to normal-weight bulimia. The Journal of Nervous and Mental Disease, 176, 144-152.

Hunt, D.A., \& Rosen, J.C. (1981). Thoughts about food by obese and nonobese individuals. Cognitive Therapy and Research, 5, 317-322.

Institute of Medicine (1995). Weighing the options: Criteria for evaluating weight management programs. Washington, DC: National Academy Press.

Jansen, A. (1998). Eating Disorders. In A.S. Bellack, \& M. Hersen (Eds.), Comprehensive Clinical Psychology (pp. 649-667). Oxford: Elsevier Science Limited, Pergamon Press.

Jeffery, R.W., Drewnowski,A., Epstein, L.H., Stunkard,A.J., Wilson, G.T., Wing, R.R., \& Hill, D.R. (2000). Long-term maintenance of weight loss: Current status. Health Psychology, 19, 5-16.

Johnson, W.G., Tsoh, J.Y., \& Varnado, P.J. (1996). Eating disorders: Efficacy of pharmacological and psychological disorders. Clinical Psychology Review, 16, 457-478.

Keefe, P.H.,Wyshogrod, D., Weinberger, E., \& Agras, W.S. (1984). Binge eating and outcome of behavioral treatment of obesity: A preliminary report. Behaviour Research and Therapy, 22, 319-321.

Keijsers, G.P.J., Hoogduin, C.A.L., \& Schaap, C.P.D.R. (1994). Prognostic factors in the behavioral treatment of panic disorder with and without agoraphobia. Behavior Therapy, 25, 689708.

Keys, A., Brozek, J., Henschel, A., Mickelsen, O., \& Taylor, H.L. (1950). The biology of human starvation. Minneapolis: University of Minnesota.

Kluthe, R., \& Schubert, A. (1985). Obesity in Europe. Annals of Internal Medicine, 103, 1037. 1042.

Kristenson, H. (1992). Long-term antabuse treatment of alcohol dependent patients. Acto Psychiatrica Scandinavia, 86, 41-45.

Kuczmarski, R.J., Flegal, K.M., Campbell, S.M., \& Johnson, C.L. (1994). Increasing prevalence of overweight among US adults. The National Health and Nutrition Examination Surveys, 1960 to 1991. Journal of the American Medical Association, 272, 205-21 I.

Kuehnel, R.H., \& Wadden, T.A. (1994). Binge eating disorder, weight cycling, and psychopathology. International Journal of Eating Disorders, 15, 321-329.

Lambert, M.J., Shapiro, D.A., \& Bergin, A.E. (1986). The effectiveness of psychotherapy. In S.L. Garfield, \& A.E. Bergin (Eds.), Handbook of psychotherapy and behavior change (pp. 157-213). New York: John Wiley \& Sons.

Leary, M.R., Schreindorfer, L.S., \& Haupt,A.L. (1995). The role of low self-esteem in emotional and behavioral problems:Why is low self-esteem dysfunctional? Journal of Social and Clinical Psychology, 14, 297-314.

Lee, I.M., \& Paffenbarger, R.S. (1992). Changes in body weight and longevity. Journal of the American Medical Association, 268, 2045-2049. 
Lewis, C.E., Jacobs, D.R., McCreath, H., Kiefe, C.I., Schreiner, P.J., Smith, D.E., \& Williams, O.D. (2000). Weight gain continues in the 1990s: 10-year trends in weight and overweight from the CARDIA study. American Journal of Epidemiology, 151, 1172-1181.

Lissner, L., Odell, P.M., D’Agostino, R.B., Stokes, J., Kreger, B.E., Belanger, A.J., \& Brownell, K.D. (1991). Variability in body weight and health outcomes in the Framingham population. New England Journal of Medicine, 324, 1839-1844.

Lloyd-Richardson, E.E., King, T.K., Forseyth, L.H., Clark, M.M. (200). Body image evaluations in obese females with binge eating disorder. Eating Behaviors, I, |61-171.

Loeb, K.L.,Wilson, G.T., Gilbert, J.S., \& Labouvie, E. (2000). Guided and unguided self-help for binge eating. Behaviour Research and Therapy, 38, 259-272.

Long, C.G., Hinton, C., \& Gillespie, N.K. (1994). Selective processing of food and body size words: Application of the Stroop test with obese restrained eaters, anorexics, and normals. International Journal of Eating Disorders, 15, 279-283.

Lowe, M.R. (1993). The effects of dieting on binge eating behavior: A three-factor model. Psychological Bulletin, I/4, 100-121.

Luce, K.H., \& Crowther, J.H. (1999). The reliability of the Eating Disorder Examination - selfreport questionnaire version (EDE-Q). International Journal of Eating Disorders, 25, 349-35I.

Mahoney, M.J., \& Mahoney, K. (1976). Permanent weight control:A total solution to the dieters dilemma. New York: Norton.

Malkoff, S.B., Marcus, M.D., Grant, A., Moulton, M.M., \& Vayonis, C. (1993). The relationship between dieting and binge eating among obese individuals. Annals of Behovioral Medicine, 15, S40.

Manore, M.M., Berry, T.E., Skinner, J.S., \& Caroll, S.S. (1991). Energy expenditure at rest and during exercise in nonobese female cyclical dieters and in nondieting control subjects. American Journal of Clinical Nutrition, 54, 41-46.

Marcus, M.D., Smith, D., Santelli, R., \& Kaye, W. (1992). Characterization of eating disordered behavior in obese binge eaters. International Journal of Eating Disorders, 12, 249-255.

Marcus, M.D., Wing, R.R., Ewing, L., Kern, E., Gooding,W., \& McDermott, M. (1990). Psychiatric disorders among obese binge eaters. International Journal of Eating Disorders, 9, 69-77.

Marcus, M.D.,Wing, R.R., \& Fairburn, C.G. (1995). Cognitive treatment of binge eating versus behavioral weight control in the treatment of binge eating disorder. Annals of Behavioral Medicine, 17, S090.

Marcus, M.D., Wing, R.R., \& Hopkins, J. (1988). Obese binge eaters: Affect, cognitions, and response to behavioral weight control. Journal of Consulting and Clinical Psychology, 56, 433439.

Marcus, M.D., Wing, R.R., \& Lamparski, D.M. (1985). Binge eating and dietary restraint in obese patients. Addictive Behoviors, 10, 163-168.

Markus, H. (1977). Self-schemata and processing information about the self. Journal of Personality and Social Psychology, 35, 63-78.

Markus, H., Hamill, R., \& Sentis, K.P. (1987). Thinking fat: Self-schemas for body weight and the processing of weight relevant information. Journal of Applied Social Psychology, 17, 50-7I.

Markus, H., \& Sentis, K. (1982). The self in social information processing. In J. Suls (Ed.), Psychological perspectives on the self. New York: Lawrence Erlbaum.

Marlatt, G.A., \& Gordon, J.R. (1985). Relapse Prevention: Maintenance strategies in the treatment of addictive behaviors. New York: Guilford Press. 
Masheb, R.M., \& Grilo, C.M. (2000). Binge eating disorder: A need for additional diagnostic criteria. Comprehensive Psychiatry, 4I, 159-162.

Mathews, A.M., \& MacLeod, C. (1985). Selective processing of treat cues in anxiety states. Behoviour Research and Therapy, 23, 563-569.

Meyers, A.W., Graves, T.J., Whelan, J.P., \& Barclay, D.R. (1996). An evaluation of a televisiondelivered behavioral weight loss program: Are the ratings acceptable? Journal of Consulting and Clinical Psychology, 64, $172-178$.

Miller, C.T., \& Downey, K.T. (1999).A meta-analysis of heavyweight and self-esteem. Personality and Social Psychology Review, 3, 68-84.

Mitchell, J.E., \& Mussell, M.P. (1995). Comorbidity and binge eating disorder. Addictive Behaviors, 20, 725-732.

Mussell, M.P., Mitchell, J.E., DeZwaan, M. Crosby, R.D., Seim, H.C., \& Crow, S.J. (1996). Clinical characteristics associated with binge eating in obese females: A descriptive study. International Journal of Obesity, 20, 324-331.

Mussell, M.P., Mitchell, J.E., Weller, C.L., Raymond, N.C., Crow, S.J., \& Crosby, R.D. (1995). Onset of binge eating, dieting, obesity, and mood disorders among subjects seeking treatment for binge eating disorder. International Journal of Eating Disorders, 17, 395-40I.

Mussell, M.P., Peterson, C.B., Weller, C.L., Crosby, R.D., de Zwaan, M., \& Mitchell, J.E. (1996). Differences in body image and depression among obese women with and without binge eating disorder. Obesity Research, 4, 431-439.

National Task Force on the Prevention and Treatment of Obesity (1994). Weight cycling. Journal of the American Medical Association, 272, 1196-1202.

Nauta, H. (200I). Protocollaire groepsbehandeling voor obese vrouwen met en zonder eetbuistoornis: Gedragstherapie en cognitieve therapie. Gedrag en Gezondheid, 29, 77-85.

Nauta, H., Hospers, H.J., Jansen, A., \& Kok, G. (2000). Cognitions in obese binge eaters and obese non-binge eaters. Cognitive Therapy and Research, 24, 521-531.

Nauta, H., Hospers, H., Kok, G., \& Jansen, A. (2000). A comparison between a cognitive and a behavioral treatment for obese binge eaters and obese non-binge eaters. Behavior Therapy, $31,441-46 I$.

Nauta, H., Hospers, H., \& Jansen, A. (200I). One-year follow-up effects of two obesity treatments on psychological well-being and weight. British Journal of Health Psychology, 6 (3), $271-284$.

NHLBI Obesity Education Initiative Expert Panel (1998). Clinical guidelines of the identification, evaluation, and treatment of overweight and obesity in adults. The Evidence Report. Obesity Research, 6, 5IS-2IOS.

Nisbett, R.E. (1972). Hunger, obesity and the ventromedial hypothalamus. Psychological Review, 79, 433-453.

O'Connor, J., \& Dowrick, P.W. (1987). Cognitions in normal weight, overweight, and previously overweight adults. Cognitive Therapy and Research, II, 315-326.

Omer, H., \& London, P. (1989). Signal and noise in psychotherapy: The role and control of non-specific factors. British Journal of Psychiatry, I55, 239-245.

Pamuk, E.R. Williamson, D.F., Serdula, M.K., Madans, J., \& Byers, T.E. (1993). Weight loss and subsequent death in a cohort of US adults. Annals of Internal Medicine, I I9, 744-748.

Paulussen, T.G.W. (1994). Adoption and implementation of AIDS education in Dutch secondary schools. Utrecht, The Netherlands: National Center for Health Promotion and Health Education. 
Perri, M.G., McAdoo, W.G., McAllister, D.A., Lauer, J.B., Jordan, R.C., \& Nezu, A.M. (1987). Effects of peer support and therapist contact on long-term weight loss. Journal of Consulting and Clinical Psychology, 55, 615-617.

Perri, M.G., McAllister, D.A., Gange, J.J., Jordan, R.C., McAdoo, W.G., \& Nezu, A.M. (1988), Effects of four maintenance programs on the long-term management of obesity. Journal of Consulting and Clinical Psychology, 56, 529-534.

Perri, M.G., Nezu, A.M., Patti, E.P., \& McCann, K.L. (1989). Effect of length of treatment on weight loss. Journal of Consulting and Clinical Psychology, 57, 450-452.

Peterson, C.B., Mitchell, J.E., Engbloom, S., Nugent, S., Mussell, M.P., Miller, J.P. (1998), Group cognitive-behavioral treatment of binge eating disorder: A comparison of therapist-led versus self-help formats. International Journal of Eating Disorders, 24, 125-136.

Phelan, P.W. (1987). Cognitive correlates of bulimia: The Bulimic Thoughts Questionnaire. International Journal of Eating Disorders, 6, 593-607.

Pingitore, R., Spring, B., Garfield, D. (1997). Gender differences in body satisfaction. Obesity Research, 5, 402-409.

Polivy, J., \& Herman, C.P. (1983). Breaking the diet habit:The natural weight alternative. New York: Basic Books.

Polivy, J., \& Herman, C.P. (1985). Dieting and bingeing: A causal analysis. American Psychologist, 40, 193-201.

Polivy, J., \& Herman, C.P. (1992). Undieting: A program to help people stop dieting. International Journal of Eating Disorders, II, 26I-268.

Polivy, J., \& Herman, C.P. (1995). Dieting and its relation to eating disorders. In K.D. Brownell, \& C.G. Fairburn (Eds.), Eating Disorders and Obesity: A comprehensive handbook (pp. 83-92). New York: Guilford Press.

Porzelius, L.K., Houston, C., Smith, M., Arfken, C., \& Fisher, E. (1995). Comparison of a standard behavioral weight loss treatment and a binge eating weight loss treatment. Behavior Therapy, 26, I19-134.

Prochaska, J.O., \& DiClemente, C.C. (1983). Stages and processes of self-change of smoking: Toward an integrative model of change. Journal of Consulting and Clinical Psychology, 51,390 395.

Prochaska, J.O., Norcross, J.C., Fowler, J.L., Follick, M.J., \& Abrams, D.B. (1992).Attendance and outcome in a worksite weight control program: Processes and stages of change as process and predictor variables. Addictive Behaviors, 17, 35-45.

Reed, G.W., \& Hill, J.O. (1993). Weight cycling: A review of the animal literature. Obesity Research, I, 392-402.

Rodin, J. (198I). Current status of the internal-external hypothesis for obesity: What went wrong? American Psychologist, 36, 361-372.

Rodin, J., Radke-Sharpe, N., Rebuffé-Scrive, M., \& Greenwood, M.R. (1990). Weight cycling and fat distribution. International Journal of Obesity, 14, 303-310.

Rogers, E.M. (1983). Diffusion of innovations. New York:The Free Press.

Rosen, J.C., Orosan, P., \& Reiter, J. (1995). Cognitive behavior therapy for negative body image in obese women. Behavior Therapy, 26, 25-42.

Rosenberg, M. (1965). Society and the adolescent self-image. Princeton, NJ: Princeton University Press. 
Rossiter, E.M., Agras, W.S., Telch, C.F., \& Bruce, B. (1992). The eating patterns of non-purging bulimic subjects. International Journal of Eating Disorders, II, II I- 120.

Ruderman, A.J. (1986). Dietary restraint: A theoretical and empirical review. Psychological Bulletin, 99, 247-262.

Sansone, R.A., Wiederman, M.W., \& Sansone, L.A. (2000). The prevalence of borderline personality disorder among individuals with obesity: A critical review of the literature. Eating Behaviors, I, 93-104.

Sarwer, D.B., Wadden, T.A., \& Foster, G.D. (1998). Assessment of body image dissatisfaction in obese women: Specificity, severity, and clinical significance. Journal of Consulting and Clinical Psychology, 66, 651-654.

Sbrocco, T., Randall, C., Nedegaard, R.C., Stone, J.M., \& Lewis, E.L. (1999). Behavioral choice treatment promotes continuing weight loss: Preliminary results of a cognitive-behavioral decision-based treatment for obesity. Journal of Consulting and Clinical Psychology, 67, 260266.

Schaalma, H.P. (1995). Planned development and evaluation of school-based AIDS/STD education. Thesis Maastricht University.

Schachter, S. (1971). Emotion, obesity and crime. New York:Academic Press.

Schwalberg, M.D., Barlow, D.H., Alger, S.A., \& Howard, L.J. (1992). Comparison of bulimics, obese binge eaters, social phobics, and individuals with panic disorder on comorbidity across DSM-III-R anxiety disorders. Journal of Abnormal Psychology, 101, 675-681.

Seidell, J.C. (1995). Obesity in Europe. Obesity Research, 3, 89s-93s.

Seidell, J.C., \& Rissanen, A.M. (1998). Time trends in the worldwide prevalence of obesity. In G.A. Bray, C. Bouchard, \& W.P.T. James (Eds.), Handbook of Obesity (pp. 79-9I). New York: Marcel Dekker Inc.

Smith, D.E., Marcus, M.D., \& Eldredge, K.L. (1994). Binge eating syndromes:A review of assessment and treatment with an emphasis on clinical application. Behovior Therapy, 25, 635658.

Smith, D.E., Marcus, M.D., \& Kaye, W. (1992). Cognitive-behavioral treatment of obese binge eaters. International Journal of Eating Disorders, 12, 257-262.

Sobal, J., \& Stunkard,A.J. (1989). Socioeconomic status and obesity:A review of the literature. Psychological Bulletin, 105, 260-275.

Specker, S., de Zwaan, M., Raymond, N., \& Mitchell, J. (1994). Psychopathology in subgroups of obese women with and without binge eating disorder. Comprehensive Psychiatry, 35, 185190.

Spitzer, R.L., Devlin, M.J., Walsh, B.T., Hasin, D., Wing, R., Marcus, M., Stunkard, A., Wadden, T., Yanovski, S., Agras, S., Mitchell, J., \& Nonas, C. (1992). Binge eating disorder: A multisite field trial of the diagnostic criteria. International Journal of Eating Disorders, I/, I9|-203.

Spitzer, L., \& Rodin, J. (1981). Human eating behavior: A critical review of studies in normal weight and overweight individuals. Appetite, 2, 293-329.

Spitzer, R.L., Yanovski, S., Wadden, T., Wing, R., Marcus, M.D., Stunkard, A.J., Devlin, M., Mitchell, J., Hasin, D., \& Horne, R.L. (1993). Binge eating disorder: Its further validation in a multisite study. International Journal of Eating Disorders, 13, 137-153.

Spurrell, E.B., Wilfley, D.E., Tanofsky, M.B., \& Brownell, K.D. (1997). Age of onset for binge eating:Are there different pathways to binge eating? International Journal of Eating Disorders, $21,55-65$. 
Stallone, D.D., \& Stunkard, A.J. (1991). The regulation of body weight: Evidence and clinical implications. Annals of Behavioral Medicine, 13, 320-330.

Steen, S.N., Oppliger, R.A., \& Brownell, K.D. (1988). Metabolic effects of repeated weight loss and regain in adolescent wrestlers. Journal of the American Medical Association, 260, 47-50.

Striegel-Moore, R.H., Silberstein, L.R., \& Rodin, J. (1986). Toward an understanding of risk factors for bulimia. American Psychologist, 41, 246-263.

Striegel-Moore, R.H., Wilson, G.T., Wilfley, D.E., Elder, K.A., \& Brownell, K.D. (1998). Binge eating in an obese community sample. International joumal of Eating Disorders, 23, 27-37.

Stuart, R.B. (1967). Behavioral control of overeating. Behoviour Research and Therapy, 5, 357-365. Stunkard, A.J. (1957). The dieting depression: Incidence and clinical characteristics of untoward responses to weight reduction. American Journal of Medicine, 23, 77-86.

Stunkard,A.J. (1958). The management of obesity. New York State Journal of Medicine, 58, 79-87. Stunkard,A.J. (1959). Eating patterns and obesity. Psychiotric Quarterly, 33, 284-295.

Stunkard, A.J., Harris, J.R., Pedersen, N.L., \& McClearn, G.E. (1990). The body-mass index of twins who have been reared apart. New England Journal of Medicine, 322, 1483-1487.

Stunkard, A.J., \& Mendelsohn, M. (1961). Disturbances in body image of some obese persons. Journal of the American Dietetic Association, 38, 328-331.

Stunkard, A.J., Sorensen, T.I.A., Hanis, D., Teasdale, T.W., Chakraborty, R., Schull, W.J., \& Schulsinger, F. (1986). An adoption study of human obesity. New England journal of Medicine, 314, 193-198.

Stunkard,A. J., \& Wadden, T.A. (1992). Psychological aspects of severe obesity. American Journal of Clinical Nutrition, 55, 524S-532S.

Sunday, S.R., Halmi, K.A., Werdann, L., \& Levey, C. (1992). Comparison of body size estimation and eating disorder inventory scores in anorexia and bulimia patients with obese, and restrained and unrestrained controls. International Journal of Eating Disorders, II, I33-149.

Tanco, S., Linden, W., \& Earle,T. (1998). Well-being and morbid obesity in women:A controlled therapy evaluation. International journal of Eating Disorders, 23, 325-339.

Telch, C.F., \& Agras, W.S. (1993). The effects of a very low calorie diet on binge eating. Behavior Therapy, 24, 177-193.

Telch, C.F., \& Agras, W.S. (1994). Obesity, binge eating and psychopathology:Are they related? International Journal of Eating Disorders, 15, 53-61.

Telch, C.F., \& Agras, W.S. (1996). Do emotional states influence binge eating in the obese? International Journal of Eating Disorders, 20, 271-279.

Telch, C.F., Agras, W.S., \& Rossiter, E.M. (1988). Binge eating increases with increasing adiposity. International Journal of Eating Disorders, 7, 115-119.

Telch, C.F., Agras, W.S., Rossiter, E.M., Wilfley, D., \& Kenardy, J. (1990). Group cognitive-behavioral treatment for the nonpurging bulimic: An initial evaluation. Journal of Consulting and Clinical Psychology, 58, 629-635.

Telch, C.F., \& Stice, E. (1998). Psychiatric co-morbidity in women with binge eating disorder: Prevalence rates from a non-treatment-seeking sample. Journal of Consulting and Clinical Psychology, 66, 768-776.

Treasure, J., \& Ward, A. (1997). A practical guide to the use of motivational interviewing in anorexia nervosa. European Eating Disorder Review, 5, $102-114$.

Vitousek, K.B., \& Hollon, S.D. (1990). The investigation of schematic content and processing in eating disorders. Cognitive Therapy and Research, 14, 191-214. 
Vitousek, K., Watson, S., \& Wilson, G.T (1998). Enhancing motivation for change in treatmentresistant eating disorders. Clinical Psychology Review, 18, 391-420.

Wadden, T.A. (1993). Treatment of obesity by moderate and severe caloric restriction: Results of clinical research trials. Annals of Internal Medicine, I/9, 688-693.

Wadden, T.A., \& Bartlett, S.J. (1992). Very low calorie diets:An overview and appraisal. In T.A. Wadden \& T.B. Vanltallie (Eds.), Treatment of the seriously obese patient (pp. 44-79). New York: Guilford Press.

Wadden, T.A., Foster, G.D., \& Letizia, K.A. (1994). One-year behavioral treatment of obesity: Comparison of moderate and severe caloric restriction and the effects of weight maintenance therapy. Journal of Consulting and Clinical Psychology, 62, 165-171.

Wadden, T.A., Sternberg, J.A., Letizia, K.A., Stunkard, A.J., \& Foster, G.D. (1989). Treatment of obesity by very low calorie diet, behavior therapy and their combination: $A$ five year perspective. International Journal of Obesity, 13, 39-46.

Wadden, T.A., \& Stunkard,A.J. (1985). Social and psychological consequences of obesity. Annals of Internal Medicine, 103, $1062-1067$.

Wadden, T.A., Stunkard,A.J., \& Liebschutz, J. (1988). Three-year follow-up of the treatment of obesity by very low calorie diet, behavior therapy, and their combination. Journal of Consulting and Clinical Psychology, 56, 925-928.

Wardle, J. (1995). The assessment of obesity: Theoretical background and practical advice. Behaviour Research and Therapy, 33, $107-117$.

Wells, A.M., Garvin, V., Dohm, F.A., \& Striegel-Moore, R.H. (1997). Telephone-based guided self-help for binge eating disorder: A feasibility study. International Journal of Eating Disorders, 2I, 34I-346.

Westerterp, K.R. (1999). Obesity and physical activity. International Journal of Obesity and Related Metabolic Disorders, 23, 59s-64s.

WHO Expert Committee (1995). Physical Status: The use and interpretation of anthropometry. Technical Report series no. 854. Geneva:WHO.

World Health Organization (1997). Obesity: Preventing and Managing the Global Epidemic. Report on a WHO consultation on obesity. Geneva:WHO.

Wilfley, D.E., Agras, W.S., Telch, C.F., Rossiter, E.M., Schneider, J.A., Cole, A.G., Sifford, L., \& Raeburn, S.D. (1993). Group cognitive-behavioral therapy and group interpersonal psychotherapy for the non-purging bulimic individual: A controlled comparison. Journal of Consulting and Clinical Psychology, 61, 296-305.

Wilfley, D.E., \& Cohen, L.R. (1997). Psychological treatment of bulimia nervosa and binge eating disorder. Psychological Bulletin, 33, 437-454.

Wilfley, D.E., Friedman, M.A., Dounchis, J.Z., Stein, R.I., Welch, R.R., \& Ball, S.A. (2000). Comorbid psychopathology in binge eating disorder: Relation to eating disorder severity at baseline and following treatment. Journal of Consulting and Clinical Psychology, 68, 641649.

Wilfley, D.E., Schwartz, M.B., Spurrell, E.B., \& Fairburn, C.F. (1997). Assessing the specific psychopathology of binge eating disorder patients: Interview or self-report? Behaviour Research and Therapy, 35, 1151-1159.

Wilfley, D.E., Schwartz, M.B., Spurrell, E.B., \& Fairburn, C.G. (2000). Using the Eating Disorder Examination to identify the specific psychopathology of binge eating disorder. International Journal of Eating Disorders, 27, 259-269. 
Williamson, D.A., Cubic, B.A., \& Gleaves, D.H. (1993). Equivalence of body image disturbances in anorexia nervosa and bulimia nervosa. Journal of Abnormal Psychology, 102, $177-180$.

Williamson, D.A., Muller, S.L., Reas, D.L., \& Thaw, J.M. (1999). Cognitive bias in eating disorders: Implications for theory and treatment. Behavior Modification, 23, 556-577.

Wilson, G.T. (1994). Behavioral treatment of obesity: Thirty years and counting. Advances in Behoviour Research and Therapy, 16, 31-75.

Wilson, G.T. (1995). The controversy over dieting. In K.D. Brownell, \& C.G. Fairburn (Eds.) Eating Disorders and Obesity: A comprehensive handbook (pp. 87-95). New York: Guilford Press.

Wilson, G.T. (1996).Acceptance and change in the treatment of eating disorders and obesity. Behovior Therapy, 27, 417-439.

Wilson, G.T. (1996a). Manual-based treatments: The clinical application of research findings. Behaviour Research and Therapy, 34, 295-314.

Wilson, G.T. (1997). Treatment manuals in clinical practice. Behaviour Research and Therapy, 35, 205-210.

Wilson, G.T. (1999). Cognitive behavior therapy for eating disorders: Progress and problems. Behaviour Research and Therapy, 37, s79-s95.

Wilson, G.T., \& Brownell, K.D. (1980). Behavior therapy for obesity: An evaluation of treatment outcome. Advances in Behaviour Research and Therapy, 3, 49-86.

Wilson, G.T., Nonas, C.A., \& Rosenblum, G.D. (1993). Assessment of binge eating in obese patients. International Journal of Eating Disorders, 13, 25-33.

Wilson, G.T.,Vitousek, K.M., \& Loeb, K.L. (2000). Stepped care treatment for eating disorders. Journal of Consulting and Clinical Psychology, 68, 564-572.

Wing, R.R. (1992). Weight cycling in humans: A review of the literature. Annals of Behavioral Medicine, 14, $113-119$.

Wooley, S.C., \& Garner, D.M. (199I). Obesity treatment:The high cost of false hope. Journal of the American Dietetic Association, 91, 1248-1251.

Yanovski, S.Z. (1993). Binge eating disorder: Current knowledge and future directions. Obesity Research, 1, 306-324.

Yanovski, S.Z., Gormally, J.F., Leser, M.S., Gwirtsman, H.E., \& Yanovski, J.A. (1994). Binge eating disorder affects outcome of comprehensive very-low-calorie diet. Obesity Research, 2, 205-212.

Yanovski, S.Z., Leet, M., Yanovski, J.A., Flood, M., Gold, P.W., Kissileff, H.R., \& Walsh, B.T. (1992). Food selection and intake of obese women with binge eating disorder. American Journal of Clinical Nutrition, 56, 975-980.

Yanovski, S.Z., Nelson, J.E., Dubbert, B.K., \& Spitzer, R.L. (1993). Association of binge eating disorder and psychiatric comorbidity in obese subjects. American Journal of Psychiatry, I50, 1472-1479.

Yanovski, S.Z., \& Sebring, N.G. (1994). Recorded food intake of obese women with binge eating disorder before and after weight loss. International Journal of Eating Disorders, 15, 135-150.

Young, J.E. (1990). Cognitive therapy for personality disorders: A schema-focused approach. Sarasota, FL: Professional Resource Exchange. 


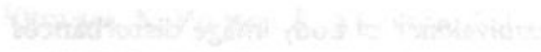

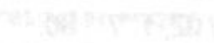




\section{SUMMARY}

Obesity is a prevalent problem. In the Netherlands the prevalence of overweight (BMI $>27$ ) is approximately $24 \%$. In Western society, being overweight or obese is generally viewed as a more serious problem for females. Females worry more than men about their shape and weight, are more critical about their bodies, and want to weigh less. The desire for thinness has led to an increase of diet pills, websites, weight loss programs, magazine articles and books about the newest and quickest ways to lose weight. This thesis focuses on the effectiveness of a non-dieting behavioral and a cognitive therapy for obese women with obesity and binge eating disorder.

Chapter 1 gives a brief historical framework for the development of attitudes about obesity, as well as a description of the definition and prevalence of obesity. A subgroup of obese persons reporting problems with binge eating is introduced. Persons with binge eating disorder (BED) eat large amounts of food in a short period of time. This eating is accompanied by perceived loss of control and subsequent distress. Unlike bulimia nervosa patients, persons with BED do not use any compensatory behaviors following a binge. Differences in eating disorder symptomatology and comorbidity between obese binge eaters and obese non-binge eaters is given. The traditional behavioral therapy programs focusing on quick and large weight loss for obese persons are critically reviewed. The first, and most important criticism against traditional behavioral therapy is that they are generally successful in the short-term, but are often not very effective in maintaining weight loss in the long-term. Studies have shown that almost all participants returned to their baseline weights or eventually weighed even more than they did at the start of treatment ('jo-jo'effect). In addition, traditional behavioral therapy might have certain risks. Weight cycling might increase mortality and morbidity, decreases the resting metabolic rate and increases the proportion of body fat. Finally, strict dieting might result in pathological changes in cognitions and affect and might lead to binge eating.

Chapter 2 introduces a non-dieting cognitive-behavioral model for obese binge eaters and obese non-binge eaters. This model is based on theories and ideas about disturbed eating behavior and dysfunctional cognitions. Cognitive-behavioral therapy aims at improving psychological well-being, restructuring dysfunctional cognitions regarding body weight and shape, and restoring normal and healthy eating patterns, rather than at weight loss per se. An update is given of studies examining the effects of cognitive-behavioral therapy for obese binge eaters and obese non-binge eaters.

Chapter 3 outlines the cognitive and behavioral therapy protocols and introduces the empirical studies of this thesis. The primary aim of this thesis is to examine which part of the cognitive-behavioral therapy is effective in obesity and binge eating disorder, the cognitive part or the non-dieting behavioral part? So far, these two components have never been examined separately in obese persons. The cognitive therapy was expected to produce greater changes in psychological well-being (depression and self-esteem) and dysfunctional cogni- 
tions, although in the short term weight loss was expected to be greater in the behavioral therapy.

Chapter 4 reports the frequency and content of several cognitions in obese women with and without BED. A structured clinical interview was used to detect differences in cognitions. The majority of obese binge eaters mentioned negative self-schemas that could be characterized as negative generalizations about the self combined with shape, weight and eating concerns. For example 'If I weigh more than $100 \mathrm{~kg}$, than I am good for nothing'. The majority of obese non-binge eaters mentioned shape, weight and eating concerns that were not combined with negative generalizations about the self. Participants with negative self-schemas, irrespective of binge category, were more depressed and had lower self-esteem than the other participants. Finally, with respect to the content analyses of negative self-schemas, it was found that both groups most often mentioned themes such as rejection, unworthiness, and lack of willpower. However, obese non-binge eaters mentioned more self-schemas regarding lack of willpower, whereas obese binge eaters were more preoccupied with rejection and unworthiness.

Chapter 5 evaluates the short-term effectiveness of a cognitive and a behavioral therapy. Seventy-four obese participants, 37 binge eaters, and 37 non-binge eaters were randomly assigned to one of the two treatment conditions taking binge status into account. Both treatments took place in groups and all groups met for 15 weekly sessions of 150 minutes each. Participants in the cognitive therapy learned to change dysfunctional cognitions and participants in the behavioral therapy learned a regular and healthy eating pattern. Ten participants (13.5\%) dropped out of treatment. Both treatments were effective in reducing a broad spectrum of complaints. At posttreatment the cognitive therapy was significantly more effective than the behavioral therapy in reducing concerns about shape, weight, and eating, and in improving self-esteem. At 6-month follow-up the cognitive therapy remained superior at improving shape, weight, and eating

134 concerns. The behavioral therapy was more effective in reducing weight, but at 6-month follow-up participants in the behavioral therapy regained weight. Binge eating was reduced in both treatments equally effectively, but at 6-month participants who received cognitive therapy were more abstinent from binge eating. Obese binge eaters benefited more from treatment than obese non-binge eaters and this may be due to floor effects in the latter group.

Chapter 6 evaluates the long-term effectiveness of both treatments and the role of weight changes on psychological well-being. Analyses on the completers' sample $(\mathrm{N}=60)$ revealed that both treatments had a markedly positive and remaining impact on shape, weight, and eating concerns, binge eating, selfesteem and depression at 1-year follow-up. Cognitive therapy was not superior to behavioral therapy on most outcome measures. This might be due to selective drop-out. At 1-year follow-up, four women refused to take part in the assessment. All four women had received behavioral therapy and three of them were at baseline identified as binge eaters. At 1-year follow-up, these obese binge eaters reported objective binge episodes and the obese non-binge eater 
reported a chaotic eating pattern and severe depressive symptoms. Analyses including non-responders $(\mathrm{N}=64)$ showed that the cognitive therapy was superior to the behavioral therapy in changing binge eating and shape, weight, and eating concerns. The results for weight-loss were disappointing. Between pretreatment and 1-year follow-up participants in the behavioral therapy lost $3.0 \mathrm{~kg}$, while participants in the cognitive therapy lost $0.3 \mathrm{~kg}$. However, participants who gained weight were as successful in changing psychological wellbeing as participants who lost weight.

Chapter 7 reports a study which aims to examine pretreatment patient characteristics as predictors of 1-year outcome after treatment for obesity. In addition, this study examines the central prediction for the cognitive view of obesity, namely participants' shape and weight concerns at posttreatment predict outcome at 1-year follow-up. It was found that less shape and weight concerns and a high motivation for therapy at pretreatment were predictive of less severe eating disorder pathology at 1-year follow-up. Binge episodes at pretreatment and a family history of obesity were predictive of a higher BMI at follow-up. Furthermore, it was shown that less shape and weight concerns directly after treatment predicted less severe eating pathology at 1-year follow-up.

Chapter 8 provides a summary and discussion of the main empirical findings, followed by a review of methodological strengths and limitations. Finally, suggestions for practice are given. A stepped-care strategy is promoted. In this strategy simple and cheap approaches (e.g. self-help) might be undertaken as the first line of intervention, since a number of obese persons will respond. Those who do not benefit, receive more intensive treatment and move on to the next 'step'. The protocols of the interventions may serve as a promising tool for specialized therapist in (mental) health settings to improve psychological well-being, dysfunctional cognitions, and a disturbed eating pattern in obese persons with and those without binge eating problems. 


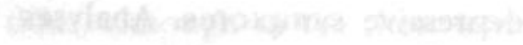

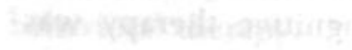

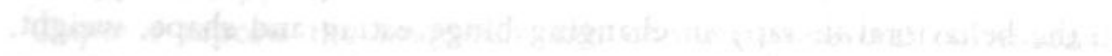

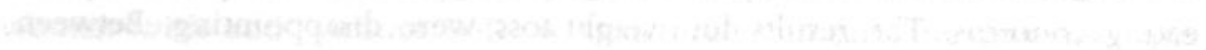

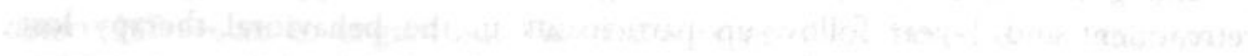

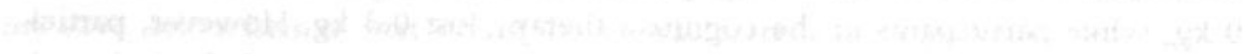

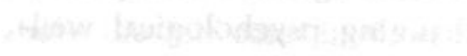

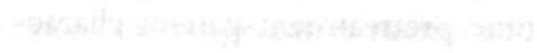

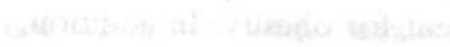




\section{SAMENVATTING}

Obesitas, een andere naam voor ernstig overgewicht, is een veelvoorkomend probleem. In Nederland heeft ongeveer $25 \%$ van de bevolking last van overgewicht (BMI>27). In onze westerse maatschappij wordt vooral door vrouwen het hebben van overgewicht problematisch gevonden. Vrouwen maken zich meer dan mannen zorgen over hun figuur en gewicht, zijn kritischer ten aanzien van hun lichaam, en willen vaak minder wegen. De wens om slank te zijn heeft tot een enorme toename geleid van dieetpillen, cursussen, websites, tijdschriftartikelen, en boeken over manieren om snel gewicht te verliezen. In dit proefschrift ligt de nadruk op de effectiviteit van een niet op lijnen gerichte gedragstherapeutische en cognitieve therapie voor obese vrouwen met en zonder eetbuistoornis.

In hoofdstuk 1 wordt een korte historische schets over de houding ten aanzien van obesitas beschreven. Daarnaast wordt de definitie en de prevalentie van obesitas besproken. Een subgroep van obese mensen, namelijk mensen die problemen rapporteren met eetbuien, wordt geïntroduceerd. Deze personen met een eetbuistoornis ('binge eating disorder') eten een grote hoeveelheid voedsel in een korte periode, ervaren controleverlies, en na afloop van de eetbui voelt men zich ellendig. In tegenstelling tot mensen met bulimia nervosa, maken mensen met een eetbuistoornis geen gebruik van gewichtscontrole maatregelen. Verschillen in eetpathologie en co-morbiditeit tussen obese mensen met en zonder eetbuistoornis worden besproken. De huidige afslankprogramma's voor obese mensen worden kritisch onder de loep genomen. Het belangrijkste argument tegen deze programma's is dat uitsluitend op korte-termijn gewichtsverlies wordt gerealiseerd, en dat ze op de lange-termijn niet effectief zijn. Studies hebben aangetoond dat bijna iedereen weer terugvalt en evenveel of zelfs meer weegt dan voor het programma (jojo-effect). Verder zijn er mogelijk risico's verbonden aan afslankprogramma's. Het zogenoemde jojo-effect kan zorgen voor gezondheidsproblemen, een verlaging van het rustmetabolisme en een verhoging van het vet percentage. Ten slotte kan streng lijnen mogelijk leiden tot zowel pathologische veranderingen in cognities en stemming, maar ook tot eetbuien.

In hoofdstuk 2 wordt een cognitief-gedragstherapeutisch model voor obese mensen met en zonder eetbuistoornis geïntroduceerd. Het model is gebaseerd op reeds bestaande theorieën en ideeën over een verstoord eetpatroon en disfunctionele cognities. De cognitieve-gedragstherapie die voortvloeit uit dit model heeft tot doel het bevorderen van het psychisch welbevinden, herstructureren van disfunctionele cognities over lichaam en gewicht, en het herstellen van een normaal en gezond eetpatroon, in plaats van primair de aandacht te richten op gewichtsverlies. Een overzicht wordt gegeven van studies waarin het effect van cognitieve-gedragstherapie voor obese mensen met en zonder eetbuistoornis is onderzocht.

Hoofdstuk 3 geeft een gedetailleerde beschrijving van het cognitieve en het gedragstherapeutische protocol en introduceert de empirische studies. Een van de belangrijkste onderzoeksvragen in dit proefschrift is om te kijken welk deel 
van de cognitieve-gedragstherapie het meest effectief is voor obese vrouwen met en zonder eetbuistoornis, de cognitieve component of de gedragstherapeutische component? Tot op heden zijn deze componenten nog niet afzonderlijk onderzocht bij obese vrouwen. Van de cognitieve therapie werd verwacht dat deze tot een groter psychologisch welbevinden en minder disfunctionele cognities zou leiden. Echter op de korte termijn werd verwacht dat het gewichtsverlies groter zou zijn in de gedragstherapie.

In hoofdstuk 4 wordt verslag gedaan van het onderzoek naar de frequentie en de inhoud van de diverse cognities bij obese vrouwen met en zonder eetbui-stoornis. Er werd gebruik gemaakt van een gestructureerd klinisch interview. De meerderheid van de obese vrouwen met een eetbuistoornis noemden negatieve zelfschema's die gekarakteriseerd kunnen worden als negatieve uitspraken over zichzelf gecombineerd met zich zorgen maken over gewicht, lichaam en eten. Bijvoorbeeld 'Als ik meer dan 100 kilogram weeg, ben ik niets waard'. De meerderheid van de obese vrouwen zonder eetbuistoornis maakten zich zorgen over gewicht, lichaam en eten zonder dat ze dit combineerden met negatieve uitspraken over zichzelf. Onafhankelijk van het feit of mensen wel of niet een eetbuistoornis hadden, voelden de deelneemsters met veel negatieve zelf-schema's zich depressiever en hadden ze een lagere zelfwaardering. Ten slotte werd de inhoud van de negatieve zelf-schema's onderzocht. Er werd gevonden dat beide groepen het vaakst thema's noemden als zich afgewezen voelen, zich minderwaardig voelen, en een gebrek aan wilskracht. Vrouwen zonder eetbui-stoornis noemden vaker zelf-schema's die betrekking hadden op gebrek aan wilskracht, terwijl mensen met een eetbuistoornis zich meer afgewezen en minderwaardig voelden.

Hoofdstuk 5 beschrijft de korte-termijn effectiviteit van de cognitieve therapie en de gedragstherapie. Vierenzeventig deelneemsters met overgewicht, waarvan 37 met eetbuistoornis en 37 zonder eetbuistoornis, werden at random verdeeld over de twee behandelcondities. Beide behandelingen vonden plaats in groepen. De groepen kwamen 15 keer bijeen gedurende 150 minuten. Deelneemsters aan de 138 cognitieve therapie leerden om disfunctionele cognities te veranderen en deelneemsters aan de gedragstherapie leerden hoe ze gezond en regelmatig konden eten. Tien deelneemsters (13.5\%) waren uitgevallen. Beide behande-lingen bleken effectief in het verminderen van diverse klachten. Direct na behandeling was cognitieve therapie significant beter dan gedragstherapie in het reduceren van zorgen omtrent gewicht, figuur, en eten, en in het verhogen van de zelfwaardering. Tijdens de follow-up (6 maanden) bleef de cognitieve therapie effectiever in het reduceren van zorgen omtrent gewicht, figuur en eten. De gedragstherapie bleek effectiever in het reduceren van het gewicht, echter tijdens de follow-up (6 maanden) bleken de deelneemsters weer in gewicht toegenomen. De frequentie van eetbuien nam direct na beide behandelingen af. Tijdens de follow-up (6 maanden) waren meer deelneemsters aan de cognitieve therapie abstinent, dat wil zeggen dat zij helemaal geen eetbuien meer hadden. Vrouwen met een eetbuistoornis profiteerden meer van behandeling dan vrouwen zonder eetbuistoornis. Dat kan het gevolg zijn van het feit dat vrouwen zonder eetbuistoornis al lagere scores tijdens de voormeting hadden en niet meer verder konden verbeteren. 
Hoofdstuk 6 beschrijft de lange-termijn effectiviteit ( 1 jaar na behandeling) van beide behandelingen en de rol van gewichtsverlies ten aanzien van psychologisch welbevinden. Allereerst werden analyses verricht met de groep obese vrouwen die de behandeling voltooiden en tevens tijdens de follow-up metingen aanwezig waren $(\mathrm{N}=60)$. Hieruit bleek dat tijdens de follow-up ( 1 jaar) beide behandelingen nog steeds bleven resulteren in een reductie van zorgen omtrent figuur, gewicht en eten, eetbuien, en depressie en in een verhoging van de zelfwaardering. Cognitieve therapie bleek niet langer effectiever dan gedragstherapie. Dit kan het gevolg zijn van selectieve uitval. Tijdens de follow-up (1 jaar) weigerden vier vrouwen om de vragenlijsten in te vullen. Deze vier vrouwen namen allen deel aan gedragstherapie en drie van hen hadden tijdens de voormeting een eetbuistoornis. Tijdens de follow-up rapporteerden drie vrouwen last te hebben van frequente eetbuien en de vrouw zonder eetbui-stoornis vermelde een chaotisch eetpatroon en depressieve symptomen. Analyses die verricht werden inclusief deze vier 'non-responders' $(\mathrm{N}=64)$ lieten vervolgens een superieur effect zien van de cognitieve therapie in vergelijking met gedragstherapie ten aanzien van het reduceren van eetbuien en zorgen omtrent figuur, gewicht en eten. De resultaten voor gewichtsverlies waren ook één jaar na behandeling teleurstellend. Deelneemsters aan gedragstherapie verloren in totaal 3 kilogram en deelneemsters aan cognitieve therapie 0.3 kilogram. Echter deelneemsters die waren toegenomen in gewicht, bleken even succesvol in het verbeteren van psychologisch welbevinden als deelneemsters die waren afge-vallen.

Hoofdstuk 7 doet verslag van een studie waarin gezocht wordt naar predictoren (diverse karakteristieken van de deelneemsters op de voormeting) voor het resultaat van de behandeling tijdens de follow-up (1 jaar). Verder wordt er onderzocht of de zorgen over lichaam en gewicht tijdens de nameting het resultaat van behandeling op de lange-termijn voorspellen. De resultaten lieten zien dat minder zorgen over lichaam en gewicht en een hoge motivatie tijdens de voormeting predictoren waren van minder ernstige eetpathologie tijdens de follow-up. Het hebben van eetbuien en familieleden met obesitas bleken predictoren voor een hogere BMI tijdens de follow-up. Verder bleek dat hoe minder zorgen men maakte over lichaam en gewicht direct na de behandeling hoe minder ernstig de eetpathologie was tijdens de follow-up.

Hoofdstuk 8 bevat een samenvatting en een discussie van de belangrijkste empirische bevindingen, gevolgd door een overzicht van de methodologische sterke en zwakke punten van het onderzoek. Tevens worden er suggesties voor de praktijk gegeven. Een stapsgewijs zorgmodel wordt voorgesteld. In dit model worden simpele en goedkope behandelingen (bijvoorbeeld zelf-hulp groepen) voorgesteld als primaire interventie. Een aantal obese mensen zal mogelijk met deze benadering geholpen zijn. Diegene die niet verbeteren, krijgen een intensievere behandeling en schuiven door naar de volgende 'stap'. De protocollen van de behandelingen kunnen dienen als middel voor gespecialiseerde therapeuten binnen de (geestelijke) gezondheidszorg om verbeteringen te bewerkstelligen in psychologisch welbevinden, disfunctionele cognities, en een verstoord eetpatroon in obese mensen met en zonder eetbuistoornis. 


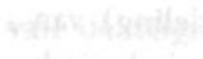

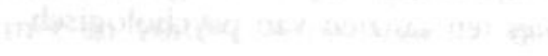

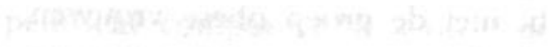

4

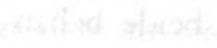




\section{DANKWOORD}

Poeh keek naar de stok in zijn handen.

'Ik vond hem gewoon daarginds,' zei hij.

'Ik dacht dat ik hem mooi kon gebruiken om Roe te helpen

en toen heb ik hem gewoon opgeraapt.'

'Poeh,' zei Janneman Robinson plechtig.

'De Expeditie heeft haar doel bereikt! Jij hebt de Noordpool gevonden!'

Uit: Tyerman Williams (1996). Poeh en de filosofen, p. 164.

Het proefschrift is af. Ik wil de volgende mensen bedanken die, ieder op eigen wijze, een bijdrage hebben geleverd aan het voltooien van deze expeditie:

\section{(Co)-promotoren}

Harm Hospers, Anita Jansen

De obese vrouwen die hebben deelgenomen aan het onderzoek

Onderzoeksassistenten en studenten

Inge Drost, Olga Haveman, Aimée Jansen, Mirjam Notermans, Wieke Pasman, Christianne Verwegen, Maaike de Vries

\section{Therapeuten}

Christel Achterberg, Maaike Breuer, Jolanda Coolen, Hermien Elgersma, Gerdy

Konings, Roos Rensen, Monique Wijers

Kamergenoten en collega's van Gezondheidsvoorlichting/poli-eetstoornissen en de leden van de intervisiegroep therapie evaluatie onderzoek

Met name Gerjo Kok, Trijntje Völlink, Elke Bollen, Marcella Gijzen, Hanneke Oomkes, Franz Wojciechowski

Vormgeving proefschrift

Nora Oosting (grafische vormgeving), Josee Driesssen (ontwerp omslag)

Paranymfen

Marie-Anne Haenen, Idith Lavy

and last but certainly not least ...

Arjan, Jaron en mijn ouders 



\section{CURRICULUMVITAE}

Helga Nauta werd op 18 januari 1964 in Amstelveen geboren. Na het behalen van het VWO diploma aan het Casimir Lyceum in Amstelveen, begon zij aan de opleiding fysiotherapie in Amsterdam. Vanaf 1985 studeerde zij Gezondheidswetenschappen aan de Universiteit Maastricht. In 1991 behaalde zij het doctoraal diploma van de afstudeerrichting Geestelijke Gezondheidkunde. Zij had tevens blokken Gezondheidsvoorlichting gevolgd. In aansluiting daarop was ze twee jaar als toegevoegd docent aangesteld bij het Departement Medische, Klinische en Experimentele Psychologie van de Universiteit Maastricht. Daarna werkte zij van september 1993 tot september 1998 als Assistent In Opleiding (AIO) bij de capaciteitsgroep Gezondheidsvoorlichting van de Universiteit Maastricht. Deze aanstelling mondde uit in het schrijven van dit proefschrift. Zij volgde in deze periode tevens de VGT-opleiding tot gedragstherapeut en startte in 1998 bij de RINO Zuid-Nederland de insteekroute voor psychotherapeut. In 1997 behaalde zij het gewoon lidmaatschap van de Nederlandse Vereniging voor Gedragstherapie en in 1999 werd zij geregistreerd als GZ-psycholoog. Sinds januari 1999 heeft zij een vaste aanstelling bij de poli-eetstoornissen van het Academisch Ziekenhuis Maastricht en verricht zij vooral diagnostiek en behandeling bij mensen met anorexia nervosa. In het voorjaar van 2002 heeft zij de opleiding voor psychotherapeut afgerond en zal zij zich als psychotherapeut laten registreren. 
号 



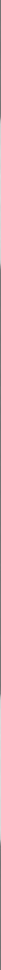

